\title{
La subtribu Plucheinae (Inuleae, Asteraceae) en México: taxonomía, diversidad y distribución
}

\section{The subtribe Plucheinae (Inuleae, Asteraceae) in Mexico: taxonomy, diversity, and distribution}

\section{Acta Botanica Mexicana}

\author{
Rosario Redonda-Martínez,2
}

\section{Resumen:}

Antecedentes y Objetivos: La subtribu Plucheinae es un grupo escasamente representado en México y por ello no debería presentar problemas taxonómicos. Sin embargo, esto no es así, pues se han confundido taxones con hábitos de crecimiento contrastantes como Pluchea carolinensis y $P$. odorata. Además, se ha reportado la presencia de especies de otros países debido a identificación errónea, de ahí la necesidad de generar un tratamiento taxonómico actualizado. El objetivo de este trabajo es presentar una sinopsis taxonómica actualizada de las Plucheinae mexicanas, incluyendo claves de identificación y descripciones de géneros y especies.

Métodos: Se hizo una revisión de trabajos florístico-taxonómicos de las tribus Inuleae y Plucheeae realizados previamente en México, para generar un listado actualizado de especies mexicanas que posteriormente se cotejó con ejemplares herborizados depositados en las colecciones científicas mexicanas ENCB, FCME, IEB y MEXU, además de herbarios en línea de los Estados Unidos de América.

Resultados clave: En México, Pluchea es el género más diverso de la subtribu Plucheinae y de distribución más amplia, agrupa ocho especies y dos categorías infraespecíficas, se encuentra en 27 estados. Oaxaca y Veracruz concentran el mayor número de especies, y la menor diversidad está en Aguascalientes, Ciudad de México y Durango, donde se registra únicamente un taxón. Las Plucheinae ocurren en elevaciones desde el nivel del mar hasta 3100 m s.n.m. y tienen afinidad por ambientes con humedad elevada.

Conclusiones: El agrupamiento de las cabezuelas y el tipo de indumento en los filarios han sido caracteres útiles para diferenciar taxones mexicanos del complejo P. foetida; sin embargo, es necesario realizar estudios anatómicos y micromorfológicos en este complejo para corroborar su separación.

Palabras clave: Compositae, Epaltes, nomenclatura, Pluchea, Pseudoconyza, Pterocaulon.

\section{Abstract:}

Background and Aims: The subtribe Plucheinae is a poorly represented group in Mexico and therefore should not present taxonomic problems. However, this is not the case, since taxa with contrasting growth habits such as Pluchea carolinensis and $P$. odorata have been confused, and the presence of species from other countries has been reported due to erroneous identification; hence, the need to generate an updated taxonomic treatment. The aim of this work is to present an updated taxonomic study of the Mexican Plucheinae, including identification keys and descriptions of genera and species.

Methods: A revision of floristic-taxonomic works of the tribes Inuleae and Plucheeae was carried out to generate a list of species present in Mexico, which was compared with the collections of Mexican herbaria ENCB, FCME, IEB and MEXU, in addition to online herbaria from the United States of America.

Key results: In Mexico, Pluchea is the genus of the subtribe Plucheinae with the widest diversity and distribution, grouping eight species and two infraspecific categories, being found in 27 states. Oaxaca and Veracruz concentrate the highest number of species, and the least diversity is found in Aguascalientes, Mexico City and Durango, where only one taxon is registered. The Plucheinae occur in elevations that range from sea level to $3100 \mathrm{~m}$ a.s.I. They have an affinity for environments with high humidity.

Conclusions: The grouping of heads and the indumentum on the phyllaries have been useful to differentiate Mexican taxa from the $P$. foetida complex; however, it is necessary to carry out anatomical and micromorphological studies in this complex to corroborate their separation.

Key words: Compositae, Epaltes, nomenclature, Pluchea, Pseudoconyza, Pterocaulon.

${ }^{1}$ Instituto de Ecología A.C., Red de Diversidad Biológica del Occidente Mexicano, Centro Regional del Bajío. Av. Lázaro Cárdenas 253, 61600, Pátzcuaro, Michoacán, México.

22Autor para la correspondencia: r.redonda.martinez@ gmail.com
Recibido: 20 de abril de 2020.

Revisado: 13 de mayo de 2020.

Aceptado por Marie-Stéphanie Samain: 16 de junio de 2020.

Publicado Primero en línea: 1 de julio de 2020

Publicado: Acta Botanica Mexicana 127(2020).
Citar como: Redonda-Martínez, R. 2020. La subtribu Plucheinae (Inuleae, Asteraceae) en México: taxonomía, diversidad y distribución. Acta Botanica Mexicana 127: e1718. DOI: 10.21829/ abm127.2020.1718 cc) (7) (8) Este es un artículo de acceso abierto c) Atribución-No Comercial (CC BY-NC 4.0 Internacional). 


\section{Introducción}

Asteraceae es una de las familias más diversas de Angiospermas al agrupar 25,000-35,000 especies, que representan 10\% de su total (Mandel et al., 2019); incluye 13 subfamilias y 44 tribus (Panero y Crozier, 2016). La subfamilia Asteroideae concentra el mayor número de tribus y especies, en particular en la supertribu Helianthodae. Ésta cuenta con más de 3000 taxones en Norteamérica, pero en otras regiones su presencia es mínima (Panero y Crozier, 2016). En América del Norte existen tribus cuya riqueza es baja, como en el caso de Inuleae. Esta tribu fue descrita por Cassini (1819), quien la reconoció por presentar anteras caudadas y dos líneas estigmáticas separadas en las ramas del estilo. Sus miembros se caracterizan por presentar follaje aromático, cabezuelas heterógamas, disciformes, a veces radiadas, flores periféricas femeninas, corolas filiformes o liguladas, flores del disco bisexuales o funcionalmente masculinas; anteras caudadas, estilos con ramas pilosas y vilano de cerdas capilares (Anderberg y Eldenäs, 2007; Anderberg, 2009).

La circunscripción taxonómica de Inuleae ha cambiado a través del tiempo. Bentham (1873) mantuvo la definición de Cassini, aunque agregó géneros como Blumea DC. y Laggera Sch. Bip. ex Benth. \& Hook f., descritos para incluir en ellos especies de Conyza Less., con características distintas a las de este género, entre las que destacan la presencia de filarios conspicuamente graduados, vilano con pocas cerdas capilares, anteras caudadas y ramas del estilo con líneas estigmáticas apicales. También agrupó en Inuleae a Epaltes Cass. y Pluchea Cass., clasificados previamente en Vernonieae (Cassini, 1817, 1818a, b), por compartir distribución en trópicos y subtrópicos de ambos hemisferios.

A finales del siglo XX ocurrieron cambios en la circunscripción de Inuleae. Merxmüller et al. (1977) la dividieron en las subtribus Athrixiinae, Gnaphaliinae e Inulinae. En esta última reconocieron tres grupos: Buphthalminae, Inulinae y Plucheinae, que presentaban ramas del estilo con bandas estigmáticas confluentes, granos de polen con báculas y capa basal no entrelazada. En la subtribu Gnaphaliinae agruparon especies con ramas del estilo con bandas estigmáticas separadas (tipo Senecio L.), granos de polen sin báculas y capa basal entrelazada; mientras que en la subtribu Athrixiinae incluyeron taxones con características intermedias entre Gnaphaliinae e Inulinae, al presentar ramas del estilo tipo Senecio y granos de polen con capa basal no entrelazada.

Los primeros estudios filogenéticos realizados en Inuleae trajeron consigo cambios en su clasificación. Al encontrarse que no era monofilética, Anderberg (1989) reconoció tres grupos: Inuleae s.s., Gnaphalieae y Plucheeae y elevó los dos últimos a rango de tribu. En un trabajo posterior, se halló evidencia adicional para reconocer a Gnaphalieae como una tribu diferente de Inuleae, así como la posición basal de ésta y Plucheeae en la subfamilia Asteroideae (Kim y Jansen, 1995). Sin embargo, análisis recientes (Panero y Crozier, 2016; Mandel et al., 2019), basados en caracteres moleculares, ubican a Inuleae en posición intermedia en la subfamilia, pero basal en la supertribu Helianthodae (Panero y Crozier, 2016; Mandel et al., 2019). Estudios moleculares realizados en Inuleae han identificado dos linajes: InuleaePlucheinae e Inuleae-Inulinae (Eldenäs et al., 1999; Anderberg et al., 2005), que corresponden a dos subtribus. La subtribu Inulinae presenta estilos con ramas agudas y cristales de oxalato de calcio en la epidermis de la cipsela, mientras que Plucheinae tiene estilos con ramas obtusas y carece de cristales en la cipsela (Anderberg, 2009).

A nivel mundial, la tribu Inuleae incluye 62 géneros y alrededor de 700 especies (Anderberg, 2009). En México está representada únicamente por la subtribu Plucheinae, con cuatro géneros y 12 especies, de las cuales dos son endémicas (Villaseñor, 2018). Dada la escasa diversidad de este grupo en territorio nacional, no debería presentar problemas taxonómicos o de circunscripción. Sin embargo, esto no es así; muestra de ello es la confusión de taxones con hábitos de crecimiento contrastantes como Pluchea carolinensis (Jacq.) G. Don y P. odorata (L.) Cass. (Goodfrey, 1952), así como la identificación errónea de algunas especies que ha dado lugar a reportar su presencia en el país, como ha ocurrido con P. foetida (L.) DC. (Villaseñor y Villarreal, 2006; Nesom, 2020). Además, el género Pseudoconyza Cuatrec. ha sido considerado sinónimo de 
Blumea DC. (Badillo, 1974; Medina-Lemos y VillaseñorRíos, 2010; Redonda-Martínez, 2016). Por lo anterior, un estudio taxonómico actualizado de la subtribu en el país resultará útil para las personas que realizan estudios florísticos y requieren identificar el material botánico que recolectan, sobre todo si procede de zonas distintas a las que comprenden las floras regionales que han publicado tratamientos taxonómicos de este grupo (McVaugh, 1984; Medina-Lemos y Villaseñor-Ríos, 2010; Redonda-Martínez, 2016; Pruski, 2018).

El objetivo de este trabajo es presentar una sinopsis taxonómica actualizada de las Plucheinae mexicanas, la cual incluye claves de identificación, descripciones de géneros y especies, información de fenología, distribución por estado y tipos de vegetación.

\section{Materiales y Métodos}

Se hizo una revisión de trabajos florístico-taxonómicos de Asteraceae y, en particular, de las tribus Plucheeae e Inuleae realizados en México (McVaugh, 1984; Villaseñor y Villarreal, 2006; Medina-Lemos y Villaseñor-Ríos, 2010; Redonda-Martínez, 2016; Pruski, 2018), para generar un listado preliminar de especies. Éste se cotejó con ejemplares herborizados. Con el propósito de contar con una representación lo más completa posible, se consultaron las colecciones: MEXU (Herbario Nacional de México, Instituto de Biología, Universidad Nacional Autónoma de México), ENCB (Escuela Nacional de Ciencias Biológicas, Instituto Politécnico Nacional) y FCME (Facultad de Ciencias, Universidad Nacional Autónoma de México), donde se encuentran especímenes del centro y sur del país, particularmente San Luis Potosí, Estado de México, Guerrero, Oaxaca, Chiapas y Veracruz, entidades con una riqueza considerable de especies de Asteraceae (Villaseñor, 2018). Los ejemplares representativos del occidente se consultaron en IEB (Instituto de Ecología, A.C., Centro Regional del Bajío).

Para complementar la revisión de especímenes del norte de México escasamente representados en los herbarios antes citados, se consultaron las colecciones en línea de BCMEX (Universidad Autónoma de Baja California) (SEINet, 2018) y algunas de los Estados Uni- dos de América: ARIZ (University of Arizona) (SEINet, 2018), ASU (Arizona State University) (SEINet, 2018), COLO (University of Colorado Museum) (SEINet, 2018), DES (Desert Botanical Garden) (SEINet, 2018), F (Field Museum of Natural History) (BCFM, 2018), MO (Missouri Botanical Garden) (TROPICOS, 2018), NY (The New York Botanical Garden) (NYBG, 2018), UCR (University of California, Riverside) (SEINet, 2018) y US (Smithsonian Institution) (BCS, 2018). Los acrónimos de las colecciones consultadas se abrevian de acuerdo con Thiers (2019). Por último, en el portal electrónico Global Plants (JSTOR, 2018), se revisaron los ejemplares tipo de las especies incluidas en el listado, así como sus respectivos sinónimos.

Con base en esta revisión se generó una base de datos para recopilar toda la información disponible en las etiquetas de colecta (distribución por estado, vegetación, rango altitudinal, localidades de recolecta, hábito, tamaño de la planta y fenología), la cual, sumada a la revisión morfológica de los especímenes, se utilizó para elaborar claves de identificación y descripciones de cada taxón. En el caso particular de los tipos de vegetación citados en las etiquetas y con el propósito de homogenizarlos, se utilizaron las propuestas de Miranda y Hernández X. (1963) e INEGI (2017); cada uno se define con base en su fisonomía, derivada de las formas de vida de las especies dominantes (Miranda y Hernández X., 1963). Para los ecotonos se usó el concepto de Holland y Risser (1991), considerándolos transiciones entre comunidades vegetales diferentes a lo largo de gradientes ambientales.

Con el propósito de ilustrar caracteres morfológicos importantes de la subtribu, se tomaron fotografías de cabezuelas y flores con un microscopio estereoscópico (Leica EZ4HD, Heerbrugg, Suiza) con cámara integrada, con una resolución de 600 dpi. Las imágenes digitalizadas de ejemplares herborizados depositados en MEXU se obtuvieron con un escáner HerbScan (Epson Expression Model 10000XL, Graphic Arts, Suwa, Nagano, Japón) con una resolución de $600 \mathrm{dpi}$. Para georreferenciar las localidades de recolecta se utilizó Google Earth Pro (Google Earth, 2018) y los mapas de distribución se elaboraron con ArcMap 10.2 (ESRI, 2013). 


\section{Resultados}

\section{Diversidad y distribución}

Los cuatro géneros de la subtribu Plucheinae presentes en México son Epaltes, Pseudoconyza, Pterocaulon Elliot y Pluchea. Los tres primeros incluyen una especie cada uno, mientras que Pluchea agrupa ocho y dos variedades. Éste también es el género con distribución más amplia al encontrarse en 27 de los 32 estados del país, seguido de Pseudoconyza que ocurre en 14; Epaltes solo está en seis y Pterocaulon, en dos. Los estados de Oaxaca y Veracruz albergan el mayor número de especies con siete y dos variedades, solo que en Veracruz existen los cuatro géneros. En Aguascalientes, Ciudad de México y Durango se registra únicamente un taxón; mientras que en Tlaxcala y Zacatecas están ausentes.

Las Plucheinae se encuentran en elevaciones desde el nivel del mar hasta 3100 m s.n.m. Tienen afinidad por zonas con humedad elevada, ya que el mayor número de taxones (11 especies y dos variedades) crece en vegetación hidrófila, seguida de manglares (cuatro). También son tolerantes al disturbio, pues en vegetación secundaria se encontraron siete especies y las dos variedades.

Pluchea carolinensis, P. salicifolia (Mill.) S.F. Blake y $P$. odorata presentan la mayor distribución geográfica y por tipo de vegetación, al encontrarse en 27,22 y 17 estados, así como en 15, ocho y ocho tipos de vegetación, además de ecotonos. Pluchea mexicana (Goodfrey) G.L. Nesom y P. parvifolia (A. Gray) Goodfrey tienen distribución restringida, al encontrarse solo en un estado, uno y cinco tipos de vegetación, respectivamente. Por otra parte, P. mexicana, P. yucatanensis G.L. Nesom y Pterocaulon virgatum (L.) DC. únicamente se han registrado en tres localidades de colecta, con lo cual pueden catalogarse como taxones raros, con un nivel de rareza tipo IV (Rabinowitz et al., 1986). Parece que son especies con distribución geográfica restringida, posiblemente con alta especificidad de hábitat y quizás el tamaño de sus poblaciones sea reducido. Los ejemplares de $P$. mexicana se colectaron a fines del siglo XIX y principios del XX, mientras que los de $P$. yucatanensis y $P$. virgatum datan de las décadas de 1970 y 1980, a pesar de que en las regiones donde se encontraron han realizado estudios florísticos en años recientes (Flora de Veracruz, 1978+; CICY, 2010+; De-Nova et al., 2018).

\section{Tratamiento taxonómico}

Inuleae Cass., J. Phys. Chim. Hist. Nat. Arts 88: 193. 1819. TIPO: Inula L.

Hierbas o arbustos, a veces árboles, generalmente aromáticos; tallos erectos o decumbentes, ramas tomentosas, pilosas o glandulares; hojas alternas, ocasionalmente opuestas, simples, enteras o pinnatisectas; cabezuelas heterógamas, rara vez homógamas, con calículo; inflorescencias cimosas, corimbiformes, paniculiformes, en ocasiones cabezuelas solitarias; involucro cilíndrico, campanulado o hemisférico, a veces turbinado, filarios dispuestos en varias series, graduadas o subiguales, libres, receptáculo plano o convexo, desnudo o paleáceo; flores periféricas femeninas y fértiles, con corolas filiformes, radiadas o tubulares, rosadas, moradas, amarillas u ocroleucas; por lo general en mayor número que las flores del disco; flores del disco bisexuales o funcionalmente masculinas, con corolas tubulares o infundibuliformes, amarillas, rosadas o moradas, rara vez blanquecinas, anteras con apéndices apicales ovados, lanceolados o deltoides; ramas del estilo filiformes, agudas u obtusas, papilosas por debajo de la bifurcación; cipselas elipsoides, cilíndricas, claviformes o fusiformes; costilladas, anguladas o estriadas; pilosas, glandulares, o glabras, con vilano uni o multiseriado, con cerdas capilares barbeladas, a veces lisas, o escamas alargadas, libres, rara vez formando una corona anular, en ocasiones ausente (Fig. 1).

Clave para identificar géneros de la subtribu Plucheinae presentes en México

1a. Inflorescencias espiciformes simples Pterocaulon Elliott

1b. Inflorescencias corimbiformes, a veces cimosas .......... 2

2a. Hierbas anuales, tallos alados; cipselas sin vilano ......... Epaltes Cass.

2b. Hierbas perennes, o arbustos, con tallos sin alas, rara vez alados; cipselas con vilano 3 

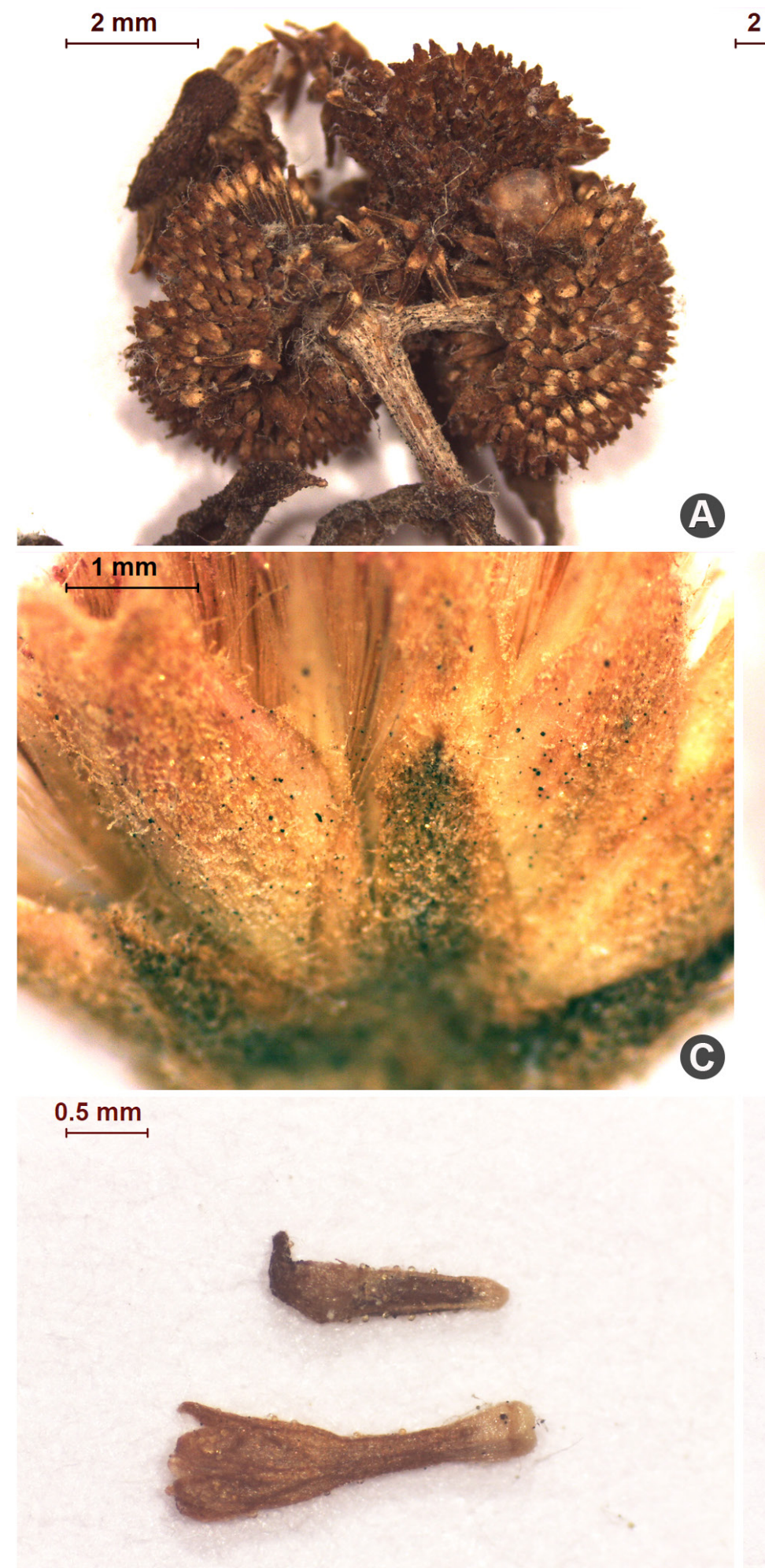

$2 \mathrm{~mm}$

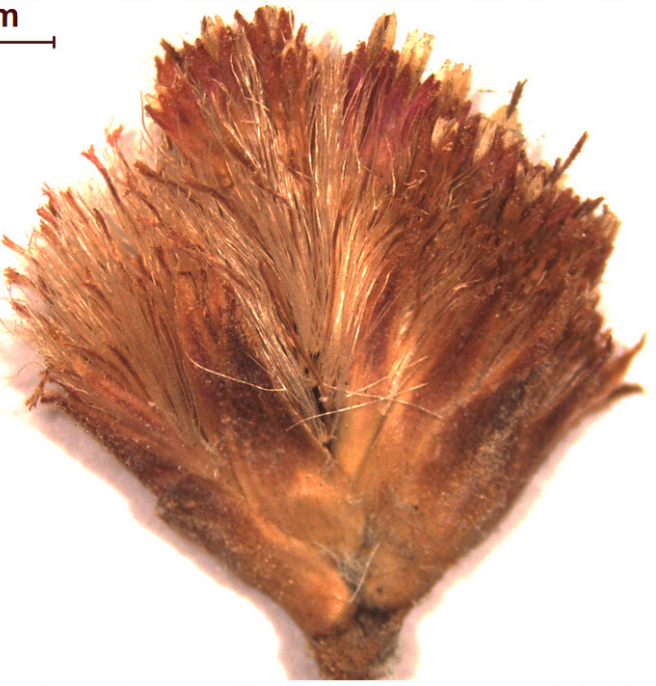

B
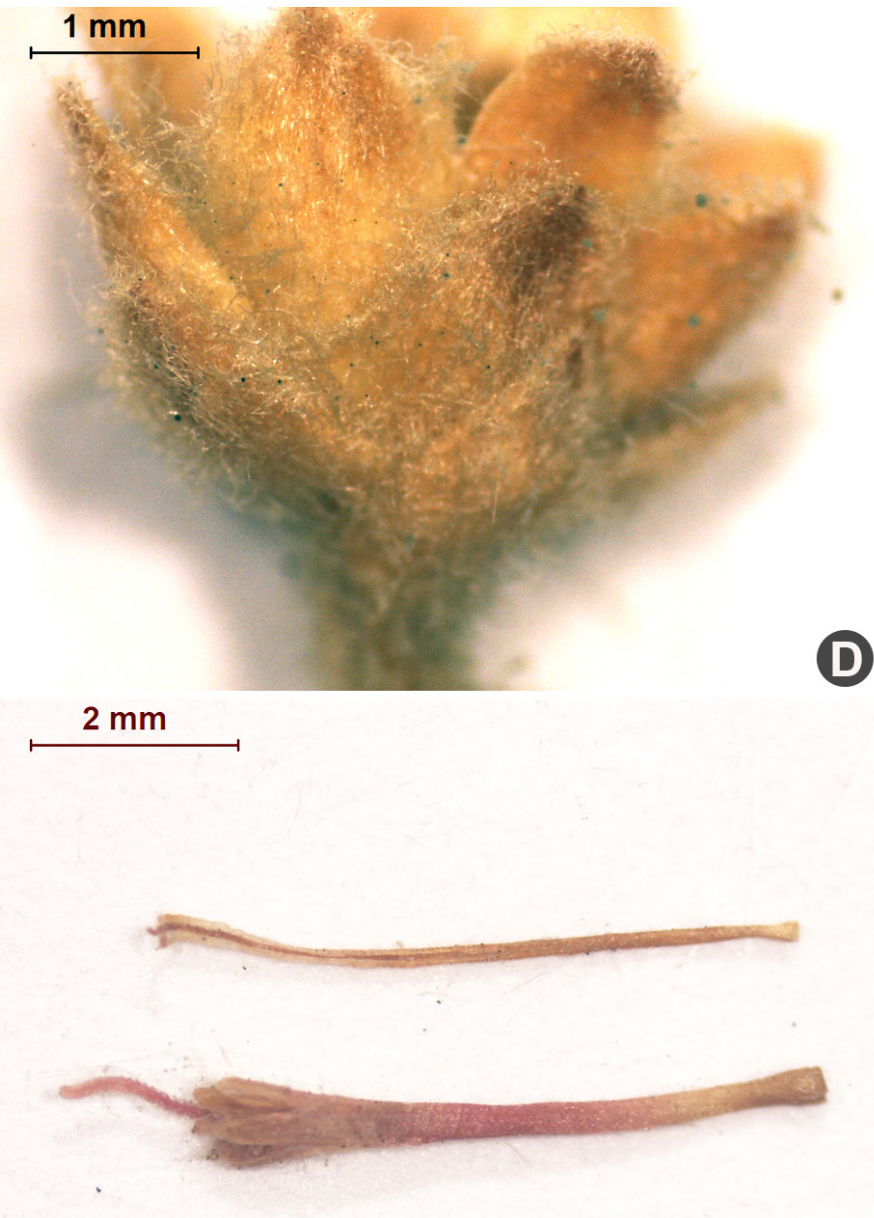

E

Figura 1: Características morfológicas de algunas Plucheinae mexicanas. A. cabezuelas de Epaltes mexicana Less.; B. cabezuela de Pluchea baccharis (Mill.) Pruski; C. indumento piloso y glandular en el involucro de Pluchea odorata (L.) Cass.; D. indumento seríceo en Pluchea sericea (Nutt.) Coville; E. flores de Epaltes mexicana Less., arriba flor periférica tubular, femenina y abajo, flor tubular central funcionalmente masculina, ambas con glándulas; F. flores de Pluchea sericea (Nutt.) Coville, arriba flor periférica filiforme, femenina y abajo, flor central tubular, bisexual. 
3a. Hojas pinnatisectas, flores del disco hermafroditas, vilano de cerdas capilares lisas

Pseudoconyza Cuatrec.

3b. Hojas enteras, flores del disco funcionalmente masculinas, vilano de cerdas capilares barbeladas

Pluchea Cass.

Epaltes Cass., Bull. Sci. Soc. Philom. Paris 1818: 138. 1818.

TIPO: Epaltes divaricata (L.) Cass.

= Sphaeromorphaea DC., Prodr. 6: 140. 1837.

= Ethuliopsis F. Muell., Frag. 2(16): 154. 1861.

= Pachytelia Steetz, Naturw. Reise Mossambique 2: 453. 1864.

= Gynaphanes Steetz, Naturw. Reise Mossambique 2: 457. 1864.

इCentipeda sect. Sphaeromorphaea (DC.) C.B. Clarke, Compos. Ind. 151. 1876, nom. illeg. superfl.

E Epalthes sect. Ethuliopsis F. Muell., Frag. 10(86): 100. 1877.

$=$ Erigerodes L. ex Kuntze, Revis. Gen. PI. 1: 335. 1891.

= Poilania Gagnep., Bull. Soc. Bot. France 71: 56. 1924.

Hierbas anuales o perennes, a veces sufrútices; taIlos alados, puberulentos o glabros; hojas alternas, sésiles, lanceoladas, elípticas u ovadas, margen entero, serrado o dentado; cabezuelas dispuestas en inflorescencias cimosas o corimbiformes; involucro hemisférico; filarios graduados, 3 seriados, receptáculo aplanado o convexo, rara vez cóncavo en el centro, glabro, desnudo; flores periféricas numerosas, femeninas, corolas tubulares, rosadas; ramas del estilo triangulares y aplanadas en el ápice, pilosas; flores del disco funcionalmente masculinas, a veces bisexuales, corolas tubulares, rosadas; anteras blanquecinas, apéndices apicales ovados, base caudada; cipselas claviformes, 4 costilladas, pardas o negras, glandulares, vilano ausente.

Epaltes incluye 14 especies, está relacionado con Cylindrocline Cass., Doellia Sch. Bip., Karelinia Less. y Laggera Sch. Bip. ex Benth. \& Hook. f. Se distribuye en trópicos y subtrópicos de ambos hemisferios (Anderberg,
2009). Epaltes se diferencia de otros géneros de Inuleae por el vilano ausente o reducido (Anderberg y Eldenäs, 2007). En México solo está representado por una especie, Epaltes mexicana Less., la cual extiende su distribución a Centroamérica.

Epaltes mexicana Less., Linnaea 5(1): 147. 1830. TIPO: MÉXICO. Veracruz, In humidis prope Veracruz, VII.1828, C. J. W. Schiede 322 (holotipo: HAL-0098214!, isotipos: $\mathrm{P}-01816122$ !, dibujo y fragmento $\mathrm{GH}-00006583$ !).

इ Pachytelia mexicana (Less.) Steetz, Naturw. Reise Mossambique 2: 454.1864.

$\equiv$ Erigerodes mexicanus (Less.) Kuntze, Revis. Gen. PI. 1: 335. 1891.

Hierbas anuales, 0.3-1.1 m de alto; ramas tomentulosas; hojas con láminas lanceolado-elípticas, rara vez ovadoelípticas, 3-8.2 × 0.8-3 cm, ápice agudo, a veces obtusomucronato, base decurrente, margen dentado, pilosoglandulares en ambas superficies; inflorescencias cimosas con 3-20 cabezuelas, pedúnculos individuales 4.8-11.6 $\mathrm{mm}$ de largo, tomentosos; involucro 1.2-2.7 $\times$ 2.6-6.5 $\mathrm{mm}$; filarios (20-)24-26, imbricados, elípticos o elípticolanceolados, ápice agudo-mucronato, tomentulosos y glandulares; flores periféricas 398-486(-500), corolas 0.7-0.9 mm de largo, estilo $0.6-0.8 \mathrm{~mm}$ de largo, cipselas 0.8-0.96 mm de largo, glandulares; flores del disco 42-50, corolas $1.2-2 \mathrm{~mm}$ de largo, rosadas, a veces amarillentas; anteras 0.8-1 mm de largo (Figs. 1A, E, 2).

Comentarios taxonómicos: en Global Plants (JSTOR, 2018) se cita al ejemplar del herbario $P$ colectado por C. J. W. Schiede con el número 96, como sintipo de $E$. mexicana. Sin embargo, el holotipo que se encuentra en HAL (0098214!), corresponde a un espécimen de Schiede con el número 322; al igual que el dibujo y fragmento del tipo en GH. En ambos casos, Schiede colocó una nota que reza: Obr. 96. Probablemente esta sea la razón de la confusión con los números de la colección tipo; pues tanto el holotipo como el "sintipo" se colectaron en Veracruz en julio de 1828. 


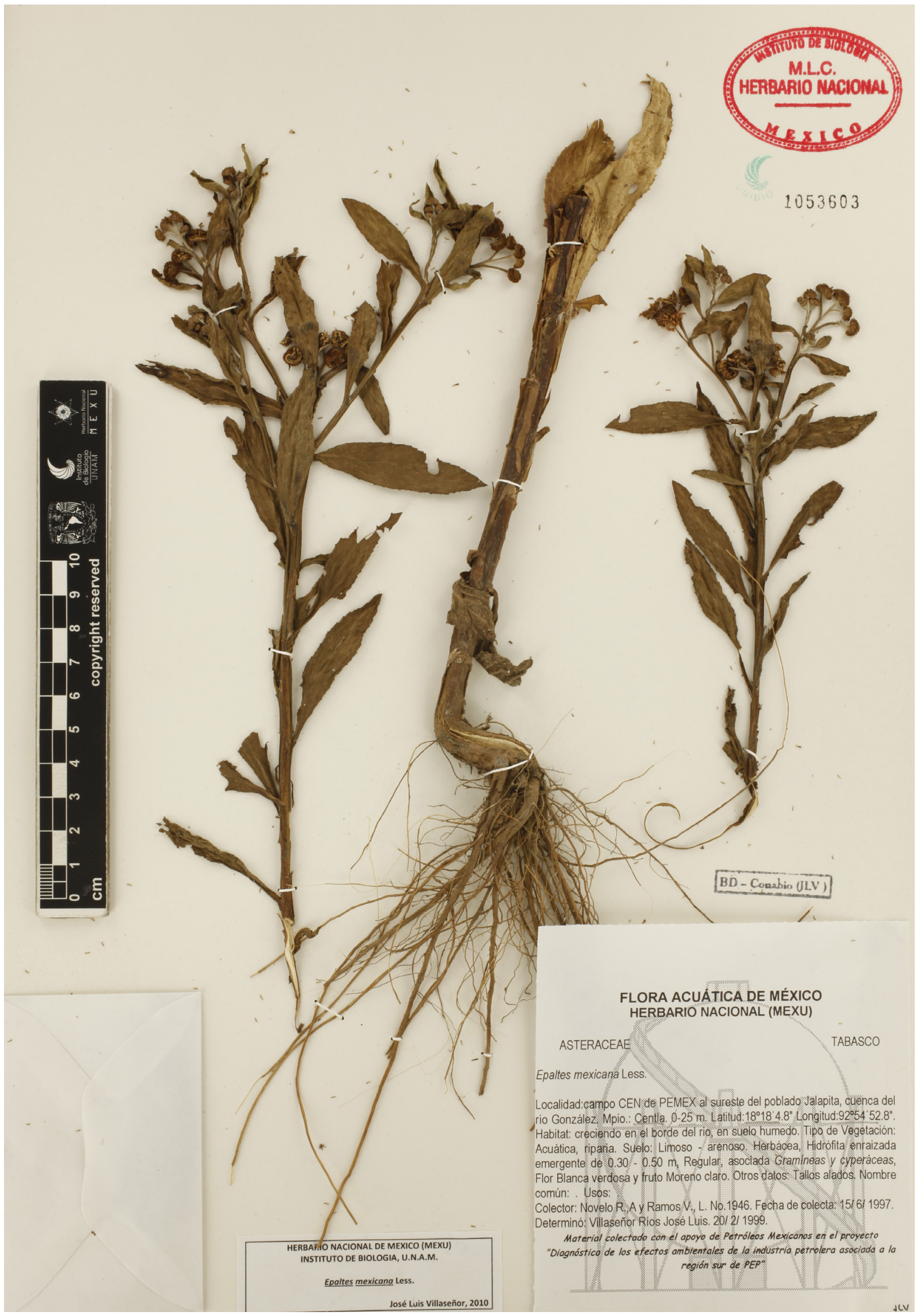

Figura 2: Ejemplar herborizado de Epaltes mexicana Less. 
Distribución: en México, Epaltes mexicana se encuentra en Campeche, Chiapas, Oaxaca, Puebla, Tabasco y Veracruz, en elevaciones de 0-700 m s.n.m. (Fig. 3).

Hábitat: manglares, selvas altas perennifolias, vegetación de dunas costeras, halófila, hidrófila y secundaria, en suelos arenosos, arcillosos o limosos, ocasionalmente alcalinos.

Fenología: florece y fructifica de marzo a noviembre.

Ejemplares examinados: MÉXICO. Campeche, municipio Champotón, alrededor de la aguada Paraíso, 8.VIII.1985, C. Chan 3785 (MEXU, MO). Chiapas, municipio Catazajá, orilla de la Laguna Catazajá, 17.IV.1987, A. Ortega 208 (MEXU). Municipio Ocosingo, $3 \mathrm{~km}$ al E de Pico de Oro, camino a Benemérito de las Américas, 18.IV.1986, E. Martínez S. 18409 (MEXU, MO). Oaxaca, municipio San Juan Guichicovi, San Juan Guichicovi, centro del poblado, III.1986, A. Nereyda y M. Heinrich 36 (MEXU). Municipio San Juan Lalana, Jalahui, VII.1986, M. Heinrich 26 (MEXU). Municipio Santa María Chimalapa, $1.5 \mathrm{~km}$ al SSO de Santa María Chimalapa, orilla del Río Negro, 23.VI.1990, S. Acosta 1910 (MEXU); along small path in central Santa María Chimalapa, 4.VII.2014, M. S. Geck 163 (MEXU). Municipio Santiago Comaltepec, Puerto Eligio, 28.VI.1975, J. Rzedowski 33401 (ENCB, MEXU). Municipio Santo Domingo Petapa, Santo Domingo Petapa, 21.X.1992, B. Frei 151 (MEXU). Puebla, municipio Francisco Z. Mena, Cerro Tablón, 7.II.1986, S. Vargas 168 (MEXU). Municipio Hueytamalco, rancho Las Margaritas, 7.VI.1976, W. López-Forment 140 (MEXU). Tabasco, municipio Balancán, La Palma, 1.VI.1939, E. Matuda 3315 (MEXU). Municipio Cárdenas, cerca del muelle, en la laguna El Yucateco, 11.VI.1997, A.M. Hanan et al. 948 (MEXU); Cinco Presidentes, área situada entre Villa Benito Juárez y Sánchez Magallanes (porción N), 12.VI.1996, A. Novelo y L. Ramos 1572 (MEXU). Municipio Centla, Campo CEN de PEMEX, pantano seco a orilla del Río Tular, A. $M$. Hanan 1015 (MEXU); Arroyo San Pedrito, 4.VI.1998, M. A. Guadarrama et al. 6448 (MEXU); rancho El Esoro, ejido El Faisán, 10.VI.1998, M. A. Guadarrama et al. 6713 (MEXU); Campo CEN de PEMEX, SE del poblado Jalapita, cuenca del Río González, 15.VI.1997, A. Novelo y L. Ramos 1946 (IEB,
MEXU, MO); Lagunas Pajaral I y II, aproximadamente $20 \mathrm{~km}$ al $\mathrm{S}$ de Frontera, laguna que desemboca al Río San Pedrito, 8.VII.1998, A. Novelo y L. Ramos 2713 (MEXU, MO). Municipio Centro, Rancho Tumbulushal, carretera Villahermosa Teapa km 20, 10.V.1993, P. Alegría 6 (MEXU); Río Carrizal, islote formado al interior del Río Carrizal, cerca de la estación de Macayo, 8.VI.1996, A. Novelo y L. Ramos 1491 (MEXU). Municipio Huimanguillo, rumbo a Mecatepec Ocuapan, 9 $\mathrm{km}$ al S de Huimanguillo, km 2 rumbo a Francisco Rueda, 16.I.1979, R. Margado y C. P. Cowan 1791 (MEXU); loc. cit., 16.I.1979, A. Martínez y C. P. Cowan 1894 (MEXU); Río Tonalá, entre Agua Dulce y Las Choapas, 16.VI.1997, A. Novelo y L. Ramos 1867 (MEXU, MO). Municipio Jonuta, Laguna Cantemó, frente al ejido San José, aproximadamente 40 $\mathrm{km}$ al SE de Frontera, rumbo a Jonuta, 16.V.1998, A. Novelo y L. Ramos 2556 (MEXU). Municipio Paraíso, La Unión, orilla derecha del Río Tupilquillo, 25.VII.1967, R. Guzmán s.n. (MEXU). Veracruz, municipio Alto Lucero de Gutiérrez Barrios, $3 \mathrm{~km} \mathrm{~N}$ de Salinas, carretera Veracruz - Alvarado, 18.VII.1967, L. I. Nevling y A. Gómez 82 (MEXU). Municipio Alvarado, carretera Veracruz - Alvarado, km 19, 5.VII.1977, J. J. Fay y C. Hernández 808 (MEXU). Municipio Catemaco, $500 \mathrm{~m}$ de la Playa de la Basura, Laguna de Sontecomapan, 28.V.1969, W. Boege 1263 (MEXU); Zapopan de Cabañas, Los Tuxtlas, 1953, H. Bravo 79 (MEXU). Municipio Chacaltianguis, Chacaltianguis, Cosamaloapan, 20.V.1969, G. Martínez 1904 (MEXU). Municipio Coatzacoalcos, 0.4 km al E de los quemadores de PEMEX, en el área de inventario, 4.IV.2003, C. H. Ramos y E. Martínez S. 2323 (MEXU); 3.17 $\mathrm{km}$ al SW del hotel de solteros, sobre los ductos de PEMEX que dividen la congregación Nuevo Mundo, 9.V.2003, C. H. Ramos y E. Martínez S. 2347 (MEXU). Municipio Cosamaloapan de Carpio, Lower Papaloapan Basin, cerros de Los Domingos, Mound A (back levee of the Papaloapan), archaeological site, 19.VII.1974, Large 38 (ASU). Municipio Jesús Carranza, Santa Lucrecia, VIII.1936, E. Matuda 769 (MEXU); Río Vista, Río Coatzacoalcos; 1.5 km de Jesús Carranza, 9.VIII.1971, L. I. Nevling y A. Gómez 2554 (MEXU). Municipio Las Choapas $2 \mathrm{~km}$ de Las Choapas, detrás del rancho El Roble, 18.IX.1974, Brigada de vegetación acuática 57 (MEXU). Municipio Minatitlán, 9 km de Minatitlán, rumbo a Coatzacoalcos, 19.VI.1973, A. D. Orozco 214 (MEXU); pantano Santa Alejandrina, 9.VI.2004, M. A. Teno- 


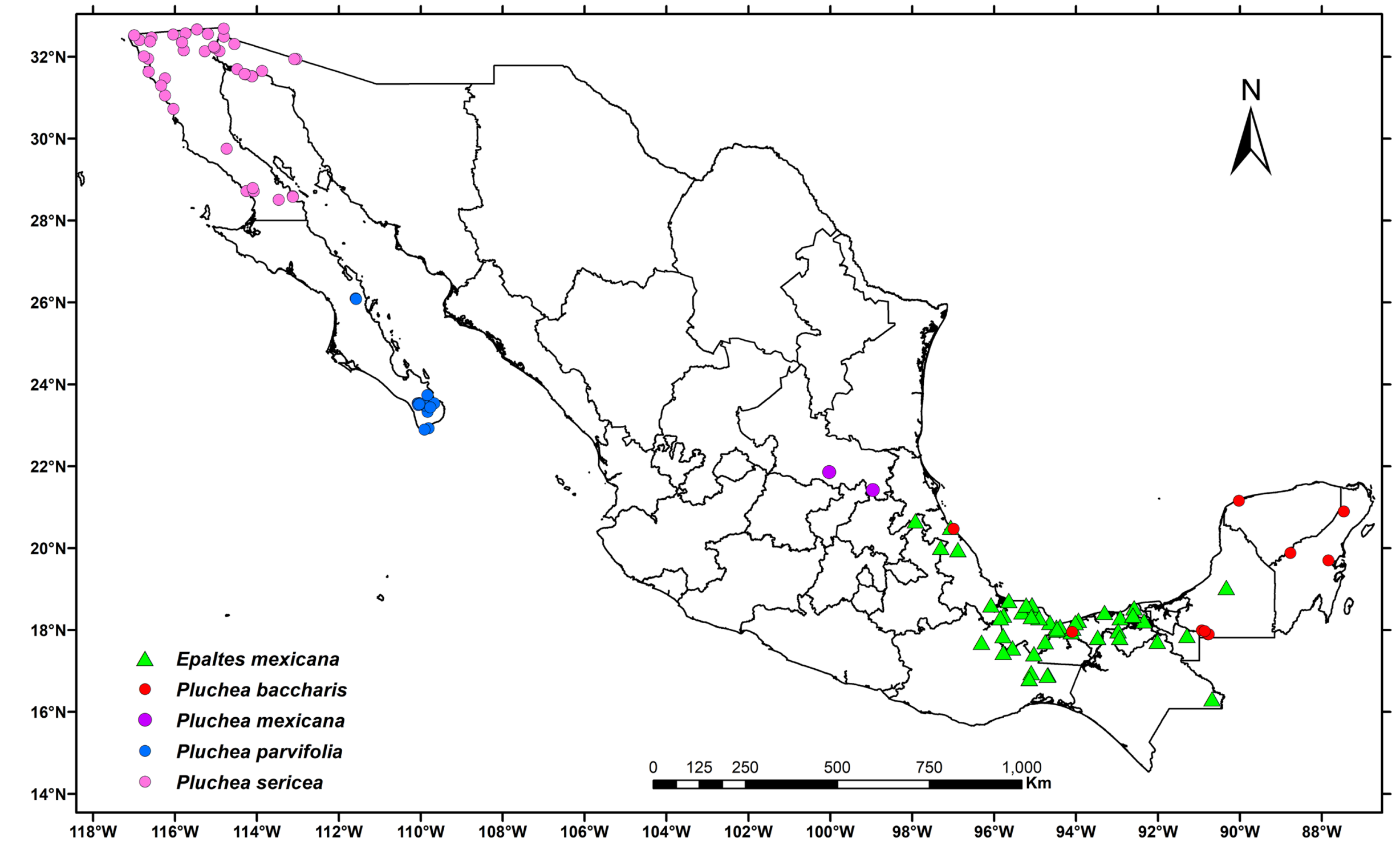

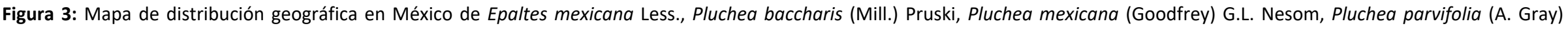
Goodfrey y Pluchea sericea (Nutt.) Coville. 
rio 13 (MEXU). Municipio Misantla, Santa Cruz de Hidalgo, carretera Misantla - Martínez de la Torre, 28.IV.1976, A. Hernández 398 (NY). Municipio Nanchital de Lázaro Cárdenas del Río, 6.78 km al SW de Nanchital, 11.IX.2011, J. Calónico y B. Gómez 27707 (MEXU). Municipio Pajapan, Laguna del Ostión, 28.III.1980, J. Dorantes y L. Gutiérrez 58 (MEXU). Municipio Playa Vicente, $12 \mathrm{~km}$ antes de Playa Vicente, cerca de La Victoria (3 km), 11.IV.1969, L. I. Nevling y A. Gómez 670 (MEXU); $3 \mathrm{mi} \mathrm{W}$ of Yoqope, then $6 \mathrm{mi}$ NW to first bridge, 16.II.1975, M. Williams y K. Engelen s.n. (MEXU). Municipio San Andrés Tuxtla, Balzapote, 19.V.1981, Anónimo 255 (MEXU); loc. cit., 8.VIII.1979, Anónimo 724 (MEXU); loc. cit., 18.VI.1970, G. Martínez 2293 (MEXU); region of San Andrés Tuxtla, along river draining Laguna de Catemaco, 12.VIII.1953, R. L. Dressler y Q. Jones 52 (MEXU, NY); along a small creek beside route 180 at km 130, a few miles of San Andrés Tuxtla, 22.V.1979, N. A. Harriman 15755 (MEXU); Playa de Balzapote, 27.V.2001, A. Rincón et al. 2489 (MEXU). Municipio Santiago Tuxtla, $3 \mathrm{~km}$ al SW de Santiago Tuxtla, por la carretera a Villa Isla, a lo largo del Río Tepango, en el puente del mismo nombre, 31.V.1985, J. L. Villaseñor y W. W. Thomas 837 (MEXU, NY). Municipio Tecolutla, Estero de La Silveña, cerca de Tecolutla, 26.IV.1965, J. Rzedowski 19988 (ENCB, MEXU). Municipio Tequixtepec, San Lorenzo Tenochtitlán, 18.XI.1995, C. H. Ramos y E. Martínez S. $1077 b$ (MEXU).

Pluchea Cass., Bull. Sci. Soc. Philom. Paris 1817: 31. 1817. TIPO: Conyza marilandica Michx.

$=$ Monarrheus Cass., Bull. Sci. Soc. Philom. Paris 1817: 31. 1817, nom. nud.

= Gynema Raf., Fl. Ludov. 63. 1817.

三 Gymnostyles Raf., Fl. Ludov. 150. 1817, nom. illeg. hom., non Juss. 1804.

三 Stylimnus Raf., J. Phys. Chim. Hist. Nat. Arts 89: 100. 1819, nom. illeg. superfl.

= Conyza subgen. Leptogyne Elliot, Sketch Bot. S. Carolina 2: 322.1824.

= Tecmarsis DC., Prodr. 5: 375. 1836.

= Bertelothia DC., Prodr. 5: 375. 1836.

三 Pluchea sect. Stylimnus (Raf.) DC., Prodr. 5: 540. 1836, nom. illeg. superfl.
= Eyrea F. Muell., Linnaea 25(4): 403. 1852, nom. illeg. hom., non Champ. ex Benth. 1851.

= Tessaria sect. Phalacrocline A. Gray, Smithsonian Contr. Knowl. 3(5): 102. 1852.

= Spiropodium F. Muell., Fragm. 1(2): 33. 1858.

झ Pluchea sect. Eyrea Benth., Fl. Austral. 3: 527. 1866.

三Pluchea sect. Berthelotia (DC.) A. Gray, Proc. Amer. Acad.

Arts 17: 212. 1882, nom. illeg. superfl.

三 Eremohylema A. Nelson, Univ. Wyoming Publ. Sci. Bot. 1(3): 54. 1924, nom. illeg. superfl., nombre propuesto por Nelson (1924) para remplazar a Berthelotia.

Arbustos o hierbas perennes, rara vez arborescentes; tallos alados o sin alas, pilosos o tomentosos, rara vez glabrescentes con la edad; hojas alternas, corto o largo pecioladas, ocasionalmente sésiles, ovadas, elípticas o linear-lanceoladas; inflorescencias cimosas, corimbiformes o paniculiformes; involucro turbinado, campanulado o hemisférico, filarios 3-4 seriados, graduados, receptáculo convexo, glabro, desnudo; flores periféricas numerosas, femeninas, corolas filiformes, rosadas o moradas; flores del disco en menor número que las periféricas, funcionalmente masculinas, corolas tubulares, rosadas o moradas; ramas del estilo teretes, redondeadas y papilosas en el ápice; anteras blanquecinas, ápice lanceolado, base sagitada; estilo con ramas obtusas en el ápice, papilosas; cipselas fusiformes, 6-8 costilladas, pardas o negras, pilosas y glandulares, vilano de cerdas capilares barbeladas.

Pluchea agrupa alrededor de 80 especies con distribución mundial (Anderberg, 2009), de las cuales ocho y dos variedades se encuentran en México. Las relaciones filogenéticas del género no están resueltas, aunque forma parte del núcleo Plucheoide junto con Coleocoma F. Muell., Cylindrocline, Doellia, Epaltes, Karelinia, Laggera, Peuchel-loeschea O. Hoffm., Porphyrostemma Grant ex Benth. \& Hook. f., Pseudoconyza, Sphaeranthus L., Streptoglossa Steetz ex F. Muell. y Tessaria Ruiz \& Pav. (Anderberg, 2009).

Clave para identificar especies de Pluchea presentes en México

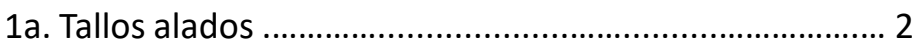

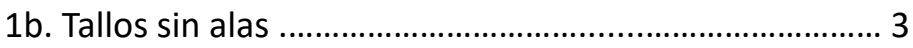


2a. Filarios linear-lanceolados, 3-4 seriados, involucro $\leq 6.5$ $\mathrm{mm}$ de largo P. salicifolia (Mill.) S.F. Blake

2b. Filarios lanceolados, 6-7 seriados, involucro $\geq 6.4 \mathrm{~mm}$ de largo P. parvifolia (A. Gray) Godfrey

3a. Arbustos, ramas pilosas, tomentosas o seríceas, sin glándulas 4

3b. Hierbas anuales o perennes, ramas pilosas o glandulares, a veces glabrescentes 5

4a. Hojas lanceoladas o linear-lanceoladas, tomentosas o seríceas en el envés; filarios 3-4 seriados P. sericea (Nutt.) Coville

4b. Hojas elípticas u ovado-elípticas, pilosas en el envés; filarios 5-6 seriados ......... P. carolinensis (Jacq.) G. Don

5 a. Hierbas anuales; hojas con peciolos $\geq 9 \mathrm{~mm}$ de largo, indumento piloso en ambas superficies; flores periféricas $\geq 340$ P. odorata (L.) Cass.

5b. Hierbas perennes; hojas sésiles; indumento glandular en una o ambas superficies, flores periféricas $\leq 200$... 6

6 a. Hojas con indumento glandular; filarios 24-28; flores periféricas $188-200$ P. yucatanensis G.L. Nesom

6 b. Hojas con indumento piloso y glandular; filarios 28-36; flores periféricas 110-130 7

7a. Hojas con ápice agudo; inflorescencias con 12 o más cabezuelas; vilano de las flores periféricas $\geq 18$ cerdas; vilano de las flores del disco $\leq 20$ cerdas

P. mexicana (Godfrey) G.L. Nesom

7b. Hojas con ápice apiculado; inflorescencia con 10 o menos cabezuelas; vilano de las flores periféricas $\leq 16$ cerdas; vilano de las flores del disco $\geq 22$ cerdas P. baccharis (Mill.) Pruski

Pluchea baccharis (Mill.) Pruski, Sida 21(4): 2035. 2005.

E Conyza baccharis Mill., Gard. Dict. (ed. 8) no. 16. 1768. TIPO: MÉXICO. Campeche, Grows naturally at Campeche, s.f., Anónimo s.n. (lectotipo: BM-000833507!, designado por Pruski, 2005).

= Pluchea eggersii Urb., Symb. Antill. 3(3): 407. 1903. TIPO: REPÚPLICA DOMINICANA. Santo Domingo, Hab. in Sto. Domingo, in Savana de Cupey prope Loma Isabel de la Torre, 30.VI.1887, H. F. A. Eggers 2681 (holotipo: M-0029810!, isotipo: K-000222148!).
= Pluchea rosea R.K. Goodfrey, J. Elisha Mitchell Sci. Soc. 68(2): 266. 1952. TIPO: ESTADOS UNIDOS DE AMÉRICA. Florida, Lake Co., lake shores, vicinity of Eustis, 16-31.V.1894, G. V. Nash 758 (holotipo: GH-00011327!, isotipos: F-0051021!, MICH-1107630!, NDG-57692!, NY-00232534!, US-00129411!).

Hierbas perennes, $0.25-1 \mathrm{~m}$ de alto; tallos sin alas; ramas tomentulosas, rara vez pilosas y glandulares; hojas sésiles, láminas oblongas u oblongo-lanceoladas, 3.1-5.7 × 1.1-2.4 cm, ápice agudo o apiculado, base abrazadora, margen dentado, pilosas y glandulares en ambas superficies; inflorescencias corimbiformes con (5-)8-10 cabezuelas, pedúnculos individuales 1-6 mm de largo, tomentosos; involucro campanulado 5-7.7 × 6.2-7.8 mm; filarios 28-34, 3-4 seriados, imbricados, elípticos o lanceolados, ápice agudo, piloso-glandulares; flores periféricas $120-130$, corolas 3.4$3.7 \mathrm{~mm}$ de largo, cipselas 0.62-0.7 mm de largo, pardas, pilosas; vilano con 14-16 cerdas capilares, 2.6-3 mm de largo; flores del disco 24-32, corolas 4.8-5.6 mm de largo, rosadas, lóbulos ovado-lanceolados, glandulares en el ápice; anteras 2.1-3 mm de largo; estilo 5.3-6.2 mm de largo; cipselas 1-1.2 mm de largo, pardas o negras, pilosas; vilano con 2225 cerdas capilares, (4.1-)5-5.6 mm de largo. (Fig. 1B).

Comentarios taxonómicos: al publicarse Pluchea baccharis, nueva combinación para el basónimo Conyza baccharis (Pruski, 2005); el nombre P. rosea cae en sinonimia, aunque Goodfrey (1952) lo acuñó y publicó válidamente. Pluchea rosea agrupó especímenes incluidos en el complejo P. foetida (Goodfrey, 1952), los cuales presentaban caracteres vegetativos similares; entre ellos, hábito herbáceo perenne, hojas sésiles, oblongas, con la base truncada o auriculada. No obstante, diferían de $P$. foetida en algunas características de estructuras reproductivas, como la forma del ápice de los filarios (obtuso vs. acuminado), color de las flores (blanquecinas vs. rosadas) y longitud de la corola ( $\geq 8$ $\mathrm{mm}$ vs. $\leq 5 \mathrm{~mm}$ ).

Distribución: en México, Pluchea baccharis se encuentra en Campeche, Oaxaca, Quintana Roo, Veracruz y Yucatán, en elevaciones de 0-1110 m s.n.m. (Fig. 3). 
Hábitat: bosques de pino-encino, sabanas, vegetación de dunas costeras, hidrófila y secundaria; en suelos arenosos o arcillosos.

Fenología: florece y fructifica de junio a octubre y en enero.

Ejemplares examinados: MÉXICO. Campeche, municipio Candelaria, Pedro Barandas, s.f., C. M. Castro 65 (MEXU); loc. cit., s.f., C. M. Castro 66 (MEXU). Municipio Carmen, camino a Justo Sierra, después del Río Candelaria, C. Chan 3754 (MEXU); salida de Nueva Coahuila rumbo a Justo Sierra, 26.VI.1984, A. Puch 1327 (MEXU). Oaxaca, municipio San Miguel Chimalapa, La Hondonada, $16 \mathrm{~km}$ al S de Benito Juárez, valle del Río Portamonedas, $25 \mathrm{~km}$ en línea recta al NNE de San Pedro Tapanatepec, 4.I.1986, S. Maya 2686 (MEXU). Quintana Roo, municipio Felipe Carrillo Puerto, km 48 del camino Carrillo Puerto - Vigía Chico, 22.VI.1983, $R$. Durán e I. Olmsted 254 (MEXU). Municipio José María Morelos, Lake Chichancanab, s.f., W. C. Steere 2407 (F, US). Municipio Lázaro Cárdenas, Tintal, 19.VIII.1981, C. Chan y V. Rico 786 (MEXU); El Tintal, carretera Valladolid - Puerto Juárez, 19.VIII.1981, V. Rico y C. Chan 392 (MEXU). Veracruz, municipio Las Choapas, $11 \mathrm{~km}$ del entronque Las Choapas con la carretera Cárdenas - Coatzacoalcos, 16.VI.1973, A. D. Orozco 161 (MEXU). Municipio Tecolutla, Paso del Río, Río Riachuelos, 16.X.1982, Brigada de dunas (Facultad de Ciencias) 915 (MEXU). Yucatán, municipio Hunucmá, 21 km al NW de Hunucmá hacia Sisal, 19.X.1985, E. Estrada 333 (MEXU).

Pluchea carolinensis (Jacq.) G. Don, Hort. Brit. (ed. 3) 350. 1839.

三 Conyza carolinensis Jacq., Icon Pl. Rar. 3(1): pl. 585. 1788. TIPO: ESTADOS UNIDOS DE AMÉRICA. Carolina, Crescit sponte in Carolina, s.f., Anónimo s.n. (lectotipo: ilustración de C. carolinensis, Icones vol. 3 pl. 585, designado por Gillis, 1977).

= Conyza cortesii Kunth, Nov. Gen. Sp. (folio ed.) 4: 59. 1820. TIPO: MÉXICO. Morelos, Crescit propem villa Ferd. Cortesii et urbem Cuernavaca Mexicanorum, IV.1803, F. W. H. A. Humboldt y A. J. A. Bonpland 3982 (holotipo: P-00322299!, isotipo: P-00659906!).

EPluchea cortesii (Kunth) DC., Prodr. 5: 452. 1836.

= Pluchea odorata (L.) Cass. var. brevifolia Kuntze, Revis. Gen. PI. 1: 357. 1891. TIPO: VENEZUELA. Distrito Federal, Caracas, V.1874, C. E. O. Kuntze 1430 (holotipo: NY00232542!).

Arbustos, rara vez arborescentes, 0.5-4(-6) m de alto; tallos sin alas; ramas tomentulosas o pilosas, a veces glabrescentes con la edad; hojas con peciolos $0.7-2.3 \mathrm{~cm}$ de largo, láminas elípticas u ovado-elípticas, 5.8-18.6 × 1.9-7.7 $\mathrm{cm}$, ápice agudo, base cuneada, margen serrado, pilosas en ambas superficies; inflorescencias corimbiformes con 56-568 cabezuelas, pedúnculos individuales $0.4-1.5 \mathrm{~cm}$ de largo, pilosos; involucro campanulado 4.2-7 × 7.3-11.2 mm; filarios 50-54(-58), 5-6 seriados, imbricados, elípticos, ápice agudo, pilosos y glandulares; flores periféricas 148-186, corolas 3.4-4 mm de largo, cipselas 0.6-0.8 $\mathrm{mm}$ de largo, pardas o negras, pilosas; vilano con 12-15 cerdas capilares, 3.34(-4.8) $\mathrm{mm}$ de largo; flores del disco 28-40, corolas 3.8-5.3 $\mathrm{mm}$ de largo, rosadas, lóbulos oblongo-ovados, glandulares en el ápice; anteras (1.5-)2-3 mm de largo; estilo (5-)5.8-8 $\mathrm{mm}$ de largo; cipselas 0.6-0.9 mm de largo, pardas o negras, pilosas; vilano con 8-12 cerdas capilares, (2.7-)3-4.5 mm de largo (Fig. 4).

Comentarios taxonómicos: la historia nomenclatural de Pluchea carolinensis ha sido confusa a través del tiempo. A finales de la década de 1970, Gillis (1977) propuso el nombre $P$. symphytifolia (Mill.) Gillis y designó como lectotipo un ejemplar de BM (1009501!), pero años después, se observó que dicho espécimen corresponde a Neurolaena lobata (L.) Cass., especie de la tribu Neurolaeneae (Khan y Jarvis, 1989), la cual no está relacionada con Pluchea (Panero y Crozier, 2016).

Distribución: en México, Pluchea carolinensis se encuentra en Baja California Sur, Campeche, Chiapas, Chihuahua, Ciudad de México, Coahuila, Colima, Estado de México, Guanajuato, Guerrero, Hidalgo, Jalisco, Michoacán, Morelos, Nayarit, Nuevo León, Oaxaca, Puebla, Querétaro, Quintana Roo, San Luis Potosí, Sinaloa, Sonora, Tabasco, Ta- 


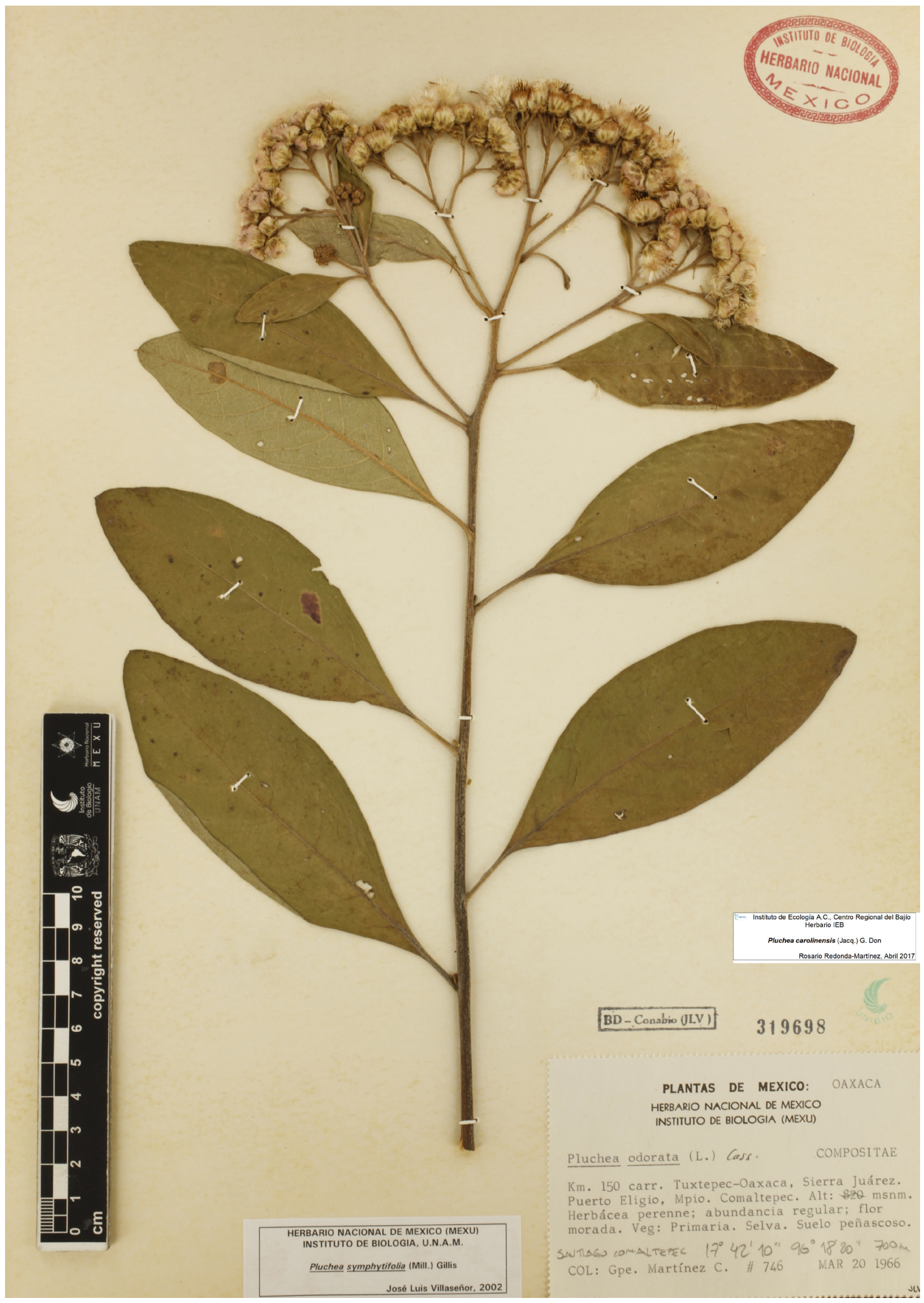

Figura 4: Ejemplar herborizado de Pluchea carolinensis (Jacq.) G. Don. 
maulipas, Veracruz y Yucatán, en elevaciones de 0-2450 m s.n.m. (Fig. 5).

Hábitat: bosques mesófilos de montaña, escuamifolios, de pino-encino, encinares, manglares, matorrales xerófilos, palmares, pinares, selvas altas perennifolias, bajas caducifolias, vegetación de dunas costeras, halófila, hidrófila, secundaria y ecotonos. En suelos arenosos, arcillosos, calizos o pedregosos, en ocasiones gipsófilos o derivados de areniscas y rocas sedimentarias.

Fenología: florece y fructifica durante todo el año.

Ejemplares examinados: MÉXICO. Baja California Sur, municipio Loreto, Cañón de Matancita near foot of Cuesta de Alta Gracia, 3.XI.1964, A. Carter 4897 (MEXU); Primera Agua, Sierra Giganta, 19.IV.1938, H. S. Gentry 3717 (MEXU, MO). Municipio Los Cabos, San José del Cabo, III.1897, A. W. Anthony 338 (F, MO); estero San José, 28.IV.1993, M. Domínguez 512 (IEB); Río San José, San José del Cabo, 24.II.1990, G. Zavala 45 (IEB). Municipio Mulegé, San José de Gracia, 2.IV.1989, R. Encarnación 209 (MEXU); Mulegé, 8.IV.2000, E. Guízar 4873 (MEXU). Campeche, municipio Calakmul, $1 \mathrm{~km}$ al O de G.E. Castellot no. 2 carretera Xpujil - Escárcega, 21.IX.2002, D. Álvarez 2076 (MEXU); Calakmul, s.f., G. F. Gaumer 1743 (F); $6 \mathrm{~km}$ al S de la entrada a Calakmul, 17.X.1997, E. M. Lira et al. 228 (MEXU, MO); $6 \mathrm{~km}$ al S de la entrada a Calakmul, 17.X.1997, E. Madrid et al. 196 (MEXU, MO); $6 \mathrm{~km}$ al S de la entrada a Calakmul, 16.X.1997, E. Martínez S. et al. 28896 (MEXU); km 9.5 de la terracería a las Ruinas de Calakmul, que está a $2 \mathrm{~km}$ al E de Conhuas, 3.Il.1983, O. Téllez et al. 6265 (MEXU). Municipio Calkini, Remate, 4 km rumbo a Isla Arenas, 25.II.1986, E. Ucán 4457 (MEXU); San Joaquín, 5 km de Celestún, 9.IV.1986, M. Ventura 483 (MEXU). Municipio Campeche, Bonfil, 3.III.1995, B. Faust y P. Ucan 379 (MO); alrededores de Chuina, $40 \mathrm{~km}$ de Champotón en la carretera a Escárcega, 9.V.1983, E. Góngora 400 (MEXU); zona E del predio del Centro de Recria F-1, 18.XII.1988, P. Juárez 779 (MEXU); predio agrícola por camino rústico km 2.5 Centro de Recria F-1-Thernucuy, 21.XII.1998, C. Martín 791 (MEXU); San Antonio Ebula, 23. II.2003, C. Pavón 467 (MEXU); Tikinmul on Mérida - Campeche road, 28.II.1958, B. G. Schubert y A. Gómez 1682
(MEXU). Municipio Carmen, carretera Sabancuy - Días Ordaz, 8.II.1982, C. Chan 1171 (MEXU); Pich, 22.I.1995, B. Faust y P. Ucan 357 (MO). Municipio Champotón, Conhuas, $165 \mathrm{~km}$ E of Escárcega on Méx. 186 along roadside W of village, 7.III.1983, R. A. Bye et al. 12008 (MEXU); Chuina, 9.V.1983, C. Chan 2262 (MEXU); Canasayab, 20.III.1932, C. L. Lundell 1418 (F); $8 \mathrm{~km}$ al S de Dos Naciones, camino a J. M. Morelos, El Civalito, 10.III.2002, E. Martínez S. et al. 35302 (IEB, MEXU); $5 \mathrm{~km}$ al S de ejido Ley de Fomento Agropecuario, camino a Dos Naciones, 10.III.2002, E. Martínez S. et al. 35425 (MEXU); camino Yohaltum - Paraíso, ca. 1 km antes de Paraíso, 31.I.1982, A. Puch et al. 230 (MEXU). Municipio Escárcega, km 10 al S del ejido Matamoros, 29.I.1985, C. Chan 4501 (MEXU); margen derecho del camino de terracería al ejido Venustiano Carranza, a $500 \mathrm{~m}$ de la carretera federal Escárcega - Champotón, 2.II.1995, C. Martín 311 (MEXU). Municipio Hopelchén, Zoh-Laguna, $10 \mathrm{~km}$ al $\mathrm{N}$ de Xpujil, sobre el camino a Dzibalchén, 1.Il.1996, P. Alvarado y G. Bacab 172 (MEXU); $2.44 \mathrm{~km}$ al N de Pachuitz, 30. III.2004, D. Álvarez 8544 (MEXU); Zoh-Laguna, por la laguna del pueblo, 30.VIII.1997, G. Bacam 19 (MEXU); $2.9 \mathrm{~km}$ al NE Río Pioneros, 17.Il.2002, J. Calónico et al. 21536 (MEXU); $0.66 \mathrm{~km}$ al $\mathrm{O}$ de Flores Magón, 12.III.2002, J. Calónico et al. 22707 (MEXU); $6 \mathrm{~km}$ al SE de Plan de Ayala, 17.II.2002, J. Calónico et al. 21607 (MEXU); $21 \mathrm{~km}$ al N de Narciso Mendoza poblado La Lucha, 21.II.2002, J. Calónico et al. 21903 (IEB, MEXU); $32 \mathrm{~km}$ al S de La Malacolona, 9.III.2002, J. Calónico et al. 22642 (MEXU); Chunchintok, 5.IV.1983, C. Chan 2007 (MEXU); 1 km de la carretera Chunchintok - Xmaben, 6.IV.1983, E. Góngora 185 (MEXU); Reserva de la Biosfera de Calakmul, 35 km de la entrada a la Reserva, 17.X.1997, E. M. Lira et al. 262 (MEXU, MO); sobre el km 24 de la carretera Xpujil - Campeche, 24.IV.1998, E. M. Lira et al. 684 (MEXU); $34 \mathrm{~km}$ al $\mathrm{S}$ de la caseta de entrada a Calakmul, 18.X.1997, E. Madrid y E. M. Lira 273 (MEXU, MO); km 3 al $S$ de Xcan-ha, camino a Xpujil, 23.II.1998, E. Madrid et al. 680 (MEXU); $13 \mathrm{~km}$ al S de Xca-ha, 30.IV.1998, E. Madrid et al. 759 (MEXU, MO); Xca-ha, $73 \mathrm{~km}$ al $\mathrm{N}$ de Xpujil, camino a Dzibalchen, 11.X.1997, E. Martínez S. et al. 28672 (MEXU); $34 \mathrm{~km}$ al S de la caseta de entrada a Calakmul, 16.X.1997, E. Martínez S. et al. 28955 (MEXU); La Aguada, $2 \mathrm{~km}$ al O de Calakmul, 17.X.1997, E. Martínez S. et al. 29027 (MEXU); 15 $\mathrm{km}$ al E de Xpujil, camino a Chetumal, en el rancho Las Deli- 


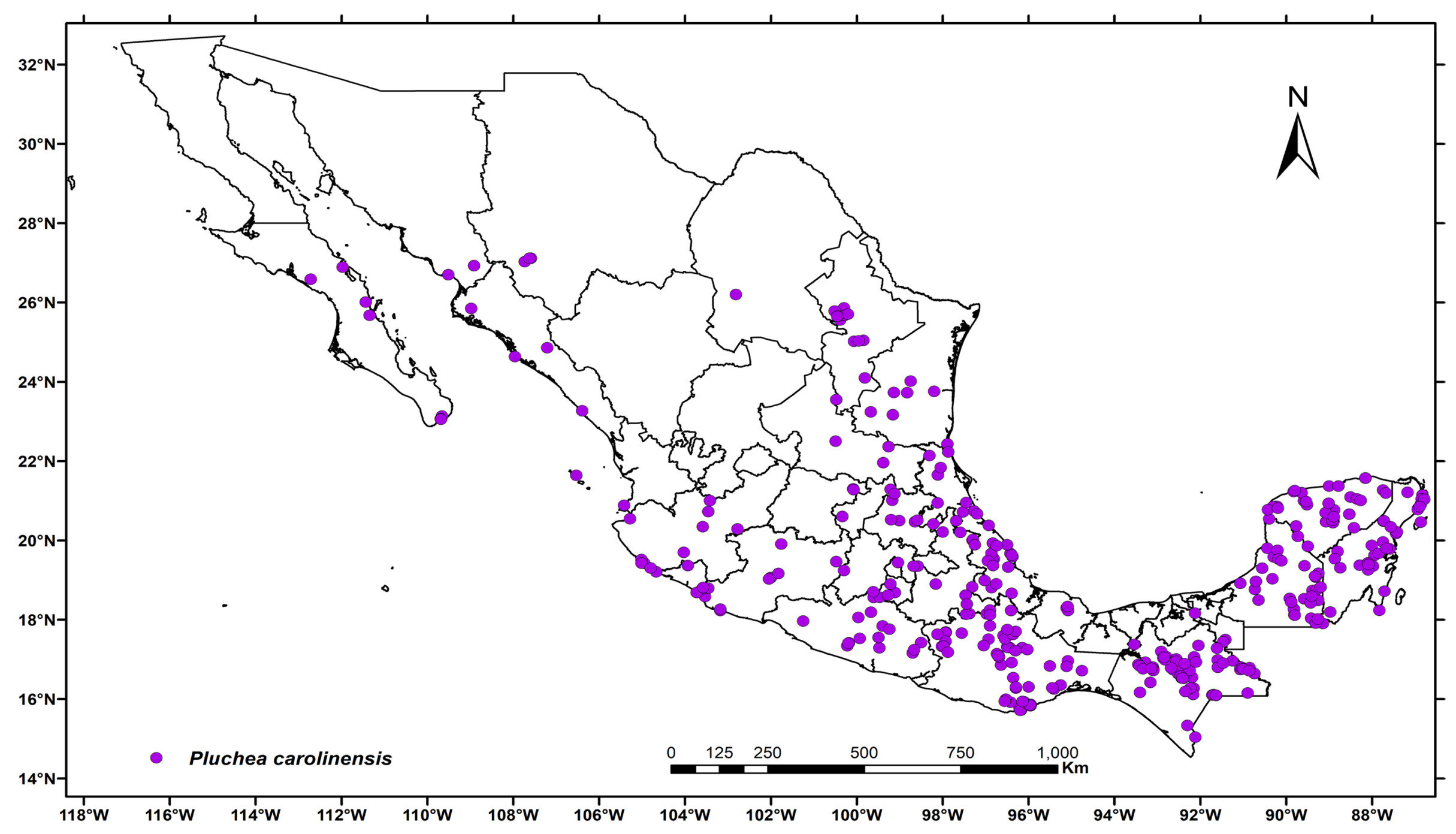

Figura 5: Mapa de distribución geográfica en México de Pluchea carolinensis (Jacq.) G. Don. 
cias, 21.II.2002, E. Martínez S. et al. 35050 (MEXU); 7 km al $S$ de la frontera Yucatán - Campeche, cerca de San Antonio Yaraxché, 10.II.2005, J. L. Tapia 1554 (MEXU). Municipio Palizada, km 30 de la desviación carretera Escárcega - Villahermosa, rumbo a Palizada, 25.V.1985, C. Chan 4689 (MEXU). Chiapas, municipio Amatenango del Valle, Barrio Amarillo, $3 \mathrm{~km}$ al E de Amatenango del Valle, carretera a Teopisca, 12.II.1988, M. Gómez 305 (MEXU); Yax Ja', 6 km al E de Amatenango del Valle, 17.IV.1988, M. Gómez 423 (MEXU); La Campana, $3 \mathrm{~km}$ al O Amatenango, carretera a Teopisca, 10.II.1988, J. López 272 (MEXU); near the center of Amatenango del Valle, 26.IX.1966, A. Shilom 1246 (F, NY). Municipio Berriozábal, $13 \mathrm{~km} \mathrm{~N}$ of Berriozábal near Pozo Turipache and finca El Suspiro, 9.X.1971, D. E. Breedlove 20212 (MEXU, MO). Municipio Bochil, along highway 195 from Ixtapa to Pichucalco, ca. $5 \mathrm{mi} \mathrm{N}$ of Bochil, 16.II.1979, T. B. Croat 47693 (MO). Municipio Chalchihuitán, Balum Aco', 24.IV.1988, E. Hernández 50 (MEXU); Cerro de Suy, K'an'ech', 24.VIII.1987, M. Pérez 50 (MEXU, MO); Majyeb Pom, $2 \mathrm{~km}$ al S de la escuela, 17.II.1988, M. Pérez 251 (MEXU, MO). Municipio Chamula, near school house of Yal Ichin, 26.VIII.1964, D. E. Breedlove 7142 (F). Municipio Chiapa de Corzo, along highway 195 between Chiapa de Corzo and Pichucalco, $7.9 \mathrm{mi}$ N of Bochil, 17.II.1987, T. B. Croat y D. P. Hannon 62092 (MO). Municipio Chilón, carretera de Palenque - Ocosingo km 73, 20.II.1983, A. Méndez 5525 (MEXU, MO). Municipio Comitán de Domínguez, 1 km al NE de Tierra Blanca, 20.II.1995, S. Ochoa et al. 4582 (MEXU); 3 mi NW of Comitán, 10.VIII.1962, G. L. Webster et al. 12912 (MEXU). Municipio Huixtán, 50 km antes de Ocosingo, viniendo desde San Cristóbal, 28.IX.1989, M. González et al. 896 (MEXU). Municipio Jitotol, along highway 195 between Ixtapa and Pichucalco, $5 \mathrm{mi}$ SE of Jitotol, 17. II.1979, T. B. Croat 47724 (F, MEXU, MO); Francisco I. Madero, 27.V.1999, C. Osorio 22 (MEXU). Municipio La Independencia, along gravel road at $\mathrm{km} 1.7$ leading to Dos Lagunas (from main blacktop road to Lagos de Montebello), 27.I.1979, T. B. Croat 46548 (MO). Municipio La Trinitaria, Los Lagos, $3 \mathrm{mi}$ NW of rancho San José, which is $34 \mathrm{mi}$ SE of Comitán, 18.I.1952, M. C. Carlson 2244 (F, MEXU); 7 km al E de Tziscao, 9.VIII.1985, T. Chehaibar 188 (MEXU); along road past Lagos de Montebello, where the road dead-ends, $5 \mathrm{mi}$ past the end of the blacktop, 17.I.1979, T. B. Croat
46511 (MEXU, MO); Lagunas de Montebello, 8.X.1978, W. Schwabe y W. Kailing s.n. (MEXU); 36-38 km al E de Tziscao, 7-9 km al E de Amparo Agua Tinta, camino Lagunas de Montebello - Bonampak, 8.IV.1983, J. L. Villaseñor y O. TéIlez 423 (MEXU). Municipio Las Margaritas, E of Comitán de Domínguez, along road just $\mathrm{N}$ of Lago Tziscao, $0.7 \mathrm{mi} \mathrm{E}$ of the park entrance, 7.I.1984, S. Sundberg 2420 (MEXU). Municipio Motozintla, Álvaro Obregón, $6 \mathrm{~km}$ al $\mathrm{S}$ de El Rosario, 5.II.1988, E. Ventura y E. López 5048 (ENCB, IEB, MEXU, $\mathrm{MO})$. Municipio Ocosingo, Mensura de Nuevo Guerrero con Nuevo Tumbalá, 10.III.2003, G. Aguilar 126 (MEXU); 28.8 km al SE de Nuevo Guerrero, 1.III.2003, J. Calónico et al. 22193 (MEXU); loc. cit., 1.III.2003, J. Calónico et al. 22197 (MEXU); Comunidad Lacandona de Nahá a $27 \mathrm{~km}$ al SE de Palenque, por la carretera fronteriza hasta el crucero Chancalá, después $55.6 \mathrm{~km}$ por el camino de terracería hacia Monte Líbano, 20.III.1997, A. Durán y S. Levy 456 (MEXU); $3.6 \mathrm{mi} \mathrm{N}$ of Ocosingo on road to Palenque, 6.III.1985, M. Luckow 2561 (MEXU); ejido Nueva Palestina, 15 km al NO, 29.II.1996, A. Luna et al. 121 (MEXU); $4 \mathrm{~km}$ al O de Crucero Corozal, camino Palenque - Boca Lacantum, 10.VIII.1984, E. Martínez S. 6860 (IEB, MEXU, MO); $5 \mathrm{~km}$ al S de Frontera Echeverría, sobre la orilla del Río Usumacinta, 4.XII.1984, E. Martínez S. 9046 (MEXU); $2 \mathrm{~km}$ al O de Crucero Corozal, camino Palenque - Boca Lacantum, 13.II.1985, E. Martínez S. 10117 (MEXU); $15 \mathrm{~km}$ al NO de Boca Lacantum camino a Palenque, 10.IV.1985, E. Martínez S. 11888 (MEXU); 14 km al NO de Crucero Corozal, sobre el camino Palenque - Boca Lacantum, 23.I.1986, E. Martínez S. 16628 (MEXU, MO); Crucero Corozal, sobre el camino Palenque - Boca Lacantum, 22.IV.1986, E. Martínez S. 17736 (MEXU); Centro Arqueológico Bonampak, 10.III.1982, J. Meave et al. 304 (MEXU); orilla del Río Lacantúm, 4 km arriba de la unión con el Río Cheyul, 15.V.1976, G. Siapiro y D. Elliot 469 (MEXU). Municipio Ocozocuautla de Espinosa, carretera Ocozocoautla - Malpaso, frente a la salida del sendero Bosque, 15.III.1998, C. Escobar 1083 (MEXU); carretera Ocozocoautla - Apic-Pac, km 12, 2.II.2000, A. Miranda y A. Zenón 1702 (MEXU); 1 km al NO del entronque Aeropuerto - Ocozocoautla - México, sobre la carretera 190, 3.III.1988, A. Reyes et al. 437 (MEXU). Municipio Oxchuc, Chenpil, $6 \mathrm{~km}$ al $O$ de Oxchuc, carretera internacional, 8.VI.1988, F. Gómez 213 (IEB, MEXU, MO); Cascada Nesbiljas, 10.X.1985, A. Méndez 
8506 (MEXU); Paraje Corralito, entre la carretera Oxchuc Ocosingo, 10.I.1986, A. Méndez 8716 (MEXU); route 186 from Palenque $19 \mathrm{mi} \mathrm{W}$ of Ocosingo, $32.8 \mathrm{mi} E$ of junction with route 190, 25.XII.1991, J. A. Soule y L. A. Prather 2965 (MEXU, MO). Municipio Palenque, disturbed area off road from Palenque to Agua Azul, near km post 11, 1.III.1975, W. S. Hoover 126 (MO); ejido Ángel Albino Corzo II, 15.VII.1993, B. Lukasser 46 (MEXU). Municipio Pichucalco, Selva Negra 6 km al NE de Rincón Chamula, carretera a Pichucalco, 26. III.1984, P. Tenorio et al. 5644 (MEXU). Municipio Pueblo Nuevo Solistahuacán, $2.5 \mathrm{mi}$ NE of Pueblo Nuevo Solistahuacán, 8.IX.1963, R. A. Marin 83 (F). Municipio San Cristóbal de las Casas, $1.6 \mathrm{mi} \mathrm{S}$ of Belem on route 190 (S of San Cristóbal), 20.XI.1977, J. La Duke et al. 552 (ASU, F). Municipio San Juan Cancuc, San Juan Cancuc, 16.I.1992, J. Brett 663 (MO); Krusil Jas cerde de la escuela $3 \mathrm{~km}$ del pueblo de Cancuc, 21.XII.1987, E. Sántiz 333 (MEXU). Municipio Tenejapa, Arroyo Jeshab, 5.III.1983, A. Méndez 5588 (IEB, MEXU, MO). Municipio Teopisca, SE city limits of Teopisca, 23. VII.1965, D. E. Breedlove 11289 (F); $8.2 \mathrm{mi}$ S of the junction of routes 199 and 190, on route 190 towards Guatemala, $4.9 \mathrm{mi} \mathrm{N}$ of the zocalo at Teopisca km post 109, 7.I.1990, J.

A. Soule et al. 2099 (MEXU). Municipio Tuxtla Gutiérrez, nuevo Zoológico Luis Álvarez del Toro, al NE de la ciudad de Tuxtla Gutiérrez por la carretera a San Cristóbal de las Casas, 26.IV.1983, J. I. Calzada 9599 (MEXU); 11.4 km N of Tuxtla Gutiérrez, Parque Nacional Cañón del Sumidero, 8. IV.1993, J. L. Neff 14 (MEXU); El Zapotal, al SE de Tuxtla Gutiérrez, 6.III.1987, E. Palacios 218 (MEXU); loc. cit., 6. III.1987, E. Palacios 248 (MEXU); canyon along freeway in North - Central Tuxtla Gutiérrez, ca. $5 \mathrm{~km}$ W of the University, 20.II.2007, J. Pruski et al. 4180 (MO). Municipio Tzimol, 15 km al O de Tzimol, camino a Pujiltic, 14.II.1987, E. Martínez S. 20059 (MEXU); $5 \mathrm{~km}$ al SE del entronque Tzimol Uninajab, camino a Uninajab, 24.II.1988, A. Reyes et al. 368 (MEXU). Municipio Unión Juárez, Chiquihuite, 15 km SE, 6.IV.1987, E. Ventura y E. López 4435 (ENCB, IEB, MEXU). Municipio Villaflores, Rancho Tierra Blanca, $50 \mathrm{~km}$ al SE de Tuxtla Gutiérrez, Carretera a Villa Flores, 28.III.1984, P. Tenorio et al. 5685 (MEXU). Chihuahua, municipio Batopilas, $S$ side of Barranca de Batopilas, W of La Bufa near Arroyo Bakossiachi, 23.IV.1973, R. A. Bye 3604 (MEXU); vicinity of La Bufa on S side of Barranca Batopilas, 26.III.1979, R. A.
Bye et al. 9261 (MEXU); Batopilas, XI.1980, Fierros 918 (MEXU). Ciudad de México, alcaldía Venustiano Carranza, terreno baldío al E del Aeropuerto Internacional y cerca de la Vía Tapo, cerca del límite de la delegación Venustiano Carranza, 30.XI.2000, H. Vibrans 2000 (MEXU). Coahuila, municipio San Pedro, Sierra Las Delicias, Cañón Agua Grande, ejido Nuevo Delicias, 23.X.1993, M. A. Carranza 1969 (MEXU). Colima, municipio Manzanillo, near km 292, $15 \mathrm{mi}$ ESE of Manzanillo, 23.VII.1957, R. McVaugh 15686 (MEXU). Municipio Minatitlán, 4-5 km SO de Platamarillos, 11-12 km ESE de Minatitlán, 1-2 km al O de Estanques, 22.V.1991, L. Guzmán y R. Cuevas 1180 (MEXU). Municipio Tecomán, Boca de Apiza, por Cerro de Órtiga, 16.X.1990, M. Cházaro et al. 6443 (IEB). Estado de México, municipio Santo Tomás Santa Bárbara, 1.II.1953, E. Matuda 28068 (MEXU). Guanajuato, municipio Xichú, 1 km del poblado del Álamo, sobre la carretera vecinal San Luis de La Paz - Victoria - Xichú, 3. III.2011, J. I. Calzada 25488 (MEXU); Misión de Santa Rosa, 24.II.1994, E. Pérez y E. Carranza 2893 (MEXU). Guerrero, municipio Apaxtla, $11 \mathrm{~km}$ al S de Apaxtla y cerca del km 46 de la carretera Teloloapan Apaxtla - El Caracol, 1.VII.2011, J. C. Soto 19039 (MEXU). Municipio Atoyac de Álvarez, 1 km al NE de San Antonio y aproximadamente $19 \mathrm{~km}$ al SW de EI Bálsamo, carretera Ciudad Altamirano - Zihuatanejo, 1. II.2012, J. C. Soto 20093 (MEXU); cerca de El Paraíso, 19. III.1985, J. C. Soto y S. Aureoles 7813 (IEB, MEXU); El Ranchito, $13 \mathrm{~km}$ al NE de El Paraíso, 21.III.1985, J. C. Soto y S. Aureoles 7898 (MEXU); 14 km al NE de Paraíso, 28.III.1983, J. C. Soto y E. Martínez S. 5097 (MEXU, MO). Municipio Chilpancingo de los Bravo, Jardín Botánico Chilpancingo, 14.X.1977, C. Toledo y M. Blanco 51 (MEXU); Rincón de la Vía, 21.III.1960, H. Kruse 225 (MEXU). Municipio Cocula, Apipilulco, cercanías de Iguala, 11.I.1955, S. Fuentes s.n. (MEXU). Municipio General Heliodoro Castillo, Yerbabuena, km 170 Atoyac - Xochipala, 1.II.1983, Laboratorio de Biogeografía 532b (MEXU). Municipio José Azueta, 1 km al NE de San Antonio y $19 \mathrm{~km}$ al SO de El Bálsamo, carretera Ciudad Altamirano - Zihuatanejo, 1.II.2012, J. C. Soto 20093 (MEXU). Municipio Malinaltepec, camino de Paraje Montero a Tlapa, 25.III.1982, E. Velázquez 118 (MEXU); Malinaltepec, 15.I.1992, I. Wagenbreth 830 (MEXU, MO). Municipio Mártir de Cuilapan, La Esperanza, 30.I.1996, M. J. Sánchez 30 (FCME, IEB, MEXU). Municipio Taxco de Alarcón, Ca- 
sahuatlán, 14 km al S, 22.I.1998, J. Calónico 7019 (FCME, MEXU); periferia O de la Ciudad de Taxco, en el borde de la carretera a Tetipac, 28.XII.1987, F. Terán y H. Alarcón 394 (MEXU). Municipio Tepecoacuilco de Trujano, $100 \mathrm{~m}$ de la autopista del Sol, 20.II.2004, R. Alvarado y J. Rojas 502 (FCME, MEXU). Municipio Tetipac, Cuitlapa, $3 \mathrm{~km}$ al NE, 26.II.1998, R. Cruz 1979 (FCME, MEXU). Municipio Tlapa de Comonfort, $33 \mathrm{~km}$ de Tlapa, rumbo a Metlatonoc, cerca de Petlalcala, sobre el lecho del río, 4.VI.1981, E. Velázquez 39 (MEXU). Hidalgo, municipio Cardonal, Barranca de Tolantongo, $2 \mathrm{~km}$ al $\mathrm{O}$ de las grutas, 3.IV.2005, A. García y S. Franco 7735 (MEXU). Municipio Ixmiquilpan, $5 \mathrm{~km}$ al $\mathrm{N}$ de Ixmiquilpan, XI.1981, R. Hernández 6626 (MEXU, MO). Municipio Jacala, mountains along route $85,12 \mathrm{mi} \mathrm{SW}$ of Hidalgo - San Luis Potosí state border, 23.III.1961, R. M. King y $R$. Johnson $4228(\mathrm{~F})$; rocky mountain side at Jacala, 26.VI.1939, V. H. Chase 7168 (MO). Municipio Pacula, Boquilla de Jiliapan, 28.II.2008, M. Martínez 7987 (IEB, QMEX). Municipio San Agustín Metzquititlán, $2 \mathrm{~km}$ al E de la barranca de Venados, 11.I.1980, R. Hernández 3935 (MEXU, MO); $10 \mathrm{~km}$ al E de Metzquititlán, 24.III.1981, R. Hernández y D. Rodríguez 5591 (MEXU, MO). Municipio Tecozautla, Taxido, 3.V.1991, A. Ortíz 53 (IEB). Municipio Tenango de Doria, 9 $\mathrm{km}$ al E de Tenango de Doria, hacia Tutotepec, 21.II.1981, $R$. Hernández y D. Rodríguez 5545 (MEXU, MO). Jalisco, municipio Cihuatlán, E of Barra de Navidad, 7.IV.1951, $R$. McVaugh 11836 (MEXU). Municipio Jamay, ribera $\mathrm{N}$ del Lago de Chapala, 13.VI.1983, F. Estrada s.n. (MEXU). Municipio La Huerta, Arroyo Hornitos, Estación de Biología Chamela UNAM, 13.II.1998, J. Calónico 7761 (MEXU); La Manzanilla, 19.III.2007, G. Castillo et al. 10830 (MEXU); Salinas de Careyes, Costa Alegre, 13.III.1985, C. del Río 5 (MEXU); Salinas de Careyes a $7 \mathrm{~km}$ al SE de la Estación de Biología Chamela, carretera Puerto Vallarta - Barra de Navidad, 29.I.1986, E. J. Lott 2777 (MEXU, MO); rancho El Limbo, Cuixmala, 11.IX.1986, A. Pérez 1992 (MEXU); estero cerca de La Manzanilla, 25.III.1979, L. M. Villarreal y R. Guzmán 8528 (IEB). Municipio Puerto Vallarta, vicinity of Los Arcos, $3.5 \mathrm{mi} \mathrm{S}$ of Río Cuale, slopes near Shore, 20.X.1970, G. L. Webster y G. J. Breckon 15791 (MEXU). Municipio San Cristóbal de la Barranca, rancho El Escalón, 4.III.1987, R. Ornelas 821 (IEB, MEXU). Municipio Tuxcacuesco, slopes facing $\mathrm{E}$ toward Río Tuxcacuesco, arid foothills of Sierra de
Manantlán, slopes facing W toward Río Ayuquila. $6 \mathrm{~km}$ due $\mathrm{W}$ of Tuxcacuesco on road (just below saddle and $\mathrm{N}$ of) Camichín, $11.5 \mathrm{~km}$ (by air) due NE of Zenzontla, 15.III.1989, T. S. Cochrane et al. 11650 (IEB). Municipio Zapopan, Villa Universitaria, 12.III.1976, S. Larios s.n. (MEXU). Municipio Zoacalco, pasando Acatlán de Juárez, cerca de la desviación a San Marcos y Los Pocitos, 10.I.1987, M. Cházaro et al. 4380 (IEB, MEXU). Michoacán, municipio Aquila, highway 200 between La Placita and Playa Azul, $44.1 \mathrm{mi}$ SE bridge at La Placita, 7.III.1987, T. F. Daniel 4903 (MEXU); 5 km camino Aquila - La Placita, 28.VIII.1979, B. Guerrero 152 (MEXU); Laguna Colorada, $5 \mathrm{~km}$ al SW del Ranchito, 5.VII.1990, M. Illescas y O. Esparza 89 (MEXU). Municipio Coahuayana, Atenquique, $26 \mathrm{~km}$ al NE del Ranchito, 27.III.1981, J. C. Soto et al. 2814 (MEXU, MO); $3 \mathrm{~km}$ al E del Camalote, brecha Coahuayana - Villa Victoria - Coalcomán, 23.XII.1984, J. C. Soto et al. 7137 (MEXU); Boca de Apiza, 18.VII.1985, J. C. Soto et al. 9518 (MEXU); $13 \mathrm{~km}$ al SE de Palos Marías, 18.X.1988, J. C. Soto et al. 11148 (MEXU, NY). Municipio Jiménez, Alberca de los Espinos, 5.V.2014, R. Contreras 82 (IEB, MEXU). Municipio Lázaro Cárdenas, La Soledad, 28. III.1899, E. Langlassé 972 (F). Municipio Múgica, 5.5 km al NE de la salida a Nueva Italia por la autopista Lázaro Cárdenas - Morelia, al lado del Río El Marqués, 1.V.2006, V. W. Steinmann y Y. Ramírez 5391 (MEXU). Municipio Nueva Italia, alrededores de la presa General Francisco J. Múgica, Puente de Piedra, 18.IV.2009, M. Méndez et al. 11 (MEXU, MO); loc. cit., 28.VI.2009, M. Méndez et al. 135 (MEXU, MO). Municipio Tunhuato, $1 \mathrm{~km}$ al O de Tunhuato, por la carretera a La Barca, 21.XI.1989, S. D. Koch y P. A. Fryxell 218 (F). Municipio Zitácuaro, San José Purúa, 10.III.1984, J. C. Soto y A. Román 6221 (MEXU, MO). Morelos, municipio Amacuzac, along free route $95,8 \mathrm{mi} E$ of Huamintlan, 14.III.1961, R. M. King y R. Johnson 4167 (F, NY). Municipio Cuernavaca, Cuernavaca, Colonia Reforma, XII.1979, A. Ortega 14 (MEXU, MO). Municipio Jojutla, Lago de Tequesquitengo, vertiente W Playa de Roca, 28.II.1981, D. González 13 (MEXU). Municipio Tlaltizapán, Tlaltizapán, 12.III.1966, J. Vázquez 1753 (MEXU). Nayarit, municipio Bahía de Banderas, Playa Malpaso, $2 \mathrm{~km}$ al NE de Sayulita, 26.III.2002, $P$. Carrillo 3057 (IEB). Municipio Compostela, Playa Los Venados, 22.IV.1987, C. L. Díaz 18642 (IEB). Municipio San Blas, María Madre Island, 7.V.1897, E. W. Nelson 4181 (F). Nuevo 
León, municipio Aramberri, N of Aramberri, 23.XI.1989, J. C. Hinton 19965 (IEB, MEXU). Municipio Bustamante, road to Bustamante N of La Presita, $5.6 \mathrm{mi} \mathrm{NW}$ of hwy 70, 21.V.1982, L. J. Dorr y T. L. Atkins 2365 (ASU). Municipio Galeana, San José del Río, 23.III.1992, J. C. Hinton 21865 (IEB); La Poza - Río San José, 1.IX.1992, J. C. Hinton 22374 (IEB). Municipio Guadalupe, San Rafael, VII.1911, B. Abbon 104 (MO). Municipio Montemorelos, Las Pintas, $5 \mathrm{~km}$ aguas arriba de La Avanzada, junto al manantial La Virgencita, cañón del Río Pilón en la Sierra Madre Oriental, 14.X.1983, $R$. Sánchez 423 (MEXU). Municipio Monterrey, Monterrey, 14.VII.1924, G. L. Fisher s.n. (F, MO); Monterrey, river bottom 8.IX.1937, L. A. Kenoyer (F, MO); river banks Monterrey, VII.1883, C. G. Pringle 1896 (F); loc. cit., 2.VIII.1889, C. G. Pringle 2646 (F, MEXU, MO). Municipio Rayones, Río Pilón, 9.VI.1980, J. C. Hinton 17832 (IEB, MEXU); 7 km al O de Rayones, por el camino a Montemorelos, en el lecho del Río Pilón, 24.V.1982, R. Sánchez 102 (MEXU). Municipio Santa Catarina, Cañón Huasteca, Río Santa Catarina, 28.II.1946, T. Alonis et al. 151 (F); loc. cit., 16.VIII.1944, F. A. Barkley 14554A (F); Canyon Huasteca, 8-10 mi of Monterrey, 31.I.1952, H. S. Gentry 11523 (MEXU); Santa Catarina, Huasteca Canyon, 30.I.1940, I. K. Langman 2922 (MEXU); Cañón de Santa Catarina, X.1942, E. Lyonnet 3907 (MEXU); Huasteca Canyon, W of Monterrey and S of Santa Catarina, ca. $1 \mathrm{mi}$ from entrance of canyon, 2.VIII.1972, D. J. Pinkava et al. 9733 (ASU, DES). Municipio Villa de García, Río Pesquería, al E de Villa de García, 17.XI.2001, J. A. Encina y M. A. Llamas 1004 (MEXU). Oaxaca, municipio Candelaria Loxicha, $11 \mathrm{~km}$ al N de La Galera, carretera Pochutla - Miahuatlán, 20.I.1988, A. Campos 1112 (MEXU). Municipio Capulalpan de Méndez, SO del Río Natividad, 1.X.2003, S. Figueroa y F. Y. Guzmán $847 a$ (MEXU). Municipio Guelatao de Juárez, Guelatao bridge, 4.II.1966, W. R. Ernst 2447 (MEXU); Sierra de Juárez, Ruta 175, 2 km al SO de Guelatao de Juárez, 20.IV.1982, D. H. Lorence y R. Cedillo 4109 (MEXU, MO). Municipio Nuevo Soyaltepec, alrededores de la cortina de la presa Miguel Alemán, 21.III.1964, L. González 570 (MEXU). Municipio Oaxaca, San Felipe del Agua, 5.IV.2005, L. Cervantes 8 (MEXU); Oaxaca, XII.1900, C. Conzatti et al. 1122 (MEXU); Hacienda Guadalupe, 11.III.1937, C. Conzatti 5254 (MEXU). Municipio Pluma Hidalgo, 0.8 km en línea recta al SO de Pluma Hidalgo, 8.III.2011, R. Redonda et al.
374 (MEXU). Municipio San Agustín Loxicha, Buenavista Loxicha, 18.V.2003, A. Luna y B. Rendón 239 (IEB). Municipio San Andrés Yaá, $11 \mathrm{~km}$ al $\mathrm{S}$ de Villa Alta, hacia Tlacolula, 16.III.1982, R. Torres y R. Cedillo 132 (MEXU, MO). Municipio San Antonio Nanahuatipan, $2 \mathrm{~km}$ al $\mathrm{N}$ de Casa Blanca, 17.IV.1987, A. Salinas y C. H. Ramos F-3890 (IEB). Municipio San Juan Bautista Cuicatlán, km 168 de la carretera Cuicatlán - Oaxaca, 14.IV.2002, C. A. Cruz y E. Martínez S. 353 (MEXU); camino de San Juan Bautista Cuicatlán a Concepción Pápalo, 7.III.2014, R. Medina et al. 5873 (MEXU). Municipio San Juan Bautista Tuxtepec, Presa Miguel Alemán, Temascal, 10.III.1983, R. Torres et al. 2361 (MEXU). Municipio San Juan Guichicovi, San Juan Guichicovi, IV.1986, A. Nereyda y M. Heinrich 42 (MEXU). Municipio San Juan Mixtepec, mountains above San Juan Mixtepec, 15.XII.1996, E. Hunn 607 (MEXU); Cruz family garden, San Juan Mixtepec, 18.I.1997, E. Hunn 772 (MEXU); San Juan Mixtepec, 13. VII.1997, E. Hunn 1525 (MEXU). Municipio San Juan Quiotepec, Quiotepec, 15.I.1986, J. García 1 (MEXU). Municipio San Lorenzo Victoria, La Loma, $5 \mathrm{~km}$ al O de San Jorge Nuchita, hacia Yucuyachi, 7.II.1992, J. I. Calzada 18292 (MEXU). Municipio San Lucas Zoquiapan, 45 km de Teotitlán, hacia Huautla, 13.IX.1989, A. Flores 1510 (MEXU). Municipio San Martín Itunyoso, $11 \mathrm{~km}$ al SO de San Isidro Chicahuaxtla, carretera Tlaxiaco - Putla, 1.XII.1993, R. Torres 14208 (MEXU). Municipio San Miguel Chimalapa, Río Grande, 1 $\mathrm{km}$ riverdownward of San Miguel Chimalapa, 12.VI.2014, M. S. Greck 123 (MEXU). Municipio San Miguel del Puerto, O de Santa María Xadani, El Palmar, camino a La Blas, 19. II.2002, J. Pascual 359 (IEB, MEXU); Cerro del Machete, II.1941, B. P. Reko 6214 (F). Municipio San Pablo Etla, Las Salinas, $1.5 \mathrm{~km}$ al NE de la 3a sección de San Pablo Etla, 17.VIII.2006, E. Padilla 42 (MEXU). Municipio San Pablo Macuiltianguis, Paraje Ribera del Río Mariposas, Macuiltianguis, 4.V.1980, G. Pérez 191 (MEXU). Municipio San Pablo Villa de Mitla, vicinity of Mitla; valley of Río Grande de Mitla and nearby foot-hills, 1.VIII.1972, E. Messer 81b (MEXU). Municipio San Pedro Huamelula, $71 \mathrm{mi}$ W of Salina Cruz (junction of highway 200 and 185) on highway of Puerto Escondido, $1 \mathrm{mi}$ E of Río Chacalapa, 9.III.1985, M. Lavin et al. 5329 (MEXU). Municipio Santa Lucía del Camino, Santa Lucía del Camino, 30.VIII.1920, C. Conzatti 4043 (MEXU). Municipio Santa María Guienagati, 11 km al NO de Lachivi- 
za, brecha a Lachiguiri, 15.III.1986, P. Tenorio et al. 11135 (MEXU). Municipio Santa María Huatulco, Río Xúchitl, 1.93 km en línea recta, 201 grados $\mathrm{N}$ del Puente Xúchitl, 16.I.2005, A. Nava et al. 963 (MEXU); arroyo que desemboca en la Playa Arroyo. $5 \mathrm{~km}$ en línea recta y $358^{\circ}$ de la Playa Arroyo, 3.V.2005, A. Nava et al. 1039 (MEXU); Santa María Huatulco, 14.I.2005, A. Sánchez et al. 650 (MEXU); 1.92 km en línea recta, 201 grados del puente Xúchitl, 15.II.2005, $A$. Saynes et al. 5282 (MEXU, MO); Arroyo La Pozona, 0.33 km en línea recta de Playa Chachacual $\left(359^{\circ}\right)$, 2.V.2005, K. Velasco et al. 723 (MEXU). Municipio Santa María Petapa, Santa María Petapa, 21.V.1992, B. Frei 156 (MEXU). Municipio Santa María Teopoxco, road between Teotitlán del Camino and Huautla de Jiménez, 20.8 mi E of Teotitlán, 22. II.1979, T. B. Croat 48239 (MEXU, MO). Municipio Santa María Zoquitlán, orilla del Río Quiechapa, 6.III.1987, A. Flores 1182 (MEXU). Municipio Santiago Astata, Barra de la Cruz, 1 km E, por El Zapotal, 27.III.1998, M. Elorsa 60 (MEXU); Barra de La Cruz, Laguna Potrerón, 2.IV.1988, M. Elorsa 122 (MEXU); loc. cit., 23.XII.1998, M. Elorsa 1332 (MEXU); Zimatán, $0.5 \mathrm{~km} \mathrm{~N}$ del Puente, 8.I.1999, M. Elorsa 1441 (MEXU); Chancalapa, puente en la carretera costera, 0.5 km S, 14.I.1999, M. Elorsa 1477 (MEXU); Barra de la Cruz, 2 km NO, 4.I.2000, M. Elorsa 2605 (MEXU); Barra de La Cruz, 3 km N por el Río Chacalapa, 7.III.2000, M. Elorsa 2784 (MEXU); Barra de la Cruz, $500 \mathrm{~m}$ al SE, sobre el río, 9.I.2001, M. Elorsa 4152 (MEXU); Barra de La Cruz, 2 km al N, sobre el Río Chacala, 12.II.2001, M. Elorsa 4281 (MEXU); El Sanjón Grande, $200 \mathrm{~m}$ al E de la laguna de Barra de la Cruz sobre la playa, 23.II.2002, M. Elorsa 5784 (IEB, MEXU); Barra de la Cruz, 1 km SSE por el río, 1.II.1999, M. Elorsa y D. Scheidegger 1610 (MEXU); Chacalapa, puente en la carretera costera, $0.5 \mathrm{~km}$ al S, 6.II.1999, M. Elorsa y D. Scheiddeger 1695 (MEXU); Barra de la Cruz, pueblo, Tehuantepec, 1.II.1999, D. Scheidegger y M. Elorsa 14 (MEXU). Municipio Santiago Comaltepec, Santiago Comaltepec, 30.V.1987, L. Hernández 18 (MEXU); La Esperanza, 10.X.1988, R. López 375 (MEXU); Sierra de Juárez, km 150 carretera Tuxtepec - Oaxaca, Puerto Eligio, 20.III.1966, G. Martínez 746 (MEXU, MO). Municipio Santiago DominguiIlo, 16 km al SE de Dominguillo, por la carretera Tehuacán Oaxaca, 29.VIII.1980, F. Chiang et al. F-1827 (MEXU). Municipio Santiago Laxopa, Santiago Laxopa, 7.VI.1987, N.
Maldonado y G. J. Martin 143 (MEXU, MO); loc. cit., 21. VIII.1988, N. Maldonado y G. J. Martin 337 (MEXU). Municipio Santiago Juxtlahuaca, $5 \mathrm{~km}$ al $\mathrm{N}$ de Santiago Juxtlahuaca, desviación de la carretera Mesones - San Juan Mixtepec, 12.I.1995, J. I. Calzada 19616 (MEXU); 1.4 km de Santiago Juxtlahuaca, carretera a Santa Catarina Noltepec, 12. III.1998, J. I. Calzada 22413 (MEXU). Municipio Santo Domingo Tonalá, Barranca Yubijaza, 20.III.2009, L. A. Hernández y A. Torres 978 (MEXU); del Boquerón a Los Mangos, 8.I.2009, A. Torres y L .A. Hernández 841 (MEXU); Barranca Yubijaza hasta Los Limoncillos cuesta Camino Real, 1. II.2009, A. Torres y L. A. Hernández 903 (MEXU). Municipio Santo Domingo Tehuantepec, El Zacatal, Rancho Limón, 17 km al O de Tehuantepec, 18.II.1986, C. Martínez 419 (IEB, MEXU); $N$ de de Cerro Lieza, $4 \mathrm{~km}$ al NO de Tehuantepec, 16.V.1987, C. Martínez 976 (MEXU); Arroyo El Tepesco, 2 $\mathrm{km}$ al S del poblado, $37 \mathrm{~km}$ al O de Tehuantepec entrando por Pozo Zorrillo, 16.II.1988, C. Martínez 1332 (MEXU). Municipio Santo Domingo Tonaltepec, on route $190,11.7 \mathrm{mi} \mathrm{S}$ of Tonaltepec turnoff and $16.3 \mathrm{mi}$ of rte. 190, 15.VII.1990, J. A. Soule y D. R. Brunner 2255A (MO). Municipio Santo Tomás Jalieza, Río Pesquería, al E de Villa de García, 11. III.2006, C. A. Cruz et al. 2912 (MEXU). Municipio Santos Reyes Tepejillo, $8 \mathrm{~km}$ al $\mathrm{N}$ de Santos Reyes Tepejillo, entrada por La Cruz, Río Boquerón, 14.II.1995, J. I. Calzada 19699 (MEXU). Municipio Totontepec Villa de Morelos, Totontepec, 12.IV.1987, J. Rivera 724 (MEXU). Municipio Villa de Tamazulapan del Progreso, highway 190 between Oaxaca and Izúcar de Matamoros, $121 \mathrm{mi}$ NW of Oaxaca, $18.4 \mathrm{mi}$ ESE of Huajuapan de León, 23.II.1987, T. B. Croat y D. P. Hannon 65667 (MEXU, MO); Río del Oro, $5 \mathrm{~km}$ al NO de Tamazulapan, 12.IV.1987, A. J. García et al. 3103 (IEB); Ruta 190, cerca Río del Oro, 9.VIII.1985, D. H. Lorence y A. J. García 4823 (F, MEXU). Puebla, municipio Caltepec, Piedra de Afilar, al E de Coatepec por la barranca a Santa Lucía, 10. XII.2001, P. Tenorio y L. O. Alvarado 21657 (MEXU); Callejón del Cosahuico al S de Caltepec, 9.II.1984, P. Tenorio y C. Romero 5408 (MEXU). Municipio Chila, Chila, 25.III.1944, F. Miranda 3095 (MEXU). Municipio Huauchinango, Necaxa, 22.II.1962, L. Vela 500 (MEXU). Municipio Hueytamalco, campo experimental Las Margaritas, Instituto Nacional de Investigaciones Forestales Agrícolas y Pecuarias (INIFAP), 24.IV.2008, G. Cornejo et al. 2746 (IEB, MEXU); Rancho Las 
Margaritas, 7.VI.1976, W. López 179 (MEXU). Municipio San José Axusco, alrededores del Cerro Petlanco, 15.V.1986, A. Salinas y P. Solís F-3283 (IEB, MEXU). Municipio Nicolás Bravo, $22 \mathrm{~km}$ al S de Esperanza, por la carretera a Tehuacán, 19.I.1984, F. Chiang F-930 (MEXU). Municipio Puebla, Presa Valsequillo, 25.III.1967, L. W. Boege 534 (MEXU). Municipio Tehuacán, km 24 from the Cuacnopala highway heading towards Mexico, 15.V.2002, J. I. Calzada y F. Paredes 23202 (MEXU); Tehuacán, s.f., C. Patoni s.n. (MEXU); Municipio Tepexi, Tepexi, 19.IV.1970, L. W. Boege 1366 (MEXU). Municipio Tlacotepec de Benito Juárez, $24.5 \mathrm{~km}$ de la autopista México - Orizaba, sobre la autopista Tehuacán - Oaxaca, 8. IV.2004, O. Téllez et al. 17495 (MEXU). Municipio Venustiano Carranza, Río Cazones, III.1951, H. Bravo s.n. (MEXU); Agua Fría, 11.III.1986, A. D. González 14 (MEXU); María Andrea, 4.III.1986, A. D. González 19 (IEB); Río Cazones, III.1951, D. Ramírez 214 (MEXU). Municipio Zapotitlán, Texcala, aproximadamente $19 \mathrm{~km}$ al SW de Tehuacán, por la carretera a Huajuapan de León, 3.X.1982, J. L. Villaseñor y G. Delgado 273 (MEXU); afueras de San Antonio Texcala, rumbo a Tehuacán, 3.X.1980, J. L. Villaseñor et al. 70 (MEXU). Querétaro, municipio Arroyo Seco, 4 km de Arroyo Seco, sobre la carretera a Concá, 21.II.1989, E. Carranza 1490 (ENCB, MEXU). Municipio Cadereyta de Montes, Maconi, XI.1942, J. R. Bruff 1038 (MEXU). Municipio El Marqués, ladera $\mathrm{N}$ de La Cañada, cerca de El Manantial, 15. VIII.2002, A. Cabrera 178 (IEB). Municipio Jalpan de Serra, La Isla, al S de Tanchanaquito, 23.III.1993, L. López 536 (MEXU). Municipio Landa de Matamoros, curva de La Palmilla, 5 km al NW de La Vuelta, 23.II.1989, E. González 400 (MEXU); Puerto del Gato, $3 \mathrm{~km}$ al O de Acatitlán de Zaragoza, 13.III.1989, E. González 436 (MEXU); 6 km al SO de Santa Inés, 17.III.1985, R. Fernández 2769 (ASU, ENCB, MEXU); 10 $\mathrm{km}$ al $\mathrm{S}$ de Agua Zarca, brecha a Pisaflores, R. Fernández 3871 (ENCB); $11 \mathrm{~km}$ al SE de Agua Zarca, por el camino a Pisaflores, 3.III.1988, S. Zamudio 6251 (IEB, MEXU). Municipio Peñamiller, Junta de Higueras en la rivera del Río Estórax, cerca de Higueras, 9.II.1994, R. Hernández et al. 10471 (IEB, MEXU); $11 \mathrm{~km}$ al N de Peña Blanca, ladera E del cerro La Tembladera, 9.III.1978, S. Zamudio 2706 (ENCB, MEXU); $6 \mathrm{~km}$ al S de Peña Blanca, 18.II.1979, S. Zamudio 3651 (ENCB, MEXU). Municipio Pinal de Amoles, fondo del Cañón de Misión de Bucareli, 10.IV.2002, Y. Pantoja 199
(MEXU). Municipio Tolimán, between Tolimán and Pilón, 45-50 mi by road NE of Querétaro, 24.IV.1949, R. McVaugh y R. L. Wilbur 10372 (MEXU, MICH). Quintana Roo, municipio Benito Juárez, Jardín Botánico de Puerto Morelos, s.f., $S$. Escalante 10 (MEXU); zona hotelera de Cancún, Palmar VI, 14.VIII.1989, S. Escalante 608 (MEXU); Punta Nizua, entrada al Club Mediterráneo, 17.III.1981, I. Espejel y V. Rico 151 (MEXU); $6 \mathrm{~km}$ al Norte de Puerto Morelos, en la carretera a Cancún, 7.I.1980, M. Sousa et al. 10945 (MEXU). Municipio Cozumel, $1 \mathrm{~km}$ al $\mathrm{N}$ de la carretera transversal sobre el camino a la zona arqueológica de San Gervacio, Isla de Cozumel, 13.III.1986, E. Cabrera y O. Téllez 11050 (MEXU, MO); Macario Gómez, 14.IV.1987, E. Ukán y M. Poot 5127 (MO). Municipio Felipe Carrillo Puerto, Xkonhá, $4.5 \mathrm{~km}$ hacia Xhazil Sur, 16.X.1985, F. Balam 480 (MEXU); Mixtequilla, 4 km hacia Yoctzonot Poniente, 10.XI.1985, F. Balam 635 (MEXU); Reserva de la Biosfera, km 19 de la brecha Chumpon - Vigía Chico, 14.II.1988, E. Cabrera y H. de Cabrera 15494 (MO); 0-3 km al S de Las Palmas, aproximadamente $36 \mathrm{~km}$ al S de Felipe Carrillo Puerto, sobre la carretera a Chetumal, 17. II.1989, E. Cabrera y W. Colli 16331 (MEXU); 6-10 km al NE de Felipe Carrillo Puerto, sobre el camino a Vigía Chico, 3. III.1989, E. Cabrera et al. 16358 (MEXU); $13.5 \mathrm{~km}$ al E de Señor, 5.III.2002, J. Calónico y E. Martínez S. 22361 (MEXU); $1 \mathrm{~km}$ al $\mathrm{N}$ del Crucero de Chumpon, 15.II.1984, R. Durán e I. Olmsted 807 (MEXU); $2 \mathrm{~km}$ al E de Chancah Veracruz, 4. III.1985, E. Gutiérrez 509 (MEXU); km 21 de Felipe Carrillo Puerto a Vigía Chico, 19.Il.1991, C. Salazar 57 (MEXU); Xkomhá, 26.V.1985, E. Ukán 4055 (MEXU); km 1.5 carretera crucero de Vigía Chico, 26.IX.1985, R. Villanueva 455 (MEXU). Municipio José María Morelos, $0.8 \mathrm{~km}$ al O de Cafetalito camino El Cafeta - Kankabchén, 12.III.2004, D. Álvarez et al. 8051 (MEXU); $4.15 \mathrm{~km}$ al E del poblado Plan de la Noria, 16.III.2004, D. Álvarez y A. Ramírez 8213 (MEXU); $2.78 \mathrm{~km}$ al $\mathrm{N}$ del poblado Zafarrancho, 19.III.2004, D. Álvarez y A. Ramírez 8481 (MEXU). Municipio Lázaro Cárdenas, brecha al antiguo aeropuerto de Cancún, 22.II.1981, E. Cabrera y G. Ibarra 1437 (MEXU); antiguo aeropuerto de Cancún, 16.III.1981, E. Cabrera y S. Zárate 1517 (MEXU); ejido Tres Garantías, 21.IX.1983, C. Chan 2999 (MEXU); Yucatan Peninsula, Yalahau Region, El Edén Ecological Reserve, ca. $30 \mathrm{mi}(45 \mathrm{~km}) \mathrm{NW}$ of Cancún, around Cenote Ayim, 7. IV.1999, R. A. Palestina 703 (MO). Municipio Othón P. Blan- 
co, $4.5 \mathrm{~km}$ al S de Xcalak, 23.X.1990, E. Cabrera 17076 (MEXU); $56 \mathrm{~km}$ E of hwy 307 on road to Majahual, at Majahual, 8.VIII.1989, L. R. Landrum y S. Landrum 6472 (ASU); aeropuerto de Chetumal, 6.IX.2008, V. Peña s.n. (MEXU). Municipio Solidaridad, $8 \mathrm{~km}$ al $\mathrm{S}$ de la desviación a Puerto Morelos sobre la carretera a Tulum, en el rancho El Chechen, 12.III.1985, E. Cabrera y H. de Cabrera 7802 (MEXU, MO); ruinas de Cobá, 15.V.1981, J. S. Flores y E. Ucán 8449 (MEXU); Cobá, camino a la Pirámide llamada Iglesia, 19. II.1976, P. Moreno 468 (MEXU); 7 km al SO de Puerto Morelos, en la carretera a Playa del Carmen, 1.II.1980, M. Sousa et al. 11054 (MEXU); Cobá, zona arqueológica, 9.IX.1980, E. Ucán 375 (MEXU). Municipio Tulum, sea cliffs behind the beach by the ruins at Tulum, 13.III.1997, R. M. King y R. M. Garvey 10658 (MO); Tulum, rumbo a las cabañas, 13. IV.1987, E. Ucán y M. Poot 5106 (MEXU). San Luis Potosí, municipio El Naranjo, near the waterfall at El Santo, 20. II.1961, R. M. King y G. Diboll 3869 (F). Municipio Xilitla, mountains along gravel road to Jalpan, $2 \mathrm{mi} \mathrm{W}$ of Xilitla, 25.III.1961, R. M. King y R. Johnson 4228 (F). Sinaloa, municipio Ahome, $2 \mathrm{mi}$ N of jct with Sinaloa rte. 32 along Mex 15, NW of Los Mochis, 1.VI.1974, M. L. Roberts 10152 (ASU). Municipio Culiacán, Imala, 19.III.1940, W. S. Gentry 5931 (MO); Península de Lucenilla, frente a Altata, 15.III.1985, P. Tenorio et al. 8344 (IEB). Municipio Mazatlán, valley near Mazatlán, northward, 13.VI.1849, J. Gregg 1203 (MO). Sonora, municipio Álamos, Río Mayo Region. $0.8 \mathrm{~km} \mathrm{~N}$ of Arroyo el Mentidero on El Chinal road, $10.5 \mathrm{~km}$ S of Álamos, 15.III.1994, T. R. Van Devender 178 (ASU). Municipio Huatabampo, Yavaros, 4.IV.1994, A. C. Sanders et al. 14322 (MO). Tabasco, municipio Huimanguillo, camino a la antena de microondas del cerro de Las Flores, 28.III.1996, M. A. Guadarrama et al. s.n. (MEXU). Municipio Tenosique, sobre la carretera 20, $3 \mathrm{~km}$ al O de Tenosique, 11.III.1976, J. I. Calzada y J. Arellano 2220 (F, MEXU); cerro al S del campamento La Escollera, 19.II.1983, S. Zamudio 748 (MEXU). Tamaulipas, municipio Altamira, 2 mi NE of Altamira, 3.III.1961, $R$. M. King y R. Johnson 4068 (F, NY). Municipio Casas, Arroyo El Sarnoso, $8 \mathrm{~km}$ al O de Villa de Casas, 8.IV.1998, A. Mora y M. G. Aguilar 6884 (MEXU). Municipio Ciudad Victoria, along route 70, $3 \mathrm{mi}$ S of Ciudad Victoria, 6.IV.1961, R. M. King y R. Johnson 4501 (F); vicinity of Victoria, 1.II.1907, E. Palmer 1 (F, MO). Municipio Llera, Sierra Madre Oriental, ca. 6-8 km NW of El Encino, ca. $30 \mathrm{~km} \mathrm{~N}$ of HW 85 of Ciudad Mante, 2.IV.1980, J. Pruski y N. Barker 1695 (MO). Municipio Ocampo, on the road to Tula, 5-6 mi NW of Ocampo, 25.II.1961, R. M. King y G. Diboll 3927 (F, NY). Municipio Soto La Marina, Río Soto La Marina, en el puente a las afueras de la ciudad, 8.IV.1998, A. Mora y M. G. Aguilar 6889 (MEXU). Municipio Tampico, Tampico, 1.I.1910, E. Palmer 75 (F, MO). Municipio Xicoténcatl, Rancho Calabazas, on the Río Sabinas, across from the village of Azteca, $3 \mathrm{mi} \mathrm{W}$ of Federal route 85, at point about $20 \mathrm{mi}$ N of Ciudad Mante, 30.III.1975, N. A. Harriman et al. 10965 (F). Veracruz, Municipio Actopan, ladera $\mathrm{N}$ del cerro de Los Metates, 25. VI.1972, J. Dorantes 1012 (F, MEXU); Laguna Farallón, 13. III.1973, A. Lot 1272 (F); Estación Biológica Morro de La Mancha, 13.V.1981, P. Moreno et al. 24 (MEXU). Municipio Alto Lucero de Gutiérrez Barrios, Laguna Verde, III.1941, Anónimo 8979 (MO); ejido de Santa Ana, 18.IV.1970, E. Jarquin y A. Lot 20 (MEXU). Municipio Atoyac, Atoyac, 30.I.1940, C. Conzatti y C. Conzatti 6004 (MEXU). Municipio Atzalan, El Filo, 30.III.1984, E. Ventura 1981 (ENCB, IEB, MO). Municipio Catemaco, lado E de Zapoapan de Cabañas, 11.II.1972, J. H. Beaman 5649 (MEXU). Municipio Cazones de Herrera, Rancho Nuevo, 2.III.1982, V. Cortés 180 (MEXU); La Encantada, 5.IV.2011, G. Romo 1367 (MEXU). Municipio Chicontepec, Pichol $1 \mathrm{~km}$ al N, 21.III.1988, C. Durán 209 (IEB, MEXU). Municipio Chocamán, $2.5 \mathrm{~km} \mathrm{~S}$ of Chocamán, 17.I.1975, G. K. Arp 4547 (F). Municipio Coatepec, Xalapa to Huatusco via Coatepec, Tuzamapan and Tlaltetela, roadside, 1.111 .2008$, T. B. Croat 100070 (MO); $1.5 \mathrm{~km}$ $W$ of Cosamaloapan, on road to dam at Cinco Palos, $1 \mathrm{~km} \mathrm{~N}$ of Coatepec, 24.IV.1983, M. Nee y K. Taylor 26742 (F, MO). Municipio Coxquihui, Cerro Akgmuxni al O de Chapultepec, 10.IV.1985, P. Tenorio et al. 8554 (F, MEXU). Municipio Emiliano Zapata, ejido González Ortega, 2.III.1980, S. Avendaño et al. 603 (F), camino a El Lencero, casi entronque con exhacienda, 23.II.1983, M. Lascurain 106 (MEXU); camino a Lencero, 23.II.1986, A. Ruiz 31 (MEXU). Municipio Hueyapan de Ocampo, Santa Rosa Cintepec, entre la carretera Catemaco - Coatzacoalcos, 16.III.1985, R. Cedillo 3104 (MEXU); Santa Rosa, Loma Larga, 14.XI.1999, M. Leonti 153 (MEXU). Municipio Jalacingo, Bravo Grande, 4.III.1982, F. Ventura 18464 (ENCB, IEB, MEXU). Municipio Maltrata, Cumbres de Maltrata, 26.III.1970, R. Hernández 655 (F, MEXU). Munici- 
pio Misantla, $1.65 \mathrm{~km}$ al SSE de Pueblo Viejo, camino a Manuel Gutiérrez Nájera, 3.III.2012, E. Martínez S. y M. Mora 42714 (MEXU); 2 km al E de Misantla, rancho particular, 8. IV.2002, A. Rincón et al. 2932 (MEXU). Municipio Pánuco, El Mirador, presa Paso de Piedras, 13.VII.1978, J. I. Calzada y W. Márquez 4566 (F); loc. cit., 13.VII.1978, J. I. Calzada y W. Márquez 4579 (F); foodplain of Pánuco river, near Ébano, 28.II.1939, H. LeSeur 469 (F). Municipio Puente Nacional, Puente Nacional, carretera Xalapa - Veracruz, Emiliano Zapata, 10.IV.1972, J. Dorantes 523 (F, MEXU). Municipio Tecolutla, Paso del Río, Río Riachuelos, 16.X.1982, Brigada de dunas (Facultad de Ciencias) 915 (IEB); desviación NautlaTecolutla, 3.II.1965, A. Gómez 50 (MEXU). Municipio Tihuatlán, Tihuatlán, 19.V.1969, H. Puig 4643 (MEXU). Municipio Tlaltetela, El Limón, 21.II.1978, F. Ventura 15021 (ENCB, MO); La Mesa, 27.II.1978, F. Ventura 15027 (ENCB, MEXU). Municipio Tuxpan, along route $122,3 \mathrm{mi} \mathrm{W}$ of Tuxpan, 3.III.1961, R. M. King y R. Johnson 4127 (F). Municipio Úrsulo Galván, NO de Úrsulo Galván, a orilla del Río Actopan, 21.I.1990, L. Orea 273 (MEXU). Municipio Xalapa, Instalaciones de la SAGAR, 21.VI.2000, J. Lizama 1559 (MEXU), Cañada Tembladeras, 22.XII.1989, P. Zamora 1829 (IEB, MEXU). Municipio Yecuatla, El Cajón, 13.III.1972, F. Ventura 5056 (ASU, ENCB, UNM). Yucatán, municipio Cantamayec, Jardín de plantas medicinales a tres cuadras del centro de Cantamayec, 24.III.2011, M. Méndes et al. 1977 (MEXU). Municipio Celestún, $10 \mathrm{~km}$ antes de Celestún, 22.IV.1983, C. Chan 2200 (MEXU); sobre la carretera Umán - Celestún, $15 \mathrm{~km}$ antes de Celestún, 3.V.1983, E. Góngora 338 (MEXU); $5 \mathrm{~km}$ desviación a Chunchucmil, carretera Kinchil - Celestún, 27.III.1985, E. Rivera 283 (MEXU). Municipio Cenotillo, 11.7 km al NE de Cenotillo, 2.III.1988, L. Santos 89 (MEXU). Municipio Dzemul, cerca de las ruinas de Xcambó, 8.II.2008, J. P. Pinzón et al. 11 (MEXU). Municipio Dzilam de Bravo, N del rancho San Pedro III, reserva ecológica de Dzilam, 16. VI.1991, J. C. Tun 256 (MEXU). Municipio Dzidzantun, 500 $\mathrm{m}$ al S de Santa Clara, camino a Chabilhao, 24.VII.1987, E. Cabrera y H. de Cabrera 13730 (MEXU, MO). Municipio Espita, 4 km al E de Espita, 15.III.1990, E. Reyes 49 (MEXU); 7 km al O de Espita, 30.I.1992, E. Reyes 130 (MEXU). Municipio Izamal, Izamal, 1895, G. F. Gaumer 399, (MO, NY); Izamal, 22.II.1906, J. M. Greenman 455 (F). Municipio Kanasín, alrededor del poblado San Antonio Tehuitz, 5.X.1984, C.
Chan 4422 (MEXU). Municipio Kantunil, $15.5 \mathrm{~km}$ al E de Kantunil, luego del inicio de la autopista Mérida - Valladolid, 25.III.1999, G. Carnevali et al. 5375 (F, MEXU, MO). Municipio Kinchil, $3 \mathrm{~km}$ al $\mathrm{O}$ de la desviación a Chunchucmil, carretera a Celestún, 6.V.1993, R. Durán 1744 (HCIB, MEXU, $\mathrm{MO})$. Municipio Mérida, fraccionamiento Jardines de Mérida, 1.III.1981, J. S. Flores 8054 (MEXU). Municipio Muna, Uxmal, 20.IV.1973, A. F. Clewell 3835(MO). Municipio Progreso, km 10-16 carretera Sierra Papacal - Chuburná - Puerto Progreso, 28.III.1992, G. Campos y P. Simá 2840 (MEXU); $8 \mathrm{~km}$ al S de Progreso, 13.III.1997, F. Kú 655 (MEXU); $6 \mathrm{~km}$ al S de Chuburná, 30.XI.1994, E. Reyes 421 (MEXU). Municipio Río Lagartos, entronque del camino Río Lagartos, rumbo a Las Coloradas, 13.III.1985, C. Chan 4783 (MEXU); 1 km al O del entronque de Río Lagartos, rumbo a San Felipe, 9. II.1992, R. Durán 1538 (MEXU). Municipio Sotuta, Tabi, N side of the church inside the fence, 25.VII.2007, A. Hopkins 42 (MEXU); km 6 camino Tixacacaltuyub - Sotuta, 28.I.1983, P. Simá 59 (MEXU). Municipio Tekax, $30 \mathrm{~km} \mathrm{~S}$ of Xul, 29. IX.1982, S. P. Darwin 2351 (F, MEXU). Municipio Tinum, Jardín Botánico Balancaché zona D, 21.I.1989, S. Escalante 475 (MEXU); loc. cit., 18.II.1989, S. Escalante 483 (MEXU). Municipio Tixcacalcupul, comunity Ekpedz, 20.VI.1996, A. Ankli 9 (MEXU). Municipio Tizimín, $23 \mathrm{~km}$ al $\mathrm{N}$ de Kantunilkin, sobre el camino a Colonia Yucatán, 23.VII.1987, E. Cabrera y H. de Cabrera 13699 (MEXU); 6-8 km al N de Colonia Yucatán, camino a El Cuyo, 22.III.1988, E. Cabrera y H. de Cabrera 15716 (MEXU, MO). Municipio Tzucacab, roadway from Dzuiche, 17.I.1967, A. P. Covich 6722 (MEXU). Municipio Yaxcabá, Tixcacaltuyub, III.1988, V. Rico 733 (MEXU); 5 km rumbo a Peto, 14.I.1987, P. Simá 296 (MEXU); Tixcacaltuyub $2 \mathrm{~km}$ rumbo a Sotuta, lado izquierdo del camino, 11.II.1988, P. Simá 495 (MEXU); Tixcacaltuyub, Rancho Ekbalam, 23. XII.1980, C. Vargas 317 (MEXU); Tixcacaltuyub, $2 \mathrm{~km}$ al N, 17.XI.1981, C. Vargas 347 (MEXU).

Pluchea mexicana (Goodfrey) G.L. Nesom, Phytologia 67(2): 159. 1989.

इ Pluchea rosea var. mexicana R.K. Goodfrey, J. Elisha Mitchell Sci. Soc. 68(2): 269. 1952. TIPO: MÉXICO. San Luis Potosí, by streams, alkaline plains, Hacienda de Angostura. 5.VIII.1891, C. G. Pringle 3813 (holotipo: GH-00011330!; iso- 
tipos: BR-0000005429851!, F-0051022!, MICH-1107629!, MU-000021372!, NY-00232536!, PH-00030711!, PH00030712!, PUL-00000150!, TEX-00000386!, UC-35676!, UC-88368!, US-00129412!).

Hierbas perennes, 0.6-1.2 $\mathrm{m}$ de alto; tallos sin alas; ramas pilosas y glandulares; hojas sésiles, láminas lanceoladas u oblongo-lanceoladas, $3.5-4.8 \times 1.2-2.1 \mathrm{~cm}$, ápice agudo, base auriculada, margen dentado, pilosas y glandulares en ambas superficies; inflorescencias cimosas con (12-)40-70 cabezuelas, pedúnculos individuales $2-5.8 \mathrm{~mm}$ de largo, pilosos y glandulares; involucro campanulado 5.8 7.3 ×6.7-8.2 mm; filarios (24-)28-36, 3-4 seriados, subiguales, elípticos o lanceolados, ápice agudo, pilosos y glandulares; flores periféricas 110-130, corolas (3-)4-4.2 mm de largo, cipselas 0.6-0.7 mm de largo, pardas, pilosas; vilano con 18-20 cerdas capilares, 3.1-4.3 mm de largo; flores del disco 25-30, corolas (3.8-)4.6-5 mm de largo, rosadas, lóbulos triangular-lanceolados, glandulares en el ápice; anteras 2.1-2.4 mm de largo; estilo 4.6-5.3 $\mathrm{mm}$ de largo; cipselas 0.9-1.2 mm de largo, pardas, pilosas y glandulares; vilano con $16-20$ cerdas capilares, 3.8-4.7 mm de largo.

Comentarios taxonómicos: Pluchea mexicana se elevó a rango de especie a partir de $P$. rosea var. mexicana, descrita por Goodfrey (1952), para nombrar especímenes afines a $P$. rosea, pero con distribución restringida a la región de Río Verde, San Luis Potosí, donde predominan suelos gipsófilos alcalinos que propician un elevado nivel de endemismos de Asteraceae (Nesom, 1989). Pluchea mexicana difiere de $P$. baccharis por la forma del ápice de las hojas (agudo vs. apiculado), el tipo de glándulas foliares (estipitadas vs. sésiles), indumento en los tallos (piloso y glandular vs. tomentuloso); el número de cabezuelas en las inflorescencias ( $\geq 12$ vs. $\leq 10$ ), además de la distribución geográfica.

Distribución: Pluchea mexicana es endémica de San Luis Potosí, en elevaciones de 570-1010 m s.n.m. (Fig. 3).

Hábitat: vegetación hidrófila; en suelos alcalinos.

Fenología: florece y fructifica de junio a agosto.
Ejemplares examinados: MÉXICO. San Luis Potosí, municipio Río Verde, at Media Luna, near Rio Verde, 2.VI.1904, E. Palmer 75 (F, NY). Municipio Xilitla, minas de San Rafael, plains between Tiburcio and Angostura, VII.1911, C. A. Purpus 5128 (F).

Pluchea odorata (L.) Cass., Dict. Sci. Nat. (ed. 2) 42: 3. 1826.

इ Conyza odorata L., Syst. Nat. (ed. 10) 2: 1213. 1759. TIPO: JAMAICA. Jamaica, s.f., B. L. Sloane s.n. (lectotipo: BM000589029, designado por Britten, 1898).

三 Conyza purpurascens Sw., Prodr. 112. 1788., nom. illeg.

= Conyza marilandica Mich., Fl. Bor.-Amer. 2: 126. 1803. TIPO: ESTADOS UNIDOS DE AMÉRICA. Hab. a Pensylvania and Carolinam, (lectotipo: lámina 104, Hortus Dillenius, designado por Nesom, 2004).

三 Gymnostyles marilandica (Mich.) Raf., Amer. Monthly Mag. \& Crit. Rev. 2(4): 268. 1818.

इ Pluchea marilandica (Mich.) Cass., Dict. Sci. Nat. (ed. 2) 42: 2. 1826.

= Pluchea petiolata Cass., Dict. Sci. Nat. (ed. 2) 42: 2. 1826. TIPO: No localizado.

= Conyza angustifolia Nutt., J. Acad. Nat. Sci. Philadelphia 7(1): 109. 1834. TIPO: ESTADOS UNIDOS DE AMÉRICA. South Carolina, from whence it was received by Mr. Durand. (holotipo: no localizado).

= Pluchea glabrata DC., Prodr. 5: 452. 1836. TIPO: ANTILLAS. Guadaloupe, Pointe-à-Pitre, 17.VI.1824, G. S. Perrottet s.n. (sintipo: G-00457997!).

इ Pluchea camphorata (L.) DC. var. angustifolia (Nutt.) Torr. \& A. Gray, FI. N. Amer. 2: 261. 1842.

इ Pluchea purpurascens var. glabrata (DC.) Griseb., Cat. PI. Cub. 150. 1866.

इ Placus odoratus (L.) M. Gómez, Anales Soc. Esp. Hist. Nat., 19(2): 273.1890.

इ Placus purpurascens (Sw.) M. Gómez, Anales Soc. Esp. Hist. Nat., 19(2): 273. 1890.

三 Placus purpurascens var. glabratum M. Gómez, Anales Soc. Esp. Hist. Nat., 19(2): 273. 1890.

= Pluchea purpurascens fo. obovata Fernald, Rhodora 44(528): 476. 1942. TIPO: ESTADOS UNIDOS DE AMÉRICA. Virginia, James City County. Tidal marsh along Powhatan Creek, N of Jamestown Island, 22.VIII.1939, M. L. Fernald y 
B. Long 11191 (holotipo: GH-00011324!).

= Pluchea odorata var. succulenta (Fernald) Cronquist, Vasc. FI. S.E.U.S. 1: 175. 1980. TIPO: ESTADOS UNIDOS DE AMÉRICA. Massachusetts, Boston, ditch in old marsh Black Bay, 17.IX.1910, E. F. Williams s.n. (holotipo: GH00011325!).

Hierbas anuales, $0.25-1.5(-1.8) \mathrm{m}$ de alto; tallos $\sin$ alas; ramas pilosas; hojas con peciolos $0.9-3.2 \mathrm{~cm}$ de largo, láminas elípticas, rara vez lanceoladas u ovadas, 4.5-12.8 $\mathrm{cm}$ de largo, $1.5-5 \mathrm{~cm}$ de ancho, ápice agudo, base cuneada, margen serrado, pilosas en ambas superficies, rara vez con glándulas; inflorescencias corimbiformes con 18-690 cabezuelas, pedúnculos individuales $0.3-1.2 \mathrm{~cm}$ de largo, pilosos; involucro campanulado (4-)4.6-4 mm de largo, 6.8-9.7 mm de ancho; filarios (20-)24-30(-36), 3-4 seriados, imbricados, elípticos, ápice agudo, pilosos y glandulares; flores periféricas 340-380, corolas 3-4 mm de largo; cipselas 0.6-0.87 mm de largo, pardas o negras, pilosas; vilano con $15-18$ cerdas capilares, 3.4-4 mm de largo; flores del disco 12-15, corolas (3-)3.6-5 mm de largo, rosadas, lóbulos lanceolados, glandulares y papilosos en el ápice; anteras (1.4-)1.6-2 mm de largo; estilo (3.2-)4.2-5.3 mm de largo; cipselas (0.6-)0.8-1.2 mm de largo, pardas o negras, pilosas; vilano con $18-20$ cerdas capilares, (2.6-)3.5-4.2 mm de largo. (Figs. 1C, 6).

Comentarios taxonómicos: Pluchea odorata se ha confundido con P. carolinensis, debido a que Goodfrey (1952) la consideró como su sinónimo. No obstante, ambos taxones presentan diferencias morfológicas conspicuas entre las que destacan: hábito de crecimiento (hierbas anuales vs. arbustos), indumento del tallo (glabrescente vs. piloso); margen de las hojas (dentado vs. entero), número de flores periféricas (340-380 vs. 148-180) y discoides (15-18 vs. 28-40) (Figs. 4, 6).

Distribución: en México, Pluchea odorata se encuentra en Baja California, Campeche, Chiapas, Coahuila, Colima, Guerrero, Nayarit, Nuevo León, Oaxaca, Quintana Roo, San Luis Potosí, Sinaloa, Sonora, Tabasco, Tamaulipas, Veracruz y Yucatán, en elevaciones de 0-1000 m s.n.m. (Fig. 7).
Hábitat: manglares, matorrales xerófilos, sabanas, selvas altas perennifolias, bajas caducifolias, vegetación de dunas costeras, hidrófila, secundaria y ecotonos. En zonas inundables temporales o permanentes, orilla de arroyos, canales, lagunas, pantanos o ríos con suelos arenosos, arcillosos, aluviales, limosos, litosoles o regosoles.

Fenología: florece y fructifica durante todo el año.

Ejemplares examinados: MÉXICO. Baja California, municipio Ensenada, San Telmo river valley, E of San Telmo village, 29.VIII.1978, R. Noyes et al. 718 (UCR); Todos Santos Bay, 22.IX.1884, C. R. Orcutt 1236 (F); La Bocana de Santo Tomas, on Punto Chino road, 20.IX.1980, R. Moran 29249 (DES, MO); lagoon at the mouth of the Río del Rosario, 10.X.1987, A. C. Sanders 7401 (UCR); flats near San Telmo, 26.IX.1930, I. L. Wigging y D. Demaree 5056 (F). Municipio Mexicali, stream banks near Rancho San Jacinto, $45 \mathrm{mi} S$ of Ensenada, 7.IX.1930, I. L. Wigging y D. Demaree 4735 (F); salt lagoon near the sea, $5 \mathrm{mi}$ W of Hamilton Ranch, 9. IX.1930, I. L. Wigging y D. Demaree 4765 (F). Municipio Tecate, Kumeyaay Rancho of Ha-a, ca. 25 km SE of Tecate, 10.X.1976, R. Moran 23978 (MO). Campeche, municipio Calkini, $4 \mathrm{~km}$ al $\mathrm{O}$ de Tunkasche, sobre el camino Calkini Punta Arenas, 25.III.1988, E. Cabrera y H. de Cabrera 15846 (IEB, MEXU); Isla Punta Arenas, 17.III.1983, C. Chan 1936 (MEXU); loc. cit., 22.V.1981, C. Chan et al. 417 (MEXU); 1 km antes de llegar a El Remate, camino de Tankuché, 17.X.1984, C. Chan 4290 (MEXU); 3.5 km después del Remate - Punta Arena, 27.VII.1981, C. Chan y M. Burgos 661 (MEXU); 7 km al O del Remate camino a Punta Arenas, 22.VIII.1985, R. Durán y M. Ek 1322 (MEXU); camino de El Remate, viniendo de Isla Punta Arenas, 17.III.1983, J. S. Flores 9764 (MEXU); km 1 Tankuché - Punta Arena, 3.V.1985, M. Narváez 1370 (MEXU); $5 \mathrm{~km}$ al O de El Remate, 16.IV.1981, H. Quero y $R$. Grether 3044 (MEXU); 11 km al O de Tankuché, carretera a Isla Arena, 28.XI.1993, F. Tun et al. 160 (MEXU). Municipio Campeche, Unidad de Evaluación y Monitoreo de la Biodiversidad de Hampolol, 20.VI.1996, P. Zamora y D. Méndez 5114 (MEXU). Municipio Carmen, Punta Zacatal - Puerto Rico, 23.III.1985, C. Chan 4915 (MEXU); predio ganadero al E del terreno de la UAIM, ejido Los Manantiales, 18.VII.1994, 


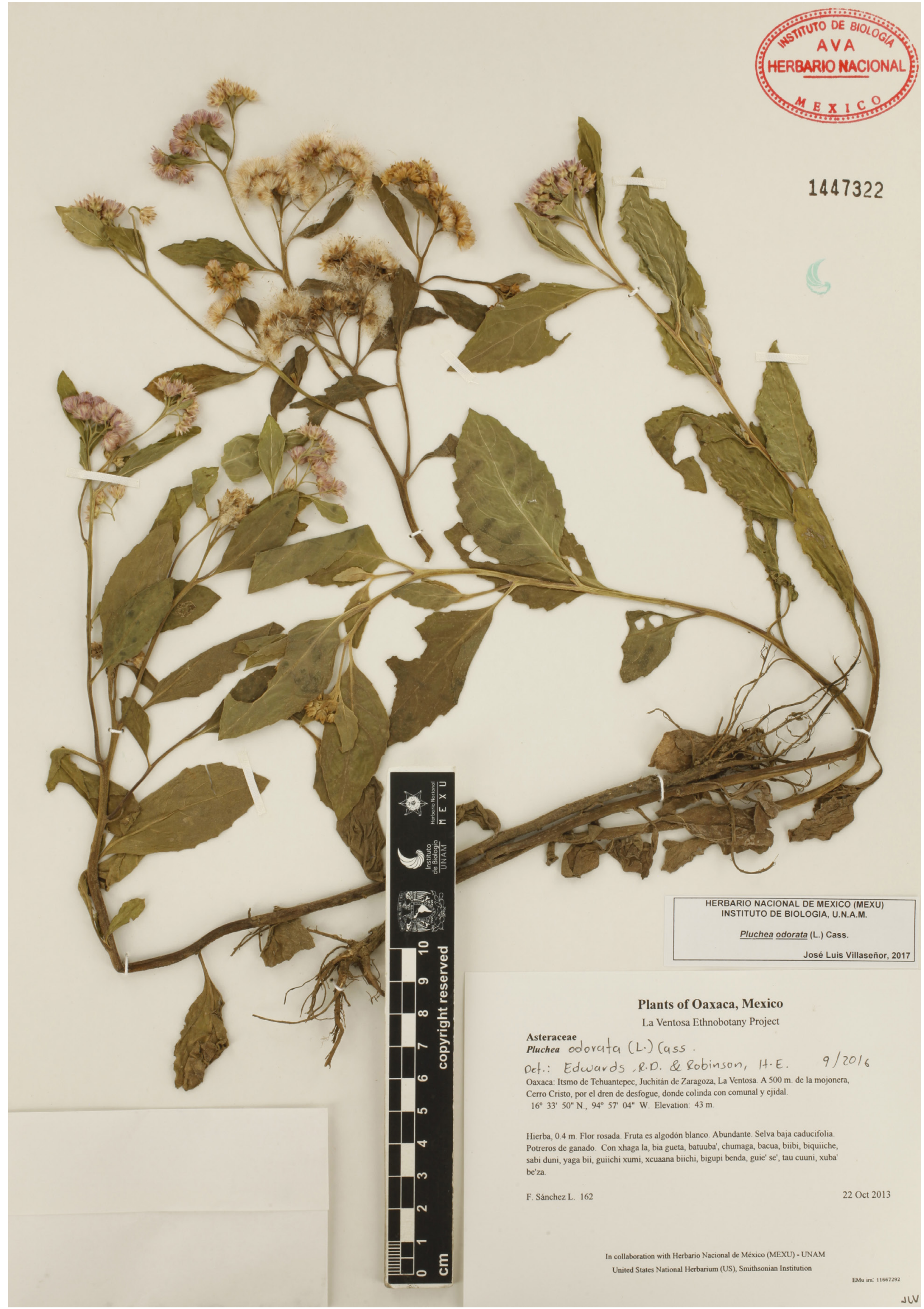

Figura 6: Ejemplar herborizado de Pluchea odorata (L.) Cass. 


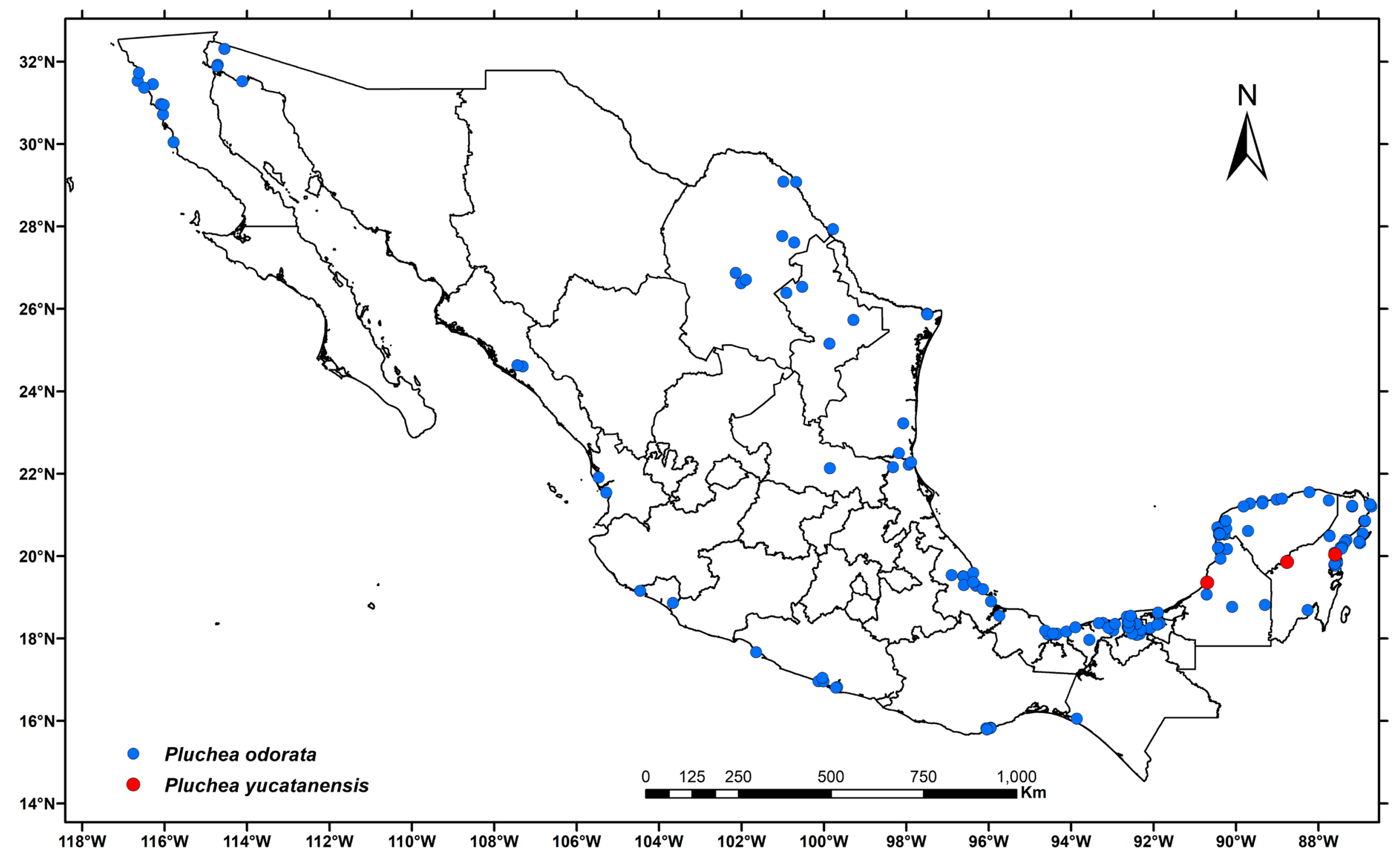


Juárez 257 (MEXU); Laguna El Vapor, 22.VI.1988, D. Ocaña 216 (MEXU). Municipio Champotón, predio rústico margen derecho, carretera Escárcega - Champotón tramo Xbacab El Pocito, 17.XI.1999, E. Osorno 1053 (MEXU). Municipio Hecelchakán: $35 \mathrm{~km}$ al E de Dzodzil sobre el camino Pomuch - Isla Jaina, 27.VII.1987, E. Cabrera y H. de Cabrera 13995 (MEXU, MO); 16 km al O de Hecelchacán, 3.X.1986, E. Cabrera et al. 11981 (MEXU); camino de Pomuch - Isla de Jaina, 27.V.1983, E. Góngora y G. S. Flores 543 (MEXU). Municipio Hopelchén, $3 \mathrm{~km}$ al $O$ de Unión 20 de junio (Mancolona), 2.VIII.1997, E. Martínez S. et al. 27964 (MEXU). Municipio Palizada, Palizada, 25.VII.1939, E. Matuda 3859 (F, MEXU); Río Palizada, 3.IX.1988, D. Ocaña y C. Coronado 268 (MEXU). Chiapas, municipio Tonalá, Paredón, Tonalá, 14.I.1946, E. Matuda 16278 (MEXU, MO). Coahuila, municipio Cuatro Ciénegas, Poza de La Becerra en Cuatro Ciénegas, 26.X.1985, J. A. Villarreal et al. 3198 (MEXU); Cuatro Ciénegas Basin, Los Fresnos Laguna, 28. VIII.1967, D. J. Pinkava et al. 4298 (ASU). Municipio Jiménez, Río San Diego, carretera 57, 13.VIII.2001, M. A. Carranza e I. Ramírez 3476 (IEB, MEXU); ejido Cristales, lado $\mathrm{N}$ del Río San Diego, 7.X.2007, J. A. Encina et al. 1894 (IEB). Municipio Nadadores, 25 air mi WNW of Monclova, $2 \mathrm{mi} \mathrm{W}$ of Nadadores km 32 along Mexican highway 30, 9.X.1976, J. Henrickson y M. Dillon 15570 (MEXU, NMC). Municipio Sabinas, Río Sabinas, parque Guadalupe Victoria, ejido Pueblo Nuevo, al S de Sabinas, 8.X.2005, J. A. Encina y A. Zárate 1826 (IEB); Juárez, $100 \mathrm{mi} \mathrm{N}$ of Monclova, on the Sabinas river, 23.IX.1880, E. Palmer 544 (F). Colima, municipio Manzanillo, W side of bay across from Manzanillo, about $5 \mathrm{mi}$ WNW of city, VII.1956, D. P. Gregory y G. Eiten 314 (MEXU, MO). Municipio Tecomán, $10 \mathrm{~km}$ de Chanchopa en la desviación de San Miguel, 26.IV.1990, M. L. Román 1309 (MEXU). Guerrero, municipio Acapulco, Laguna Tres Palos, Acapulco, 20.IX.1968, W. L. Boege 822 (MEXU); E of Acapulco on highway 200 about $6 \mathrm{mi} E$ of junction with airport road, 10.II.1971, J. Freeland y L. Spetzman 213 (MEXU). Municipio Coyuca de Benítez, El Embarcadero, 10.VI.1986, A. Aquino 19 (MEXU); Mangle Seco, aprox. $5 \mathrm{~km}$ al S de Salinas, 8.V.1986, R. M. Fonseca 146 (MEXU); El Bordonal, 13.I.1987, S. Gama 516 (FCME, IEB); manglar El Carrizal, 12.VII.1981, M. Ojeda 73 (MEXU). Municipio José Azueta, entre La Punta Ixtapa y el cerro El Rialito, 28.V.1991, G. Cas- tillo et al. 6569 (MEXU). Nayarit, municipio Mexcaltitlán, s.f., J. González 5538 (MEXU). Municipio San Blas, behind the beach at San Blas, 11.VIII.1960, R. M. King 3697 (MEXU). Nuevo León, municipio Bustamante, Cañón de Bustamante, Arroyo Huizache along the Sabinas River, $3.9 \mathrm{~km}$ W of Bustamante, 16.X.1992, L. A. Prathery A. Hempel 1289 (MEXU). Municipio China, $7.8 \mathrm{~km}$ E of turnoff to Los Herreras on Mex 40, near Presa of San Juan, 18.IX.2001, R. A. Bye 28333 (MEXU). Municipio Mina, El Manantial, área cercana al ejido La Soledad, carretera Monterrey - Monclova, 22. VIII.1984, M. A. Carranza et al. 2638 (MEXU). Municipio Montemorelos, cuenca del Río Pilón, 2 km al SW de El Chapotal, 13.X.1983, R. Sánchez 420 (MEXU). Oaxaca, municipio Santa María Huatulco, boca del Río Copalita, 11.XI.1992, G. Castillo y P. Zamora 9749 (MEXU); Barra de Copalita, Bahía de Huatulco, 9.I.1991, M. Illescas 4 (MEXU). Municipio Santiago Astata, Barra de la Cruz, 2 km SSE, laguna en la playa, 26.XI.1998, M. Elorsa 1161 (MEXU); laguna El Cerrito, $3 \mathrm{~km}$ al SE de Barra de Cruz, 13.II.2001, M. Elorsa 4255 (MEXU); La Laguna, $2 \mathrm{~km}$ al $\mathrm{S}$ de Barra de La Cruz donde desemboca el Río Chacalapa, 13.III.2002, M. Elorsa 5861 (IEB, MEXU). Quintana Roo, municipio Benito Juárez, carretera de entrada a Puerto Morelos, a partir de la carretera Cancún - Tulum, 22.III.1976, P. Moreno 558 (MEXU); 1 km al $\mathrm{N}$ de Puerto Morelos sobre la carretera Cancún - Tulum, 21.VI.1976, P. Moreno 816 (MEXU); carretera Cancún Puerto Juárez, 1.VII.19080, A. Puch 23 (MEXU). Municipio Cozumel, $10 \mathrm{~km}$ al $\mathrm{S}$ de Akumal, sobre la carretera Cancún - Tulum, 2.VI.1983, E. Cabrera y H. de Cabrera 4754 (MEXU); sobre el camino a Palancar, $4 \mathrm{~km}$ al $\mathrm{S}$ de la desviación a El Cedral, Isla de Cozumel, 24.IV.1987, E. Cabrera y H. de Cabrera 13111 (MEXU, MO); sobre el camino a Palancar, 6 km al SW de la desviación a El Cedral, Isla de Cozumel, 14.I.1987, E. Cabrera y H. de Cabrera 13022 (MEXU); sobre el camino a Palancar, $22 \mathrm{~km}$ al S de San Miguel de Cozumel, Isla de Cozumel, 18.VI.1987, E. Cabrera y H. de Cabrera 13609 (MEXU, MO); brecha en dirección NE-NO en la punta NO de la Isla Cozumel, a $5.6 \mathrm{~km}$ de la Ciudad de San Miguel Cozumel, 2.V.1985, J. López-Portillo y A. López-Portillo 6 (MEXU). Municipio Felipe Carrillo Puerto, Ramonal, al SE de Punta Allen, 30.VIII.1982, E. Cabrera y H. de Cabrera 3439 (MEXU); $21 \mathrm{~km}$ al $\mathrm{S}$ de Tulum, a la orilla de la Laguna de Muyil, 19.V.1982, E. Cabrera et al. 2772 (MEXU); Laguna Mujil, 1 
$\mathrm{km} \mathrm{E}$ of Ruinas Chunyaxché and $26 \mathrm{~km} \mathrm{SW}$ of Tulum, 19.V.1982, G. Davidse et al. 20631 (MEXU, MO); 3 km al N del Crucero de Vigía Chico, 3.I.1984, R. Durán e I. Olmsted 704 (MEXU); $1 \mathrm{~km}$ al E del rancho El Yuras, 17.IX.1985, I. Olmsted y M. A. Marmolejo 42 (MEXU); Vigía Chico, 7. VII.1980, A. Puch 40 (MEXU); km 4 carretera Vigía ChicoFelipe Carrillo Puerto, 27.V.1985, R. Villanueva 346 (MEXU); km 3 carretera Vigía Chico - Felipe Carrillo Puerto, 5.II.1986, R. Villanueva 618 (MEXU). Municipio Isla Mujeres, Isla Mujeres, parte S, 5.VIII.1983, C. Chan 2710 (MEXU); Isla Mujeres, Puerto de Abrigo, 13.V.1981, J. S. Flores y E. Ucán 8425 (MEXU); zona de manglares, atrás de la empacadora Romeo, 14.V.1981, E. Ucán y J. S. Flores 1061 (MEXU); Isla Mujeres, near S end of Laguna Makax, 3.VIII.1979, G. L. Webster y S. Lynch 17631 (MEXU). Municipio José María Morelos, Laguna de Chichancanab, s.f., G. F. Gaumer 2175 (F); loc. cit., s.f., G. F. Gaumer 2453 (MO). Municipio Lázaro Cárdenas, El Edén Reserve, ca. 30 mi NW of Cancún, area surrounding Laguna Chamacos, approximately $1 \mathrm{~km} \mathrm{SW}$ of station building, 9.IV.1999, R. Palestina 724 (UCR); El Edén Reserve, 30 mi NW of Cancún, 8.VII.1997, G. P. Schultz et al. 589 (UCR); El Edén Reserve, ca. 30 mi NW of Cancun, area surrounding Laguna Chamacos, approximately $1 \mathrm{~km} \mathrm{SW}$ of station building, 19.VII.1999, G. P. Schultz y W. Law 1222 (UCR). Municipio Othón P. Blanco, Laguna Guerrero, 20 km al NE de Chetumal, 28.VIII.1979, H. Quero et al. 2777 (MEXU). Municipio Puerto Morelos, Puerto Morelos, 12. III.1900, E. A. Goldman 627 (F); Puerto Morelos, 5.III.1899, C. F. Millspaugh 1714 (F). Municipio Solidaridad, Laguna de Macanxok, 4.X.1974, A. Barrera et al. 257 (MEXU); 1 km al $S$ de Akumal, carretera Cancún - Tulum, 12.II.1981, E. Cabrera y G. Ibarra 1148 (MEXU); Cobá, 9.IX.1980, E. Ucán 355 (MEXU). Municipio Tulum, Tulum, VII.1974, W. L. Boege 3255 (MEXU); Tulum, entre el pueblo y el mar, $1.5 \mathrm{~km}$ al $\mathrm{S}$ de las ruinas, 21.VIII.1981, C. Chan 822 y V. Rico (MEXU); sea cliffs behind the beach by the ruins at Tulum, 17.II.1997, R. M. King y R. M. Garvey 10659 (MO); $3 \mathrm{~km} \mathrm{SSW}$ of the Tulum ruins along a rocky area of the Caribbean Sea, 7.I.1973, R. J. Taylor y C. S. E. Taylor 12601 (MO). San Luis Potosí, municipio Río Verde, $30 \mathrm{~km}$ al NNE de Río Verde, 6.VIII.1960, F. Takaki 292 (MEXU). Sinaloa, municipio Culiacán, dike running parallel to road C19, road to Costa Rica and EL Dorado, $3 \mathrm{mi} \mathrm{W}$ of junction of highway 15 and road
C19 at Las Cascabeles, 23.VIII.1981, D. J. Keil y M. Luckow 15054 (MEXU); km 22 carretera Culiacán - El Dorado, 18. VII.1984, R. Vega 1226 (MEXU). Sonora, municipio Puerto Peñasco, Gran Desierto NE side of La Salina a large inland saltflat-playa with scattered freshwater pozos, 12.XII.1986, R. S. Felger 554 (MEXU). Municipio San Luis Río Colorado, Laguna Prieta, Gran Desierto, NW de Sonora, E. Ezcurra 280 (MEXU, MO); Laguna Prieta, ca. 20 km SE of San Luis Río Colorado, 14.V.1982, R. S. Felger 754 (MEXU); 5 km S of El Doctor, $35 \mathrm{~km}$ S of Rillito or $80 \mathrm{~km}$ S of San Luis Rio Colorado, 10.V.1985, R. S. Felger y K. Van Houten 1048 (MEXU); Sonoran Desert, Gran Desierto, Ciénega El Doctor, west of hwy 3, ca. $61 \mathrm{~km} \mathrm{SSE}\left(172^{\circ}\right)$ of San Luis Río Colorado, 14. III.2010, B. T. Wilder et al. 25 (ARIZ). Tabasco, municipio Cárdenas, km $6.7 \mathrm{~km}$ de la carretera Cárdenas - Coatzacoalcos, 27.VI.1980, C. P. Cowan 3104 (MEXU, MO, NY). Municipio Centla, Arroyo San Pedrito, 4.VI.1998, M. A. Guadarrama et al. 6445 (MEXU); $4.5 \mathrm{~km}$ de la Estación de la Reserva de la Biosfera Pantanos de Centla, atravesando el dren, 1.VII.1998, M. A. Guadarrama y N. Muñiz 6562 (MEXU); Arroyo Polo, a la derecha del puente El Coco, 29.VII.1998, M. A. Guadarrama et al. 6574 (MEXU); orilla de la comunidad El Faisán, 30.VII.1998, M. A. Guadarrama et al. 6626 (MEXU); Laguna de San Pedrito, al $S$ de la estación biológica Pantanos de Centla, 12.III.1998, A. Novelo y L. Ramos 2153 (MEXU, MO); Lagunas Pajaral I y II, aproximadamente 20 $\mathrm{km}$ al $\mathrm{S}$ de Frontera, laguna que se desemboca al Río San Pedrito, 8.VII.1998, A. Novelo y L. Ramos 2703 (MEXU, MO); El Librillo, aproximadamente a $1 / 2$ hora en lancha de la estación Tres Brazos, 21.IX.1998, A. Novelo y L. Ramos 2968 (MEXU, MO); Laguna del Morral, cerca del Arroyo Pantoja, aproximadamente a 1/2 hora en lancha de la estación Tres Brazos, 21.IX.1998, A. Novelo y L. Ramos 2997 (MEXU, MO); Laguna Paquial, cerca de Quintín Arau, aproximadamente a 1 hora en lancha de la estación Tres Brazos, 22.IX.1998, A. Novelo y L. Ramos 3042 (MEXU, MO); Laguna El Guau, aproximadamente a 1.5 horas por lancha al $S$ de la estación Tres Brazos, 23.IX.1998, A. Novelo y L. Ramos 3060 (MEXU, $\mathrm{MO})$; laguna sin nombre, cerca de la laguna Puerta Escondida, aproximadamente $4 \mathrm{~km}$ al $\mathrm{S}$ de la estación Tres Brazos, 25.IX.1998, A. Novelo y L. Ramos 3145 (MEXU, MO); laguna sin nombre al fondo del canal de San Pedrito, el cual desemboca al Río San Pedro, aproximadamente $6 \mathrm{~km}$ al SE de 
la Estación Biológica Tres Brazos, 29.X.1998, A. Novelo y L. Ramos 3181 (MEXU, MO); Arroyo Palmahuapo, aproximadamente 45 min por lancha sobre el Río Usumacinta al $\mathrm{S}$ de la Estación Tres Brazos, 2.XI.1998, A. Novelo y L. Ramos 3372 (MEXU, MO); Laguna La Concepción, aproximadamente $1.5 \mathrm{~h}$ por lancha al $\mathrm{S}$ de la Estación Tres Brazos, 11.XII.1998, A. Novelo y L. Ramos 3507 (MEXU); Laguna Pez Espada, aproximadamente 30 min por lancha al S de la Estación Tres Brazos, 14.XII.1998, A. Novelo y L. Ramos 3589 (MEXU, MO); Reserva de la Biosfera Pantanos de Centla, canal de la Laguna San Pedrito que comunica con Quintín Arau, $8.5 \mathrm{~km}$ al $\mathrm{S}$ de la Estación Central Tres Brazos, 20. IX.2001, A. Novelo et al. 4154 (MEXU). Municipio Centro, La Ceiba, 7.II.1890, J. N. Rovirosa 722 (MEXU). Municipio Frontera, Frontera, 1.V.1982, S. Zamudio 268 (IEB, MEXU). Municipio Jalpa de Méndez, $5 \mathrm{~km}$ al $\mathrm{N}$ de Mecoacam, 6.VI.1981, S. Zamudio 113 (MEXU). Municipio Jonuta, laguna San Sebastián frente al ejido San José aproximadamente $40 \mathrm{~km}$ al SE de Frontera rumbo a Jonuta, 16.V.1998, A. Novelo y L. Ramos 2582 (MEXU, MO). Municipio Macuspana, Laguna Loncho, aproximadamente 1 hora por lancha del sitio llamado el Borde Hormiguero, al N de Ciudad PEMEX, 15. III.1998, A. Novelo y L. Ramos 2241 (MEXU, MO); Laguna Comején, aproximadamente $1 / 2$ por la lancha del Bordo Hormiguero y $30 \mathrm{~km}$ al NE de Ciudad PEMEX, 18.IX.1998, $A$. Novelo y L. Ramos 2873 (MEXU, MO). Municipio Nacajuca, camellones chontales de Tucta, 24.IX.1989, H. Cáliz y $R$. Castillo 255 (IEB); Campo Cen de PEMEX, orilla del Río González, 15.VI.1997, A. M. Hanan et al. 1012 (MEXU); Tecolutla 1a Sección, 27.V.1982, G. Ortiz 551 (MEXU). Municipio Paraíso, Ranchería Moctezuma, 2.5 km dirección S de Paraíso, 16.VII.1979, R. González y R. Miranda 4 (MEXU, MO); Cerro de Tiodomiro, 19.VII.1983, F. Ventura 20471 (ENCB, IEB, MEXU, MO). Tamaulipas, municipio Aldama, Sierra de Tamaulipas: Region of Rancho Las Yucas, 40 km NNW of Aldama, La Minita, E of Las Yucas, 31.VII.1957, R. L. Dressler 2106 (MEXU, MO). Municipio Matamoros, Matamoros, 8.VIII.1888, C. G. Pringle s.n. (F, MEXU). Municipio Nuevo Laredo, $29 \mathrm{~km}$ al O de Nuevo Laredo, 16. IX.1982, F. González 13023 (MEXU). Municipio Tampico, vicinity of Tampico, 1.I.1910, E. Palmer 138 (F, MO); Laguna de Champayán, 3. VI.1989, A. Mora 794 (MEXU). Veracruz, municipio Actopan, Actopan, 6.XI.1970, F. Ventura 2771 (ENCB, MEXU);
Chapopote, 13.VI.1973, F. Ventura 8366 (ENCB, IEB, MEXU). Estación de Investigación sobre Recursos Bióticos Morro de la Mancha, 14.IX.1986, J. L. Villaseñor 965 (MEXU). Municipio Agua Dulce, Río Tonalá, por Arroyo Blasillo, VII.1983, M. A. Cházaro 3297 (MEXU). Municipio Alvarado, 23 km de Alvarado, rumbo a Veracruz-La Piedra-Apompal, 6.VIII.1971, A. Lot 1374 (MEXU). Municipio Coatzacoalcos, Higueras, 1 km al S de Fertimex, 6.V.1984, C. Gutiérrez 1448 (MEXU); along the Trans - Isthmian highway, $1 \mathrm{~km}$ SW of Coatzacoalcos, 5.VIII.1958, R. M. King 1139 (MEXU); Laguneta en la congregación del Mundo Nuevo, 15.VI.2003, C. H. Ramos y E. Martínez S. 2630 (MEXU, MO); alrededores de La Bocatoma, 29.XII.1998, M. Sinaca et al. 2081 (MEXU); La Cangrejera, alrededor de las Torres de alta tensión, 2.IV.1991, M. Sinaca et al. 2197 (MEXU). Municipio La Antigua, Antigua, Gulf Coast, IX.1912, C. A. Purpus 6034 (F, MO); carretera de terracería San Vicente - El Hatillo, 1 km de San Vicente, 20.V.1987, P. Zamora 463 (IEB, MEXU). Municipio Pajapan, Laguna del Ostión, 28.III.1980, L. Gutiérrez y J. Dorantes 59 (MEXU, MO). Municipio Pánuco, 6 km de Tamos, cerca de la desviación a Tuxpan, 12.VI.1986, C. Gutiérrez y E. Montoya 1882 (IEB); near Ebano, 28.II.1939, H. LeSeur 512 (F). Municipio Paso de Ovejas, Paso Real, camino al rancho El Tulipán, 19.III.1987, S. Vargas 520 (IEB). Municipio Tlacotalpan, $1 \mathrm{~km}$ del poblado de San Antonio en el margen meridional de la laguna La Cerca, 20.XI.2004, L. Cruz et al. 19 (MEXU). Municipio Veracruz, Veracruz, 28.III.1910, C. R. Orcutt 3160 (MEXU). Municipio Xalapa, $3 \mathrm{~km}$ al SE de la Colonia 6 de enero, 12.XII.1989, L. Orea et al. 250 (MEXU). Yucatán, municipio Abalá, $15 \mathrm{~km}$ al $\mathrm{S}$ del poblado de Yaxcopoil, a lo largo de la carretera Mérida - Muna, 7.XI.2001, G. Carnevali et al. 6454 (MEXU). Municipio Axcanu, Chunchucmil, rumbo a San Simón, 5.VIII.1986, C. Chan 6992 (MEXU). Municipio Celestún, 15 km al E de Celestún, 16.V.1997, E. Reyes 674 (MEXU). Municipio Dzemul, N of Dzemul, E of Progreso, 28.X.1984, A. H. Gentry y E. M. Zardini 48919 (MO). Municipio Dzidzantun, $500 \mathrm{~m}$ al S de Santa Clara, camino a Chabihao, 24.VII.1987, E. Cabrera y H. de Cabrera 13744 (MEXU, MO). Municipio Mérida, Xlacah, 2.IX.1956, O. Enríquez 755 (MEXU). Municipio Progreso, Chuburná Puerto, 27.I.2011, L. M. Calvo 150 (MO); flats S of Cienega, VII.1938, C. L. Lundell y A. Lundell 8025 (F). Municipio San Felipe, 2 km al E del entronque de San Felipe, rumbo a Río Lagartos, 9.II.1992, 
R. Durán et al. 1519 (MEXU). Municipio Telchac Puerto, 10 $\mathrm{km}$ al O de Telchac Puerto, 13.III.1997, E. Reyes 651 (MEXU). Municipio Tizimin, km 23 de la carretera El Cuyo - Colonia Yucatán, 10.VII.1992, P. Simá et al. 1403 (MEXU).

Pluchea parvifolia (A. Gray) Goodfrey, J. Elisha Mitchell Sci. Soc. 68(2): 252. 1952.

इ Pluchea subdecurrens var. parvifolia A. Gray, Proc. Amer. Acad. Arts 5: 160. 1862. TIPO: MÉXICO. Baja California Sur, Cape St. Lucas and Lower California, VIII.1859, L. J. Xantus 53 (holotipo: GH-00011329!, isotipos: NY-00232539!, US00129413!).

इ Pluchea adnata var. parvifolia (A. Gray) S.F. Blake, Contr. U.S. Natl. Herb. 23(5): 1510. 1926.

इ Pluchea salicifolia var. parvifolia (A. Gray) S.F. Blake, J. Wash, Acad. Sci. 21: 328. 1931.

Arbustos, 1-1.5 m de alto; tallos alados; ramas tomentulosas; hojas sésiles, láminas linear-lanceoladas, 2.7-11.5 × 0.3-2.4 cm, ápice agudo, base cuneada, decurrente, margen serrado, pilosas en ambas superficies; inflorescencias corimbiformes con 9-36 cabezuelas, pedúnculos individuales 0.5$2.6 \mathrm{~cm}$ de largo, pilosos; involucro campanulado 6.4-7.5 $\times$ 8.4-13.6 mm; filarios 60-62, 6-7 seriados, imbricados, lanceolados, ápice agudo, pilosos y glandulares; flores periféricas 100-120, corolas 3.5-4 mm de largo; cipselas 0.36-0.5 mm de largo, pardas, pilosas; vilano con 8-10 cerdas capilares, 3.2$4.5 \mathrm{~mm}$ de largo; flores del disco 18-20, corolas 4.6-5.5 mm de largo, rosadas, lóbulos ovados, glandulares en el ápice; anteras (1.7-)2.1-3 mm de largo; estilo 5.1-6.5 mm de largo; cipselas $0.5-0.63 \mathrm{~mm}$ de largo, pardas o negras, pilosas; vilano con 8-10 cerdas capilares, (3.6-)4-5.5 mm de largo.

Comentarios taxonómicos: Pluchea parvifolia fue descrita como una variedad de $P$. subdecurrens Cass., considerando únicamente características de las hojas (Gray, 1862). Años después, cuando Blake (1930) sugirió sustituir $P$. subdecurrens con $P$. salicifolia, propuso la combinación P. salicifolia var. parvifolia (Blake, 1931). Sin embargo, a mediados del siglo XX Goodfrey (1952) la elevó a rango de especie, considerando que la forma y longitud de las hojas era constante en el tallo y la inflorescencia; además, el in- dumento de los pedúnculos individuales es similar al del involucro, al menos en las series externas. Por otra parte, P. parvifolia y $P$. salicifolia, también difieren en número de flores periféricas (100-120 vs. 225-242); cantidad de flores del disco (18-20 vs. 12-16), cerdas en el vilano (8-10 vs. 1012) y distribución geográfica (Figs. 3, 8).

Distribución: Pluchea parvifolia se encuentra únicamente en Baja California Sur, en elevaciones de 225-2300 m s.n.m. (Fig. 3).

Hábitat: bosques de pino-encino, encinares, matorrales xerófilos, selvas bajas caducifolias y vegetación hidrófila. En suelos arenosos y derivados de granito, cerca de corrientes de agua u orilla de arroyos.

Fenología: florece y fructifica de marzo a agosto.

Ejemplares examinados: MÉXICO. Baja California Sur, municipio La Paz, El Saltito, Rancho La Burrera, 30.V.2008, M. Domínguez 4093 (HClB); Poza La Saya, Cañón de La Burrera, cerca de Todos Santos, 13.V.1986, R. Domínguez 222 (HCIB, MEXU); Poza La Saya, Cañón de La Burrera, 12.V.1993, R. Domínguez 521 (HCIB); La Laguna, trail from La Barrera, 29.VI.1972, A. J. Gilmartin 1859 (MEXU); Sierra La Laguna, Cañón de La Burrera, NE de Todos Santos, 31.III.1987, J. L. León 2484 ( $\mathrm{HClB}, \mathrm{MEXU}) ;$ Paraje de Jacinto, Cañón de La Burrera, 31.III.1987, J. L. León 2501 (MEXU); Agua del Palmillar, subida a La Sierra, 28.IV.1989, J. L. León 3813 (HCIB). Municipio Loreto, Aguaje de Los Encinos, vicinity of Rancho de Los Encinos, Valle de Los Encinos, $\mathrm{S}$ side of Cerro Giganta, 6.VI.1963, A. Carter y R. S. Ferris 3442 (MEXU); slopes of Arroyo de las Palmas, $W$ side of Cerro de la Giganta, Sierra Giganta, 22.IV.1955, A. Carter y J. Reese 4556 (MEXU). Municipio Los Cabos, Cape Region, about $6 \mathrm{mi}$ SW wardly from Santiago, Arroyo San Mateo from Agua Caliente, s.f., Anónimo 7703 (NY); Arroyo Santa Rita, San Jorge W of Santiago, 10.IV.1955, A. Carter y R. S. Ferris 3308 (MEXU); Cañón de San Dionisio, Rancho El Refugio "Arroyo", 1.VII.2008, M. Domínguez 4141 ( $\mathrm{HClB}$ ); Cañón de La Zorra, Arroyo al E de la subida a San Juanito, 20.IV.1989, R. Domínguez 947 ( $\mathrm{HClB})$; vicinity of reservoir, Las Cuevas, Cape District, 19.III.1939, H. S. Gentry 4343 (MEXU); San Bartolo, Cape 


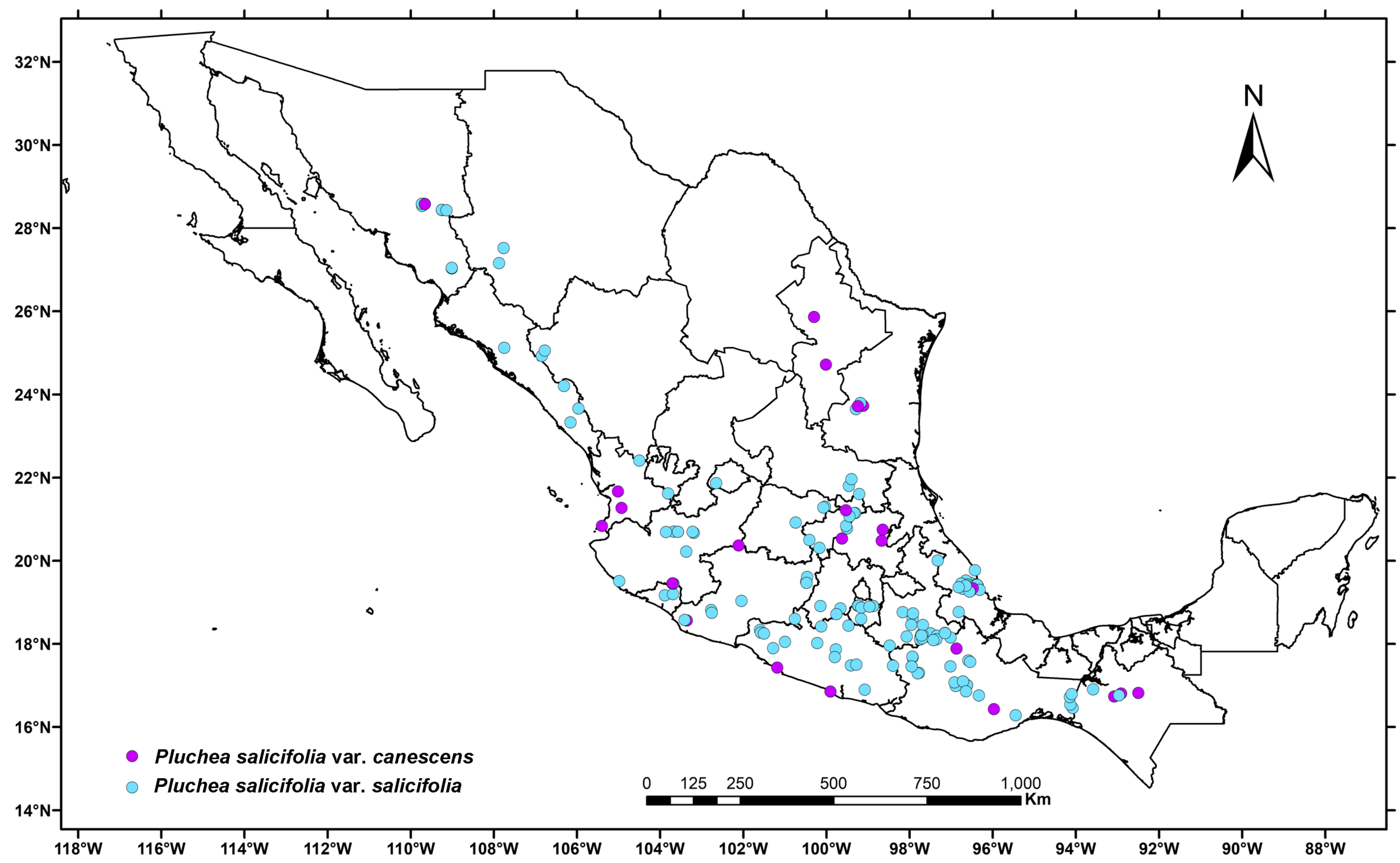

Figura 8: Mapa de distribución geográfica en México de Pluchea salicifolia (Mill.) S.F. Blake var. salicifolia y Pluchea salicifolia (Mill.) S.F. Blake var. canescens (A. Gray) S.F. Blake. 
District, 6.V.1952, H. S. Gentry 11864 (NY); Cape Region, El Reparito, W from Caduano on the E slopes of the Sierra de La Victoria, 8.V.1959, J. Thomas 7814 (MEXU); between Cabeza Ballena and Punta Palmilla, 18.III.1974, G. L. Webster 19529 (MEXU).

Pluchea salicifolia (Mill.) S.F. Blake, Contr. U.S. Natl. Herb. 26(5): 237. 1930.

三 Conyza salicifolia Mill., Gard. Dict. (ed. 8). No. 6. 1768. TIPO: MÉXICO. Veracruz, grows naturally at La Vera Cruz in New Spain, 1731, W. Houston s.n. (holotipo: BH; foto E-0531438!).

= Baccharis adnata Humb., Bonpl.ex Will., Enum. PI. 2: 870. 1809. TIPO: MÉXICO. Morelos, Istla, s.f., F. W. H. A. Humboldt y A. J. A. Bonpland 3972 (holotipo: B-W15557020!, isotipos: P-00322296!, P-00659914!, P-00659915!, P-00659916!, P-00659917!).

三 Conyza adnata (Humb., Bonpl. ex Will.) Kunth, Nov. Gen. Sp. (folio ed.) 4: 58. 1820.

= Pluchea subdecurrens Cass. Dict. Sci. Nat. (ed. 2) 42: 4. 1826. TIPO: No localizado.

इ Pluchea adnata (Humb., Bonpl. ex Will.) C. Mohr, Contr. U.S. Natl. Herb. 6: 790. 1901.

Arbustos, 0.3-2.5(-4) m de alto; tallos alados; ramas pilosas, tomentulosas o tomentosas; hojas sésiles, láminas lanceoladas o linear-lanceoladas, 4.2-22.4 × 0.6-2.1(-5.2) $\mathrm{cm}$, ápice agudo, base cuneada, decurrente, margen serrado, pilosas en ambas superficies o en ocasiones tomentosas en el envés; inflorescencias corimbiformes con 20-300 cabezuelas, pedúnculos individuales $0.25-1.5 \mathrm{~cm}$ de largo, pilosos y glandulares o tomentosos; involucro campanulado 4.2-6.5 × 4.1-7.6 mm; filarios (32-)36-60, 3-4 seriados, imbricados, linear-lanceolados, ápice agudo, piloso-glandulares o tomentoso-glandulares; flores periféricas 225-330, corolas 3.4-4 mm de largo; cipselas (0.36-)0.5-0.7 mm de largo, pardas o negras, pilosas; vilano con 10-12 cerdas capilares, 2.8$4 \mathrm{~mm}$ de largo; flores del disco 10-16, corolas 3.6-5 mm de largo, rosadas, lóbulos ovados, glandulares y papilosos en el ápice; anteras 2-2.5 mm de largo; estilo (3.8-)4.2-6 mm de largo; cipselas 0.5-0.8 mm de largo, pardas o negras, pilosas; vilano con 10-12 cerdas capilares, 3.4-4.8 mm de largo.
Comentarios taxonómicos: Pluchea salicifolia es una especie polimórfica; se reconocen dos variedades, las cuales se distinguen principalmente por el tipo de indumento que presentan en ramas, envés de las hojas y filarios. En la variedad típica es piloso y glandular, mientras que, en la otra, es tomentoso y glandular. Además, también difieren en el número de filarios (32-40 vs. 54-60) y flores periféricas (225-242 vs. 300-330).

\section{Clave para identificar variedades de Pluchea} salicifolia

1a. Ramas pilosas; envés de las hojas y filarios pilosos y glandulares; filarios $\leq 40$

$$
\text { P. salicifolia (Mill.) S.F. Blake var. salicifolia }
$$

1b. Ramas tomentosas; envés de las hojas y filarios tomentosos y glandulares; filarios $\geq 54$....... P. salicifolia (Mill.) S.F. Blake var. canescens (A. Gray) S.F. Blake

\section{Pluchea salicifolia (Mill.) S.F. Blake var. salicifolia}

Arbustos, 0.3-2.5(-4) $\mathrm{m}$ de alto; ramas pilosas; láminas $4.2-22.4 \times 0.6-2.1(-5.2) \mathrm{cm}$, pilosas en el haz, pilosas y glandulares en el envés $\mathrm{o}$ en ocasiones tomentulosas y glandulares; inflorescencias con 20-300 cabezuelas, pedúnculos individuales pilosos y glandulares; involucro 4.2-5.8 $\times$ 4.1-7.6 mm; filarios 32-40, pilosos y glandulares; flores periféricas 225-242; flores del disco 12-16, corolas 3.6-5 mm de largo, lóbulos glandulares en el ápice.

Distribución: en México, Pluchea salicifolia var. salicifolia se encuentra en Aguascalientes, Chiapas, Chihuahua, Colima, Durango, Guanajuato, Guerrero, Hidalgo, Jalisco, México, Michoacán, Morelos, Oaxaca, Puebla, Querétaro, San Luis Potosí, Sinaloa, Sonora, Tamaulipas y Veracruz, en elevaciones de 5-3100 m s.n.m. (Fig. 8).

Hábitat: bosques mesófilos de montaña, de pinoencino, encinares, selvas bajas caducifolias, matorrales subtropicales y xerófilos, vegetación hidrófila, secundaria y ecotonos. Creciendo a orilla de cuerpos de agua o lugares húmedos, en suelos arenosos, arcillosos, calizos o pedregosos, someros o con abundante materia orgánica. 
Fenología: florece y fructifica de noviembre a mayo.

Ejemplares examinados: MÉXICO. Aguascalientes, municipio Calvillo, Arroyo Malpaso, 13.II.2008, J. Martínez 1360 (IEB). Chiapas, municipio Chiapa de Corzo, Chorreadero de Tuxtla, $5.6 \mathrm{mi}$ E of Chiapa de Corzo, along Mexican highway 190, 20.II.1965, D. E. Breedlove 9101 (F); Chorreadero de Tuxtla, $5.6 \mathrm{mi}$ E of Chiapa de Corzo, along Mexican highway 190, 20.II.1965, P. H. Raven y D. E. Breedlove 20134 (F). Municipio Cintalapa, along highway 190, $2 \mathrm{mi} E$ of the Oaxaca border, 24.I.1979, T. B. Croat 46288 (MEXU); along dirt road from Rizo de Oro to Colonia Rodulfo Figeroa (ca. $11 \mathrm{mi} \mathrm{N}$ of Rizo de Oro), 5.5 to $9 \mathrm{mi} \mathrm{N}$ of Rizo de Oro, 15. II.1979, T. B. Croat 47578 (MO). Municipio Ocozocoautla de Espinosa, campamento Bajada del Macho al SE por la ribera del Río Cintalapa a Cascada Laguna Azul, Reserva del Ocote, 7.V.1983, J. I. Calzada 10058 (MEXU). Chihuahua, Municipio Bocoyna, Barranca del Cobre, 6.V.1940, I. W. Knobloch 7094 (MSC). Municipio Guadalupe y Calvo, Barranca del Cobre, puente Río Verde, 14.IV.1976, L. M. Villarreal 17404 (MEXU). Municipio Urique, La Laja de Rodríguez, $5 \mathrm{~km}$ al S de Urique, 1.V.1999, Lebgue 2088 (MEXU). Colima, municipio Colima, Ruta 110 Colima - Pihuamo, km 215 rumbo a Río Salado, 18.I.1982, D. H. Lorence 3799 (F, MO, MEXU). Municipio Comala, Comala, paraje al NW de la población de San Antonio, 22.III.2001, A. G. Miranda y C. López 1107 (MEXU); Rancho El Jabalí, $22 \mathrm{~km}$ airline NNW of Colima in the SW foothills of the Volcán de Colima, 24.I.1991, B. M. Rothschild 111 (MEXU, UCR); Rancho El Jabalí, 22 km airline NNW of Colima in the SW foothills of the Volcán de Colima, near hacienda San Antonio on the highway to Comala, 18.III.1991, A. C. Sanders et al. 10684 (MEXU, UCR); Rancho El Jabalí, $22 \mathrm{~km}$ airline NNW of Colima in the SW foothills of the Volcán de Colima, Colima/Jalisco line passes through ranch, 14.VII.1991, L. Vázquez y B. L. Phillips 875 (MEXU, UCR). Municipio Coquimatlán, 4 km antes de Pueblo Juárez, 23.XI.1994, M. Navarrete 734 (MEXU). Municipio Tecomán, Tecolapa, XII.1985, F. Leger 944 (IEB). Durango, municipio EI Mezquital, San Lucas de Jalpa, Rincón del Río Huazamota, 10.III.2011, D. Ramírez 3611 (IEB). Municipio Tamazula, steep side canyons of the Río Tamazula between La Bajada and La Junta, 20.III.1972, D. E. Breedlove 24541 (MEXU,
MO); SE de El Chapotal, por al camino a Ahuatenipa, 10. III.2002, M. S. González 6653 (IEB). Estado de México, municipio Ixtapan de la Sal, Cerro Arenal, Ixtapan de la Sal, 21.XII.1952, E. Matuda 27432 (MEXU, NY). Municipio Tejupilco de Hidalgo, Tejupilco, distrito Temascaltepec, 26. XII.1936, G. B. Hinton 3025 (MEXU, MO). Municipio Zacualpan, Cerro La Corona, 1.II.1954, E. Matuda 30276 (MEXU). Guanajuato, municipio San Miguel de Allende, un escalón arriba del Cañón del Ingeniero, 1.V.1979, J. Kishler 591 (MEXU); El Charco del Ingenio, IV.2005, W. L. Meagher 3104 (IEB); loc. cit., III.2006, W. L. Meagher 3121 (IEB). Municipio Xichú, Santa Rosa, 9.II.1989, E. Ventura y E. López 6582 (IEB, MEXU); Río Bajo, 5.V.1990, E. Ventura y E. López 7937 (IEB, MEXU). Guerrero, municipio Alcozauca de Guerrero, $1 \mathrm{~km}$ sobre el camino San José Laguna - Alcozauca, 24.II.1984, $R$. E. González 480 (MEXU). Municipio Ayutla de los Libres, Tepango, 14.IV.2000, M. A. Álvarez 197 (MEXU). Municipio Coahuayutla de Guerrero, La Vainilla, $1.55 \mathrm{~km}$ al N, 25.I.1999, J. Calónico 13464 (MEXU); Amatepec, 5.91 km al E, 16.II.1999, J. Calónico 13818 (MEXU); Antonsimon, 1.56 km al NO, 17.II.1999, J. Calónico 13894 (MEXU). Municipio Coyuca de Catalán, NO del puente Los Mangos, 23 km al SW de Zetina y a $72 \mathrm{~km}$ al SW de Ciudad Altamirano, carretera Ciudad Altamirano - Zihuatanejo, 18.I.2010, J. C. Soto 17518 (MEXU). Municipio Cutzamala de Pinzón, cerca de Potrerillos, $3.5 \mathrm{~km}$ al NE de San Lucas y $2.5 \mathrm{~km}$ al SW de Cuirindichapio, camino San Lucas Cuirindichapio - Atotonilco, 20. IV.2011, J. C. Soto 18536 (MEXU). Municipio Eduardo Neri, Amatitlán, $0.35 \mathrm{~km}$ al O, 27.I.1995, A. A. Aguilar 17 (MEXU). Municipio Iguala de la Independencia, Cañón de La Mano, entre Los Amates y El Naranjo, $10 \mathrm{~km}$ al $\mathrm{N}$ de Iguala por el ferrocarril, 21.II.1987, C. Catalán et al. 655 (IEB, MEXU, MO). Municipio José Azueta, San Antonio, $9 \mathrm{~km}$ al NE de Vallecito de Zaragoza, 17.IV.1986, J. C. Soto et al. 12460 (IEB, MEXU). Municipio Leonardo Bravo, Los Morros, 53 km de la desviación a Filo de Caballos de la carretera Mezcala - Chilpancingo, 29.III.1981, S. Torres 518 (MEXU). Municipio Mochitlán, Tepechicotlán, 28.II.1970, E. Halbinger 326 (IEB, MEXU, MO). Municipio San Miguel Totolapan, $4.5 \mathrm{~km}$ al SE de Santa María de Las Flores, El Querengue, 24.II.1984, M. G. Campos 1146 (MEXU). Municipio Tixtla de Guerrero, La Estacada, Región Centro, 24.I.1997, I. Villalba 113 (MEXU). 
Municipio Tlapehuala, $1.7 \mathrm{~km}$ E of Almoloya on route 51 in route to Iguala, 19.I.1992, L. A. Prather y J. A. Soule 1192 (MEXU). Municipio Xochihuehuetlán, $1.5 \mathrm{~km}$ al S de Jilotepec, carretera Palomas - Tlapa, 27.II.1994, E. Moreno y M. A. Monroy 777 (MEXU). Hidalgo, municipio Tecozautla, Tecozautla, 25.XII.1987, E. Argüelles 3054 (F, MEXU). Jalisco, municipio Jocotepec, ribera de La Laguna de Chapala, 2. VI.1996, L. M. Villarreal 17010 (MEXU). Municipio La Huerta, sobre el lindero NE de la Estación de Biología Chamela UNAM, 16.II.1998, J. Calónico 7913 (MEXU). Municipio San Martín de Bolaños, La Joya $1 \mathrm{~km}$ al S por el arroyo principal, $12 \mathrm{~km}$ al SE de San Martín de Bolaños, 25.V.1990, A. Flores y G. Martínez 1785 (MEXU). Municipio Tala, Arroyo de los Chorros de Tala, 9.III.1975, L. M. Villarreal 13669 (MEXU). Municipio Teuchitlán, nacimiento del Río Ameca, baños termales, 4.IV.1976, L. M. Villarreal 8467 (MEXU). Municipio Tonalá, rancho San José del Potrero, Arroyo Tepalcates, bajando del Potrero de Las Moras hacia el Río Santiago, 4. III.2003, P. Carrillo y L. Ortiz 3903 (IEB); Las Tinajas, 6. III.1966, L. M. Villarreal 173 (IEB, MEXU). Municipio Zapopan, spa Río Caliente, $25 \mathrm{~km}$ al N de Guadalajara, 18. IV.1973, W. L. Boege 2809 (MEXU). Michoacán, municipio Aguililla, $4 \mathrm{~km}$ al NO de Aguililla, 9.IV.1985, J. C. Soto et al. 8066 (MEXU, MSC); 1.5 km en línea recta al NO de La Paz, Arroyo El Salto, alrededores de las cascadas, 1.IV.2004, V. W. Steinmann y J. M. Porter 4206 (ARIZ, IEB, MEXU). Municipio Aquila, Río La Joya, 5.XII.1979, B. Guerrero 361 (MEXU). Municipio Jungapeo, canyon below San Jose Purúa, 27.III.1961, R. L. MacGregor 16546 (MEXU). Municipio Los Reyes, cerca del Río Apupátaro, 21.I.2004, I. García y A. Linares 6326 (IEB). Municipio Múgica, ladera al $\mathrm{N}$ del Río El Marqués, $400 \mathrm{~m}$ al SE del puente sobre la autopista Morelia - Lázaro Cárdenas, 1.II.2004, V. W. Steinmann y J. M. Porter 4100 (ARIZ, IEB, MEXU). Municipio Tuxpan, cañada del Puerto de la Cantera, sobre la carretera Ciudad Hidalgo - Zitácuaro, 23.IV.1990, H. Díaz y E. Pérez 6157 (IEB, MEXU). Municipio Zitácuaro, San José Purúa, 10.III.1984, J. C. Soto y A. Román 6224 (MEXU). Morelos, municipio Cuernavaca, Valle de Cuernavaca, IV.1929, E. Lyonnet 382 (MEXU); by stream near Cuernavaca, 14.II.1899, C. G. Pringle 8003 (F, MEXU, MSC). Municipio Jojutla, falda del cerro de Jojutla, 12.XII.1968, J. Vázquez 2163 (MEXU). Municipio
Tepoztlán, paraje Xaltepetlaoxtoc, poblado próximo a Santa Catarina, 1.III.1987, M. L. Espín y E. Cedillo 52 (MEXU). Municipio Yautepec, Oaxtepec, s.f., F. Gallegos 11 (MEXU); loc. cit., 17.I.1953, F. Gallegos 640 (MEXU); cerca de Oaxtepec, 16.III.1952, E. Matuda 26008 (MEXU); Yautepec, a $20 \mathrm{~m}$ del río, 9.II.1988, R. M. Quezada 1917 (MEXU). Oaxaca, municipio Asunción Ixtaltepec, camino al paraje Agua Tibia, $1 \mathrm{~km}$ al N de Nizanda, 4.XI.2002, E. A. Pérez y J. Meave 2270 (MEXU). Municipio Asunción Nochixtlán, Camotlán, 20. XI.1895, L. C. Smith 142 (MEXU). Municipio Concepción Buenavista, Río Grande, 24.I.2010, G. Juárez y S. García 176 (MEXU). Municipio Oaxaca, Hacienda Guadalupe, Valle de Oaxaca, 11.III.1937, C. Conzatti 5253 (MEXU); Hacienda Guadalupe, 4.II.1909, C. Conzatti y H. Conzatii 2490 (F, MEXU); mercado de abastos, procedente de Valles Centrales, 25.V.1988, G. J. Martin 328 (MEXU). Municipio Rojas de Cuauhtémoc, mountain $2 \mathrm{~km}$ WSW of Rojas de Cuauhtémoc, 21.VI.1996, R. A. Werling 628 (ASU). Municipio San Dionisio Ocotepec, Agua Santa, 22.I.1988, A. Flores 1270 (IEB, MEXU). Municipio San Felipe Tejalapa, Barranca León, 17.II.2012, M. Cruz 1007 (MEXU). Municipio San Juan Mixtepec, 3 km al S de San Juan Mixtepec, 18.II.1988, A. J. García et al. 3668 (MEXU); Yunii, Cañada Vieja o antigua Cañada, 31.XII.1988, J. Reyes 1355 (MEXU). Municipio San Juan Quiotepec, San Juan Quiotepec, 15.II.1986, J. García 17 (IEB, MEXU). Municipio San Miguel Chimalapa, Río Escondido, Arroyo Baúl, 0.1-1.0 km al O de su unión con el Río Portamonedas y de Benito Juárez, $38 \mathrm{~km}$ en línea recta al $\mathrm{N}$ de San Pedro Tapanatepec, 18.II.1985, S. Maya 1215 (MEXU). Municipio San Pablo Cuatro Venados, canyon of the Río Zavaleta near the village of San Pablo Cuatro Venados, 15-18 km WSW of Oaxaca, 20.I.1927, W. H. Camp 2447 (NY). Municipio Santa María Chimalapa, El Gigante, embocadura de Arroyo Los Pericos al Río Portamonedas, 9 km en línea recta al NE de Benito Juárez, 47 km en línea recta al NNE de San Pedro Tapanatepec, 5.III.1987, S. Maya 4244 (MEXU, MO). Municipio Santiago Chazumba, $3.9 \mathrm{~km}$ al O de la entrada en el km 63.5 carretera Tehuacán - Huajuapan hacia Bustamante, 22.VI.2006, M. Ayala et al. 347 (MEXU); Agua del Coyote, $1.5 \mathrm{~km}$ al O de Olleras de Bustamante, 29.IV.2006, P. Carrillo y D. Cabrera 6047 (MEXU); Cañada del Coyul, agencia de policía El Higo, 7.II.1999, E. Guízar y A. G. Miran- 
da 4405 (IEB, MEXU). Municipio Santiago Comaltepec, Santiago Comaltepec, 23.XI.1987, L. Hernández y J. G. Martin 144 (MEXU). Municipio Santo Domingo Tehuantepec, El Zacatal, Rancho Limón, $17 \mathrm{~km}$ al O de Tehuantepec, 18. II.1986, C. Martínez 418 (IEB, MEXU). Municipio Santo Domingo Tonalá, Puente Morelos presa El Boquerón, $3 \mathrm{~km}$ al NE de Tonalá carretera a Huajuapan, 9.II.1993, J. I. Calzada y A. Campos 18307 (MEXU); Barranca Yubijaza hasta los Limoncillos cuesta Camino Real, 1.II.2009, L. A. Hernández y A. Torres 885 (MEXU). Municipio Santo Domingo Yodohino, Paraje Aquelado, $1 \mathrm{~km}$ al NE de la localidad, poblado próximo Santo Domingo Yodohino, 12.Il.2006, J. López 237 (MEXU). Municipio Santo Tomás Jalieza, Paraje Piedra Blanca, $1 \mathrm{~km}$ al $\mathrm{N}$ del tanque de cría de mojarra, Santo Tomas Jalieza, Valles Centrales, 11.III.2006, C. A. Cruz et al. 2909 (MEXU). Municipio Santos Reyes Tepejillo, 4 km al N de Santos Reyes Tepejillo, senda antigua para San Miguel Tlacotepec y Río Boquerón, 7.II.1997, J. I. Calzada 21678 (MEXU); 3 km de Santos Reyes Tepejillo, senda para las Cuevas del Diablo y Río Boquerón, 17.III.1998, J. I. Calzada 22452 (MEXU). Puebla, municipio Acatlán, Amatitlán, 13.XII.1942, F. Miranda 2505 (MEXU). Municipio Atexcal, Barranca Aguaxotitla, 4 km al O de Nopala, 14.II.1993, P. Tenorio y F. Tenorio 18520 (MEXU). Municipio Caltepec, Santa Lucía, Río Hondo, 11.III.2011, R. Redonda et al. 512 (MEXU); Barranca de los Membrillos, al SO de Caltepec, 5.V.1983, P. Tenorio et al. 3785 (DES, F, MEXU); Barranca de los Membrillos, 12.I.1984, P. Tenorio y C. Romero 5106 (DES, F); Mogote León, Cerro Grande, 10.II.1984, P. Tenorio y C. Romero 5438 (IEB, MEXU). Municipio Coxcatlán, 0.5 mi $S$ of Coxcatlán on highway 131, 11.I.1984, L. Hardison et al. 101 (MEXU); Río de Coxcatlán, 13.I.1983, G. Salazar s.n. (MEXU). Municipio Huehuetlán El Grande, $1 \mathrm{~km}$ al N del Puente de Atotonilco, 9.III.1995, M. S. Hernández 48 (MEXU). Municipio Hueytamalco, campo experimental Las Margaritas, Instituto Nacional de Investigaciones Forestales Agrícolas y Pecuarias INIFAP, 16.IV.2008, M. G. Cornejo et al. 2673 (MEXU). Municipio Santiago Chazumba, $9 \mathrm{~km}$ al NE de Chazumba, $1 \mathrm{~km}$ después del límite estatal Oaxaca-Puebla, 22.III.1980, F. González et al. F-688 (MEXU); 1 km por la desviación a Acaquizapan, de la carretera Huajuapan de León - Tehuacán, $5 \mathrm{~km}$ al S de Huapanapan, 20.II.1986, J. L. Villaseñor et al. 910
(MEXU). Municipio Tepexi de Rodríguez, Río Axamilpa, 6.5 km sobre la carretera que va de Tepexi de Rodríguez a Molcaxac, 18.I.1991, J. Castrejón 8 (MEXU); Agua Escondida, 2 $\mathrm{km}$ al $\mathrm{S}$ de la comunidad Todos Santos Almolonga, 13. XI.2002, C. Mota 162 (MEXU); Cañada Puente de Dios, arriba, 19.XII.1982, R. Medina y A. Valiente 414 (MEXU). Municipio Zapotitlán, Chiltepin, IV.1908, C. A. Purpus 3121 (F, MO, NY). Querétaro, municipio Cadereyta de Montes, Maconi, 26.III.1985, R. Fernández 2857 (ENCB, MEXU, NY); La Solana, 7.V.1991, M. A. Salcedo 126 (IEB); cañón del Río Moctezuma, bajando por la ventana 2, 21.III.2006, S. Zamudio 13356 (IEB). Municipio Corregidora, El Batán, 2. III.1977, E. Argüelles 735 (MEXU); 2 km al N de San Francisco, cañón al pie de la presa El Batán, 20.V.1995, L. Hernández 3507 (MEXU). Municipio Jalpan de Serra, S de Tanchanaquito, Los Rejalgares, s.f., L. López 537 (IEB, MEXU); cañón del Río Estórax entre el Arroyo de los Chilares y Las Adjuntas con el Río Moctezuma, 6.III.1996, S. Zamudio 9677 (MEXU). Municipio Landa de Matamoros, Río Moctezuma, S de Matzacintla, 10.IV.1989, E. Carranza 1613 (IEB, MEXU); Paso del Tereguaje, Río Moctezuma, S de Landa, 7.III.1988, A. Herrera 65 (IEB, MEXU); Tangojo, orilla del Río Moctezuma, 4.III.1988, H. Díaz y S. Zamudio 4615 (IEB). Municipio Peñamiller, Junta de Higueras en la rivera del Río Estórax, cerca de Higueras, 9.II.1994, R. Hernández et al. 10472 (MEXU); alrededores de Peñamiller, 11.IV.1977, S. Zamudio 2019 (IEB). Municipio Pinal de Amoles, $1 \mathrm{~km}$ al $S$ de Escanelilla, carretera a Pinal de Amoles, 18.V.1987, J. Rzedowski 43383 (IEB). San Luis Potosí, municipio Tamasopo, Cañón de Los Limones, Rincón de Ramírez, 21.IV.1984, H. Chemis 122 (MEXU); parte alta del área turística Las Cascadas, riberas del Río Tamasopo, en la cercanía a Tamasopo, 10.V.2000, A. Sánchez et al. s.n. (IEB, MEXU). Sinaloa, municipio Concordia, Rincón de la Ventanilla, El Corral, 20.III.1943, S. S. White 5052 (F, MEXU). Municipio Mocorito, Rancho Viejo, 25.III.1984, E. Jasso y J. Montero 5 (MEXU). Municipio San Ignacio, Ajoya, 31.III.1989, R. Vega et al. 3221 (MEXU). Sonora, municipio Álamos, side canyon W from Arroyo la Aduana, upstream from Aduana pueblo, 12.IV.2005, S. Carnaham y C. Smith 1137 (ARIZ); Sierra de Alamos, hillside above and to the $\mathrm{W}$ of Aduana, $6 \mathrm{~km}$ by air W of Alamos, 16.III.1993, V. W. Steinmann y C. Smith 126 
(MEXU). Municipio Guaymas, Cañón de Nacapules, about 6 km NE of Bahía San Carlos, 10.X.1984, R. S. Felger et al. 144 (MEXU). Municipio San Javier, Arroyo San Javier, 1 km al $\mathrm{S}$ del poblado y $1.5 \mathrm{~km} \mathrm{~N}$ del cerro El Verde, 28.IV.1996, L. Varela 2 (MEXU); San Javier, rancho de los Campa a 1.5 $\mathrm{km}$ al S de la carretera, 16 al E del cerro Los Amoles, 22. IV.1997, L. Varela 100 (MEXU). Municipio Onavas, Arroyo del Pilladito, near Tepoca, 27.III.1997, A. L. Reina y T. R. Van Devender 333 (ASU, MEXU, MO). Municipio Yécora, 3.5 km W of Santa Ana road, $4.8 \mathrm{~km}$ E of San Nicolás road on Mexico 16, 31.III.1997, A. L. Reina y T. R. Van Devender 465 (MEXU); Arroyo La Quema (A. Milpillas), near Tepoca, 24.V.1998, A.L. Reina et al. 593 (ASU, NY, USON). Tamaulipas, municipio Ciudad Victoria, Ciudad Victoria - Jaumave, 1932, H. W. Von Rozinski 337 (F). Municipio Jaumave, Cañón de la Libertad, Arroyo San Felipe 10 km al NW de Ciudad Victoria, 21.IV.1996, A. Mora 5722 (MEXU). Veracruz, municipio Actopan, Mata de Caña, ca. $5 \mathrm{~km} \mathrm{SW}$ of Puente Nacional, 4.IV.1979, M. O. Dillon et al. 1857 (F, MO, NY); camino entre Jareros y Los Ídolos, 27.II.1990, L. Orea 391 (MEXU); El Ranchito, 1.IV.1976, F. Ventura 12601 (ENCB, MEXU); Paso de la Milpa, 23.I.1982, F. Ventura 19327 (ENCB, IEB, MEXU). Municipio Alto Lucero de Gutiérrez Barrios, Palma Sola, 31.I.1973, F. Ventura 7770 (ENCB, IEB, MEXU, MO). Municipio Apazapan, Apazapan, 29.I.1979, F. Ventura 15716 (ENCB, IEB, MEXU). Municipio Emiliano Zapata, Puente Nacional, carretera Xalapa - Veracruz, 10. IV.1972, J. Dorantes 527 (F, MEXU); 1 km S of Corral Falso, on road to Pinaltepec, 23.I.1984, M. Nee y K. Taylor 29006 (F, MO, NY); Plan del Río, 20.I.1971, F. Ventura 3008 (ENCB, F); Plan del Río, 11.II.1976, F. Ventura 12439 (ENCB, IEB, MEXU, MO); Plan del Río, al E, 5.II.1982, R. Villanueva 84 (MEXU). Municipio Jalcomulco, $\mathrm{N}$ del puente del Río Los Pescados, III.1984, G. Castillo 3099 (IEB); 2 km al SW de Cantarranas, 30.I.1985, G. Castillo y M. E. Medina 3589 (IEB, MEXU). Municipio La Antigua, Panoaya, s.f., C. A. Purpus 8996 (F, MO); El Ciruelo, 2.II.1976, F. Ventura 12389 (ENCB, IEB, MEXU). Municipio Omealca, orillas del Río Blanco, Xuchiles, donde cruza la carretera Córdoba Omealca, 19.III.2010, J. L. Villaseñor et al. 1797 (MEXU). Municipio Puente Nacional, Puente Nacional, 10.II.1977, F. Ventura 13801 (ENCB, MEXU, MO); Mata de Caña, 27.
II.1980, F. Ventura 16903 (ENCB, IEB, MEXU, MO). Municipio Tlaltetela $1 \mathrm{~km}$ al E del Río Los Pescados, carretera nueva Xalapa - Huatusco, 1.III.1975, V. Sosa 79 (MEXU). Municipio Úrsulo Galván, camino entre La Gloria y Zapotito, 25.I.1990, L. Orea e I. Pérez 236 (MEXU); 100 m de La Gloria, 28.I.1990, L. Orea 294 (MEXU); EI Limón, carretera Cardel - Palma Sola, 29.I.1985, F. Vázquez 2220 (IEB). Municipio Xalapa de Enríquez, Pinaltepec, 9.I.1975, F. Ventura 10777 (ASU, ENCB, MEXU).

Pluchea salicifolia (Mill.) S.F. Blake var. canescens (A. Gray) S.F. Blake, J. Wash. Acad. Sci. 21(14): 328. 1931.

E Pluchea subdecurrens var. canescens A. Gray, Proc. Amer. Acad. Arts 5: 182. 1862. TIPO: MÉXICO. Veracruz, Wartenberg, near Tantoyuca, prov. Huasteca, XII.1858, L. C. Ehrenberg 343 (holotipo: GH-00011331!, isotipos: K-000222145!, P-01816084!, P-01816085!, P-01816086!, P-01816087!, P-01816088!).

Arbustos, 0.5-2.5 m de alto; ramas tomentosas; láminas 6-12.8 $\times 0.7-1.8 \mathrm{~cm}$, pilosas y glandulares en el haz, tomentosas y glandulares en el envés; inflorescencias con 54-236 cabezuelas, pedúnculos individuales tomentosos; involucro 4.8-6.5 × (4.3-)6-7 mm; filarios 54-60, tomentosos y glandulares; flores periféricas 300-330; flores del disco 10-12, lóbulos glandulares y papilosos en el ápice.

Distribución: en México, Pluchea salicifolia var. canescens se encuentra en Chiapas, Guerrero, Hidalgo, Jalisco, Michoacán, Nayarit, Nuevo León, Oaxaca, Querétaro, Sonora, Tamaulipas y Veracruz, en elevaciones de 140-1900 m s.n.m. (Fig. 8).

Hábitat: bosques mesófilos de montaña, de pino-encino, encinares, matorrales xerófilos, selvas altas perennifolias, bajas caducifolias, vegetación hidrófila, secundaria y ecotonos. En suelos arenosos, arcillosos, limosos, pedregosos o salinos, a orillas de cuerpos de agua o lugares húmedos.

Fenología: florece y fructifica de octubre a abril. 
Ejemplares examinados: MÉXICO. Chiapas, municipio Ixtla, arroyo en carretera Tijera de Pichucalco, 10.II.1986, A. Méndez 8805 (MEXU). Municipio Tenejapa, Río Cruz Pilal, 30.I.1983, A. Méndez 5404 (IEB, MEXU, MO). Municipio Tuxtla Gutiérrez, hacia Nido de Águila, E Tuxtla Gutiérrez, 12.I.1950, F. Miranda 5897 (MEXU). Guerrero, municipio Acapulco de Juárez, Acapulco and vicinity, X.1894, E. Palmer 441a (F, MO, NY). Municipio Petatlán, Loma Bonita, 31.I.1982, N. Diego 2498 (MEXU). Hidalgo, municipio Metztitlán, Barranca de Venados, Río Tulancingo, 18.III.1981, J. Bueno y P. Carter, 11 (MEXU). Municipio Tecozautla, Tecozautla, 1.IV.1979, E. ArgüeIles 1220 (MEXU). Municipio Tianguistengo, $8 \mathrm{~km}$ al $\mathrm{N}$ de Tianguistengo, 26.III.1981, R. Hernández y D. Rodríguez 5675 (MEXU, MO). Jalisco, municipio San Sebastián del Oeste, Las Mesitas, NW of San Sebastián, Sierra Madre, 15.III.1927, Y. Mexia 1878 (F, MO, NY). Municipio Zapotitlán, road between La Becerra and lago La María, $22 \mathrm{~km}$ airline NNW of Colima in the SW foothills of the Volcan de Colima, in a moist cleared area at the motel at La María, 7.I.1991, A. C. Sanders et al. 10340 (MEXU, UCR). Michoacán, municipio La Piedad, $10 \mathrm{~km}$ al O de La Piedad, sobre la carretera a Guadalajara, 22.X.1986, J. Rzedowski 41218 (IEB, MEXU). Nayarit, municipio Compostela, 6 km al O de Compostela sobre el camino a las Cumbres de Huicicila, 3.I.1986, O. Téllez 9439 (IEB, MEXU); Compostela, 55 $\mathrm{km}$ al S de Las Varas, camino a Puerto Vallarta, 7.II.1989, O. Téllez y G. Flores 11873 (IEB, MEXU). Municipio San Blas, $3.5 \mathrm{~km}$ al E de Jumatán camino a la carretera Tepic Mazatlán, 4.IV.1987, O. Téllez 10253 (MEXU). Nuevo León, municipio Galeana, La Poza-Río de San José, 1.VII.1992, J. C. Hinton 22085 (IEB). Municipio Monterrey, by streams, Monterrey, 15.IV.1906, C. G. Pringle 10198 (F, MEXU, MO, NY). Oaxaca, municipio Concepción Pápalo, San Lorenzo Pápalo, 30.VIII.2002, L. Solís et al. 457 (MEXU). Municipio Rojas de Cuauhtémoc, mountain $2 \mathrm{~km}$ WSW of Rojas de Cuauhtémoc, 7.IX.1999, R. A. Werling 524 (ASU). Municipio San Bartolo Yautepec, Tanque de Agua, 7.I.2012, D. López 2138 (MEXU). Querétaro, municipio Pinal de Amoles, Ahuacatlán, 24.XII.1982, R. Hernández 9278 (MEXU). Sonora, municipio San Javier, La Barranca, San Javier, cañón profundo sobre la Carretera Federal 16, 25.V.1996, A. Búrquez et al. 137 (MEXU); 2.7 km E of La Barranca on Mexico
16, 9.III.1996, T. R. Van Devender et al. 11 (ASU, MEXU). Tamaulipas, municipio Ciudad Victoria, Cañón del Novillo, 30 km al O de Ciudad Victoria, 9.V.1985, F. González 14513 (MEXU); vicinity of Victoria, 1.Il.1907, E. Palmer 12 (F); Veracruz, municipio Puente Nacional, Puente Nacional, 18.V.1981, S. Hernández y A. Figueroa 40 (F).

Pluchea sericea (Nutt.) Coville, Contr. U.S. Natl. Herb. 4: 128. 1893.

इPolypappus sericeus Nutt., Proc. Acad. Nat. Sci. Philadelphia 4(1): 22. 1848. TIPO: ESTADOS UNIDOS DE AMÉRICA. California: in Upper California, toward Rocky Mountains, s.f., W. Gambel s.n. (holotipo: no localizado).

三 Berthelotia sericea (Nutt.) Rydb., Bull. Torrey Bot. Club 33(3): 154. 1906.

इEremohylema sericea (Nutt.) A. Nelson, Univ. Wyoming Publ. Sci., Bot. 1(3): 54. 1924.

$\equiv$ Tessaria sericea (Nutt.) Shinners, Sida 3(2): 122. 1967.

Arbustos, 1-2.5(-4) m de alto; tallos sin alas; ramas seríceas, ocasionalmente tomentosas; hojas sésiles, rara vez con peciolos 2-6 $\mathrm{mm}$ de largo, láminas lanceoladas o linear-lanceoladas, 2-5.5 cm de largo, $1.6-8 \mathrm{~mm}$ de ancho, ápice agudo, base cuneada, margen entero, pilosas o tomentulosas en el haz; pilosas, tomentosas o seríceas en el envés; inflorescencias corimbiformes con 3-10 cabezuelas, pedúnculos individuales $2.8-5 \mathrm{~mm}$ de largo, seríceos o tomentosos; involucro campanulado $5.2-7.8 \mathrm{~mm}$ de largo, 5.4-8 mm de ancho; filarios 24-26(-38), 3-4 seriados, imbricados, elípticos, ápice agudo, seríceos o tomentosos; flores periféricas 115-120, corolas 3.6-5.2 mm de largo; cipselas 0.6-0.8 mm de largo, pardas, glabras; vilano con 28-30 cerdas capilares, 4-4.4(-5.6) mm de largo; flores del disco 1820, corolas 4.2-5.8(-6.3) mm de largo, rosadas, lóbulos lanceolados, papilosos en el ápice; anteras 1.8-2.5(-3.1) mm de largo; estilo 5.4-7.8 mm de largo; cipselas (0.6-)1-1.6 $\mathrm{mm}$ de largo, pardas, glabras; vilano con (20-)24-30 cerdas capilares, (4-)5-6.2(-7.6) mm de largo (Fig. 1D, F).

Distribución: en México, Pluchea sericea se encuentra en Baja California y Sonora, en elevaciones de 0-1280 m s.n.m. (Fig. 3). 
Hábitat: encinares, matorrales xerófilos, palmares, selvas bajas caducifolias, vegetación de dunas costeras, halófila e hidrófila; en suelos arcillosos, arenosos, aluviales, rocosos, salinos o derivados de granito.

Fenología: florece y fructifica durante todo el año.

Ejemplares examinados: MÉXICO. Baja California, municipio Ensenada, from Ensenada to Guadalupe, 24.V.1936, L. H. Bailey 577 (F); Gulf coast, 46 mi S of Bahía de Los Angeles, 1.IV.1991, S. Boyd y T. Ross 5668 (MEXU); San Andrés canyon above Santa Rosalía, 20.III.1984, D. E. Breedlove 60812 (MEXU, NY); lower Guadalupe Canyon, near hot springs, Sierra de Juárez, E of Laguna Salada, Cantu Palms W of Rancho Poderosa, 13.III.1988, D. Charlton y R. Thorne 1375 (MEXU); $8 \mathrm{mi} \mathrm{N}$ of Rosarito, on Mexican highway 1, 2.V.1992, J. S. Miller et al. 7347 (MEXU); Arroyo San Pedro, near San Pedro, 11.III.1966, R. C. Moran 12524 (MEXU); inner beach dunes San Ramón, 11.X.1977, R. C. Moran 25078 (MSC); Ensenada, 31.VIII.1889, C. R. Orcutt s.n. (MEXU); rte. 1, ca. $19.3 \mathrm{mi}$ S of San Vicente and 2.1 mi N of Colonet, 6.VI.1972, D. J. Pinkava et al. 9018 (ASU, DES); Canyon San Isidro, ca. $3.3 \mathrm{~km}$ NE of Eréndira, in the bottom of the canyon just $\mathrm{S}$ off the main road into Eréndira, 21.III.2012, J. P. Rebman 22842 (UCR); SE of Maneadero, $\mathrm{S}$ of Punta Banda along the coastal dirt road, large canyon with a flowing freshwater stream near El Retiro (Maximinos), 1.II.1994, J. P. Rebman y S. Hirales 2133 (ASU, BCMEX); Catavina, stream crossing on hwy 1, 2.3 miles $N$ of Hotel La Pinta at Catavina, 29.III.1985, A. C. Sanders et al. 2474 (UCR); San Rafael, $80 \mathrm{~km}$ al SE de Bahía de Los Ángeles, 21.IV.1987, P. Tenorio y C. Romero 13064 (BCMEX, MEXU); Sierra Juárez, Palmas de Cantú; E base of Sierra de Juárez ca. 15 mi S of jct of Baja California hwy 2 and Guadalupe Canyon road, 22.III.1986, R. F. Thorne 61640 (BCMEX); Cañón de Guadalupe, dry slopes along canyon above resort area, 23.III.1986, R. F. Thorne 61759 (MEXU); Las Chichihuas, 10.X.1981, F. Uribe s.n. (BCMEX); inner end of Escondido Canyon, $11.1 \mathrm{mi}$ S of Punta Prieta, 23.II.1935, I. L. Wiggins 7718 (F); along stream near Rancho San Jacinto, $45 \mathrm{mi}$ S of Ensenada, 7.IX.1930, I. L. Wiggins y D. Demaree 4757 (COLO, F, NY). Municipio Mexicali, Algodones dunes, southern toe at edge of agricultural field, end of dirt road
$\mathrm{N}$ of Rancho Grande and $\mathrm{N}$ of the road between Merida and Algodones, $4 \mathrm{mi}$ NE of Ciudad Morelos, 10.V.2005, D. S. Cooper s.n. (UCR); El Mayor, Río Hardy, 14.IV.1977, R. S. Felger y A. Rea 5 (MEXU); lower Río Colorado valley, adjacent to large irrigation canal, 13.VII.2006, R. S. Felger 34 (BCMEX); Valle de Mexicali, V.1979, A. Romo 15 (MEXU); near S of Colorado River, 30 km S of Mexicali, 15.IV.1967, S. N. Stephenson 65 (MSC); entre Tecolotes y Mexicali, XII.1975, R. Vargas s.n. (MEXU). Municipio Tecate, La Rumorosa, 16.VI.2014, R. Santos s.n. (BCMEX); Valle de las Palmas, 16.V.1987, P. Tenorio y C. Romero 13449 (MEXU); $18 \mathrm{mi} \mathrm{S}$ of Tecate, near junction of highway 3 and road to Cerro Bola, $2 \mathrm{~km}$ S of Valle de las Palmas, 14.III.1987, R. F. Thorne et al. 62113 (MEXU); $0.5 \mathrm{mi} \mathrm{S}$ of highway near $\mathrm{N}$ end of El Salado dry lake bed, 16.III.1960, I. L. Wiggins y D. B. Wiggins 15735 (MEXU). Municipio Tijuana, margen del Río de Las Palmas, 30.VIII.2003, S. Becerril y R. Uribe 1 (MEXU); dry Tijuana river banks, 28.VII.1912, H. H. Smith 5192 (F). Sonora, municipio Puerto Peñasco, Laguna Prieta, 20 km SE of San Luis Río Colorado, 14.V.1985, R. S. Felger y M. A. Dimmitt 753 (MEXU); above Adair Bay, 11 mi NW of station Gustavo Sotelo, along ferrocarril NW of Puerto Peñasco, 24.III.1980, G. L. Webster 24242 (MEXU). Municipio San Luis Río Colorado, Pozos de La Borrascosa, $50 \mathrm{~km}$ SE de Santa Clara Sonora, Solonchak en los alrededores del manantial, 28.V.1982, E. Ezcurra s.n. (MEXU); $0.5 \mathrm{~km} \mathrm{E}$ of El Golfo de Santa Clara, 13.III.1975, R. S. Felger et al. 79 (ARIZ); Gran Desierto, NE side of La Salina, 12.XII.1986, $R$. S. Felger y T. Bowen 553 (MEXU, MSC); $5 \mathrm{~km} \mathrm{~S}$ of Rillito, or $50 \mathrm{~km} \mathrm{~S}$ of San Luis Río Colorado on road to El Golfo, 6.X.1985, R. S. Felger y K. Van Houten 1045 (MEXU); edge of Río Colorado in San Luis Río Colorado, 27.IV.1997, A. L. Reina y T. R. Van Devender 534 (MEXU, USON); Sonoran Desert, Gran Desierto, Las Salinas, near km 85 on the road from Puerto Peñasco, W side of Bahía Adair, 14.III.2010, B. T. Wilder et al. 45 (ARIZ, UCR, USON). Municipio Sonoyta, Río Sonoyta, $0.5 \mathrm{~km}$ S of Cerro El Huérfano, 23 airline km WNW from Sonoyta, 25.X.1992, R. S. Felger et al. 977 (MEXU); Río Sonoyta, $21 \mathrm{~km}$ on Mex 2, W of Sonoyta at 1 $\mathrm{km} \mathrm{W}$ of Quitobaquito and $1 \mathrm{~km} \mathrm{~S}$ of highway, 4.X.1985, $R$. S. Felger y K. Van Houten 971 (MEXU); 21.35 km (línea recta) al SE del poblado de Golfo de Santa Clara, 24.III.2011, J. J. Sánchez 133 (USON). 
Pluchea yucatanensis G.L. Nesom, Phytologia 67(2): 160. 1989. TIPO: MÉXICO. Campeche, In savannah, Champoton, 7.VII.1932, W. C. Steere 1844 (holotipo: LL00373735!, isotipo: US-00288790!).

Hierbas perennes, 0.4-0.6 m de alto; tallos sin alas; ramas glandulares, en ocasiones glabrescentes con la edad; hojas sésiles, láminas oblongo-elípticas, $3.1-5 \mathrm{~cm}$ de largo, 1.3-2.5 cm de ancho, ápice agudo, base auriculada, margen serrado, glandulares en ambas superficies; inflorescencias corimbiformes con 8-20 cabezuelas, pedúnculos individuales $0.4-4.5 \mathrm{~mm}$ de largo, glandulares; involucro campanulado 5.4-5.8 mm de largo, (5.2-)6-6.5 $\mathrm{mm}$ de ancho; filarios 24-28, 3-4 seriados, imbricados, elípticos, ápice agudo, glandulares; flores periféricas 188 200 , corolas 3.6-4 mm de largo; cipselas $0.74-1 \mathrm{~mm}$ de largo, pardas, pilosas y glandulares; vilano con $28-30$ cerdas capilares, 4-5 mm de largo; flores del disco 26-30, corolas 4.5-5.3 mm de largo, rosadas, lóbulos lanceolados, glandulares en el ápice; anteras 1.2-1.5 mm de largo; estilo 5-6.3 $\mathrm{mm}$ de largo; cipselas 1-1.2 mm de largo, pardas o negras, pilosas y glandulares; vilano con 22-25 cerdas capilares, 5-6.2 $\mathrm{mm}$ de largo.

Comentarios taxonómicos: Pluchea yucatanensis presenta similitudes morfológicas con $P$. mexicana y $P$. baccharis. Sin embargo, se diferencia de ellas porque carece de glándulas en las hojas, tiene pedúnculos individuales glandulares, mayor número de flores periféricas ( $\geq 188 \mathrm{vs}$. $\leq 130)$.

Distribución: en México, Pluchea yucatanensis se encuentra en Campeche y Quintana Roo, en elevaciones de 0-20 m s.n.m. (Fig. 7).

Hábitat: sabanas y vegetación hidrófila; en suelos arenosos, a orillas de cuerpos de agua.

Fenología: florece y fructifica en julio.

Ejemplares examinados: MÉXICO. Quintana Roo, municipio Felipe Carrillo Puerto, Laguna de Chonyaxche, $20 \mathrm{~km}$ de Tulum hacia el SO, 22.VII.1979, A. Novelo y A. Lot 848
(MEXU). Municipio José María Morelos, Lake Chichancanab, 28.VII.1932, W. C. Steere 2417 (F).

Pseudoconyza Cuatrec., Ciencia (Mexico) 21(1): 30. 1961. TIPO: Pseudoconyza lyrata (Kunth) Cuatrec.

Hierbas perennes, aromáticas; tallos sin alas, pilosos; hojas alternas, sésiles o cortamente pecioladas, pinnatisectas, ovadas, elípticas o elíptico-ovadas; inflorescencias cimosas o corimbiformes; involucro campanulado; filarios en 3-4 series, graduadas, receptáculo convexo, glabro, desnudo; flores periféricas numerosas, femeninas, corolas filiformes, rosadas; flores del disco (2-)12-20, hermafroditas, corolas tubulares, rosadas o blanquecinas; anteras blanquecinas, apéndices apicales triangulares o lanceolados, base caudada; ramas del estilo redondeadas en el ápice, con papilas obtusas; cipselas elipsoides, rojizas, estriadas, pilosas; vilano con cerdas capilares lisas.

Comentarios taxonómicos: Pseudoconyza es un género monotípico que se distribuye en trópicos y subtrópicos de América, Asia y África (Pruski, 2018). Las relaciones filogenéticas del género aún no están resueltas, pues forma parte de una politomia con Coleocoma $\mathrm{F}$. Muell., Cylindrocline, Doellia, Epaltes, Karelinia, Laggera, Peuchel-loeschea O. Hoffm., Pluchea, Porphyrostemma Grant ex Benth. \& Hook. f., Sphaeranthus L., Streptoglossa Steetze ex F. Muell. y Tessaria Ruiz \& Pav., que en conjunto reciben el nombre de núcleo Plucheoide (Anderberg, 2009).

Pseudoconyza viscosa (Mill.) D’Arcy, Phytologia 25(5): 281. 1973.

इConyza viscosa Mill., Gard. Dict. (ed. 8). Conyza no. 8. 1768. TIPO: MÉXICO. Veracruz, Localidad no especificada, 1730, W. Houston s.n. (holotipo: BM-001009462!, foto E-00531323!).

इ Conyza lyrata Kunth, Nov. Gen. Sp. (folio ed.) 4: 55. 1820. TIPO: ECUADOR. Guayaquil, Crescit in humidis prope Guayaquil Quitensium, II.1799, F. W. H. A. Humboldt y A. J. A. Bonpland 3812 (holotipo: P-00322294!, isotipos: HAL0112793!, P-01816029!). 
इ Erigeron lyratus (Kunth) M. Gómez, Anales Soc. Esp. Hist. Nat. 19(2): 272. 1890.

E Eschenbachia lyrata (Kunth) Britton \& Millsp., Bahama Fl. 444. 1920.

= Conyza chiapensis Brandegee, Univ. Calif. Publ. Bot. 10(8): 419. 1924. TIPO: MÉXICO. Chiapas, Jalisco, IV.1923, C. A. Purpus 8987 (holotipo: UC-220280!, isotipos: GH00022891! MO-193515!, NY-00167638!, UC-220281!, US00129245!).

$\equiv$ Blumea lyrata (Kunth) V.M. Badillo, Bol. Soc. Venez. Ci. Nat., 10(67): 257. 1946.

三 Pseudoconyza lyrata (Kunth) Cuatrec., Ciencia (Mexico) 21(1): 31. 1961.

三 Laggera lyrata (Kunth) Leins, Mitt. Bot. Staatssamml. München 9: 107. 1971.

इ Blumea viscosa (Mill.) V.M. Badillo, Revista Fac. Agron. (Maracay) 7(3): 9. 1974.

इ Blumea viscosa var. lyrata (Kunth) D’Arcy, Phytologia 30(1): 5. 1975.

Hierbas perennes, 0.15-2 m de alto; ramas tomentulosas, piloso-glandulares; hojas con peciolos cortos, 1.2-10 $\mathrm{mm}$ de largo; lámina elíptica u ovado-elíptica, $1.9-6.2 \mathrm{~cm}$ de largo, 0.7-2.9 cm de ancho, ápice agudo a veces obtuso, base cuneada, rara vez decurrente, margen dentado, piloso-glandulares en ambas superficies; inflorescencias con 6-200 cabezuelas, pedúnculos individuales $1-10 \mathrm{~mm}$ de largo, piloso-glandulares; involucro campanulado 5.9-10 $\mathrm{mm}$ de largo, 5.2-13.8 mm de ancho; filarios 36-50, 4-5 seriados, imbricados, lanceolados o linear-lanceolados, ápice agudo, piloso-glandulares; flores periféricas 120-150(-182), corolas 3.8-5.2 mm de largo; flores del disco 12-20, corolas 2.6-6.4 mm de largo, cipselas 0.5-0.8 mm de largo, rojizas, pilosas; vilano con 10-12 cerdas capilares, 3.2-4.6 mm de largo, rosadas, a veces blanquecinas, lóbulos oblongoovados, papilosos en el ápice; anteras 1.3-2.5 mm de largo; estilo 2.7-5.6 mm de largo; cipselas $0.5-1.2 \mathrm{~mm}$ de largo, rojizas, pardas cuando inmaduras, pilosas; vilano con 10-14 cerdas capilares, 2.6-5 mm de largo (Fig. 9).

Comentarios taxonómicos: la ubicación taxonómica de $P$. viscosa ha variado considerablemente a través del tiempo. La especie tipo Conyza viscosa y C. lyrata, uno de sus sinónimos, se incluyeron en la tribu Asteraceae (MiIler, 1768; Cuatrecasas, 1961). No obstante, una revisión minuciosa de las características florales permitió reubicarla en la tribu Inuleae, considerando las anteras caudadas, indumento en la superficie dorsal de las ramas del estilo, ausencia de apéndices deltoideos en las anteras y cipselas ellipsoides (D’Arcy, 1973). Badillo (1974) transfirió P. viscosa a Blumea DC., un género del viejo mundo, que tendría a $B$. viscosa como su único representante en América (Dillon y Sagástegui Alva, 1991). Sin embargo, en un trabajo reciente, Pruski (2018) considera que la ubicación taxonómica de $P$. viscosa en Blumea fue producto de la similitud morfológica existente en los caracteres vegetativos de algunas especies de Blumea y $P$. viscosa. Aunque los caracteres florales la diferencian claramente de Blumea, entre ellos, la presencia de papilas en la región distal del estilo (vs. estilo glabro), ramas del estilo con papilas obtusas (vs. papilas agudas) y cipselas rojizas, estriadas (vs. cipselas pardas, costilladas).

Distribución: en México, Pseudoconyza viscosa se encuentra en Campeche, Chiapas, Colima, Guerrero, Jalisco, Michoacán, Morelos, Nayarit, Oaxaca, Puebla, Sinaloa, Sonora, Veracruz y Yucatán, en elevaciones de 0-1750 m s.n.m. (Fig. 10).

Hábitat: encinares, manglares, matorrales xerófilos, selvas altas perennifolias, bajas caducifolias, vegetación de dunas costeras, halófila, hidrófila y secundaria. En suelos aluviales, arenosos, arcillosos, salinos, ocasionalmente pedregosos o derivados de rocas volcánicas.

Fenología: florece y fructifica de noviembre a julio.

Ejemplares examinados: MÉXICO. Campeche, municipio Calkini, $4 \mathrm{~km}$ al $\mathrm{O}$ de Tunkasche, sobre el camino Calkini - Punta Arenas, 25.III.1988, E. Cabrera y H. de Cabrera 15844 (MEXU). Municipio Hopelchén, Xpujil-Zoh laguna, 6.IV.1986, E. Góngora 244 (MEXU). Chiapas, municipio Ocozocoautla de Espinosa, steep-walled canyon at the head of the Río de la Venta at the Chorreadero near Derna, 20. III.1973, D. E. Breedlove 34307 (MEXU, MO). Municipio San Fernando, dirt road between San Fernando and Maravillas, near Lago Malpaso, 4-66 mi NW of San Fernando, 15. 


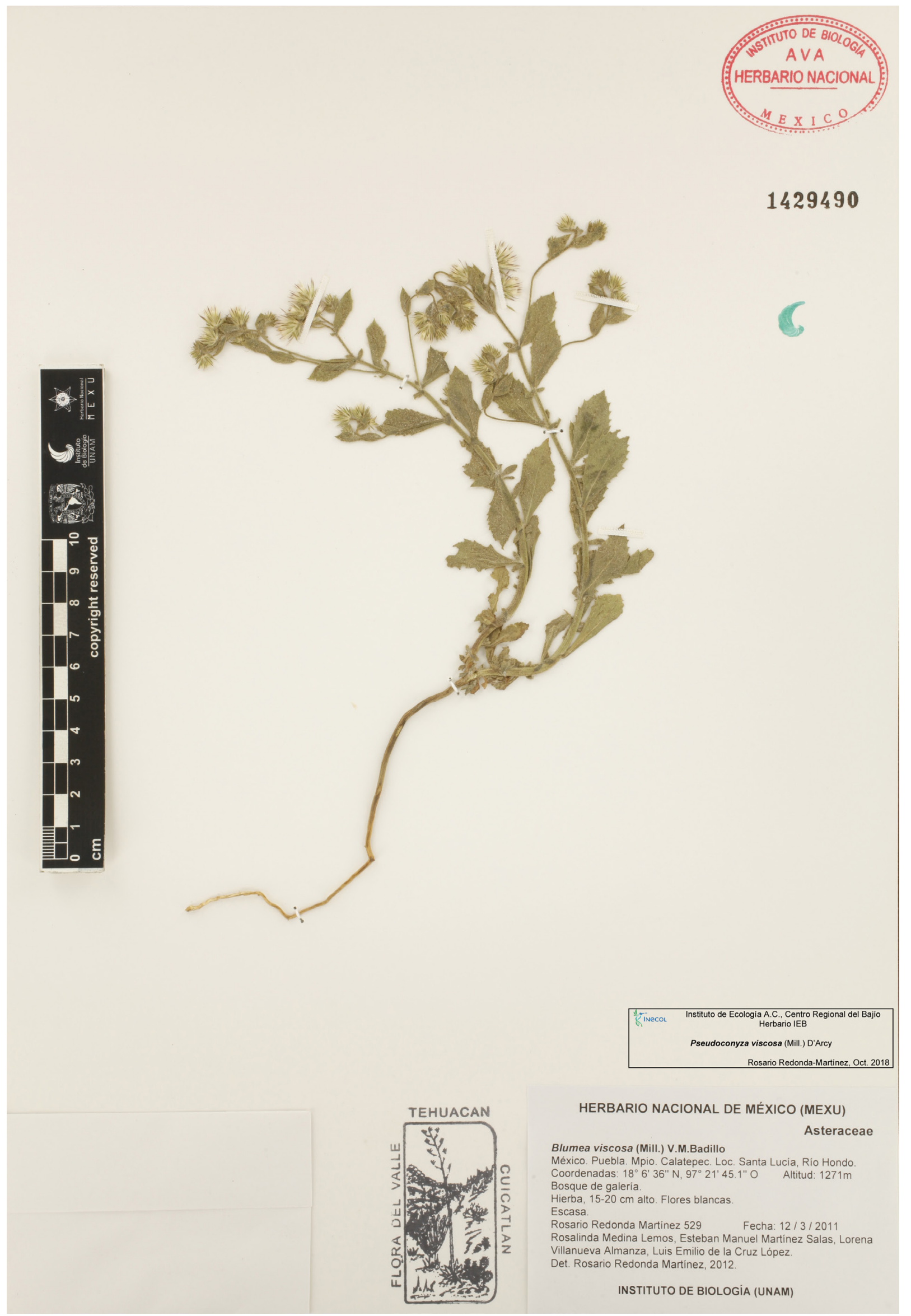

Figura 9: Ejemplar herborizado de Pseudoconyza viscosa (Mill.) D’Arcy. 


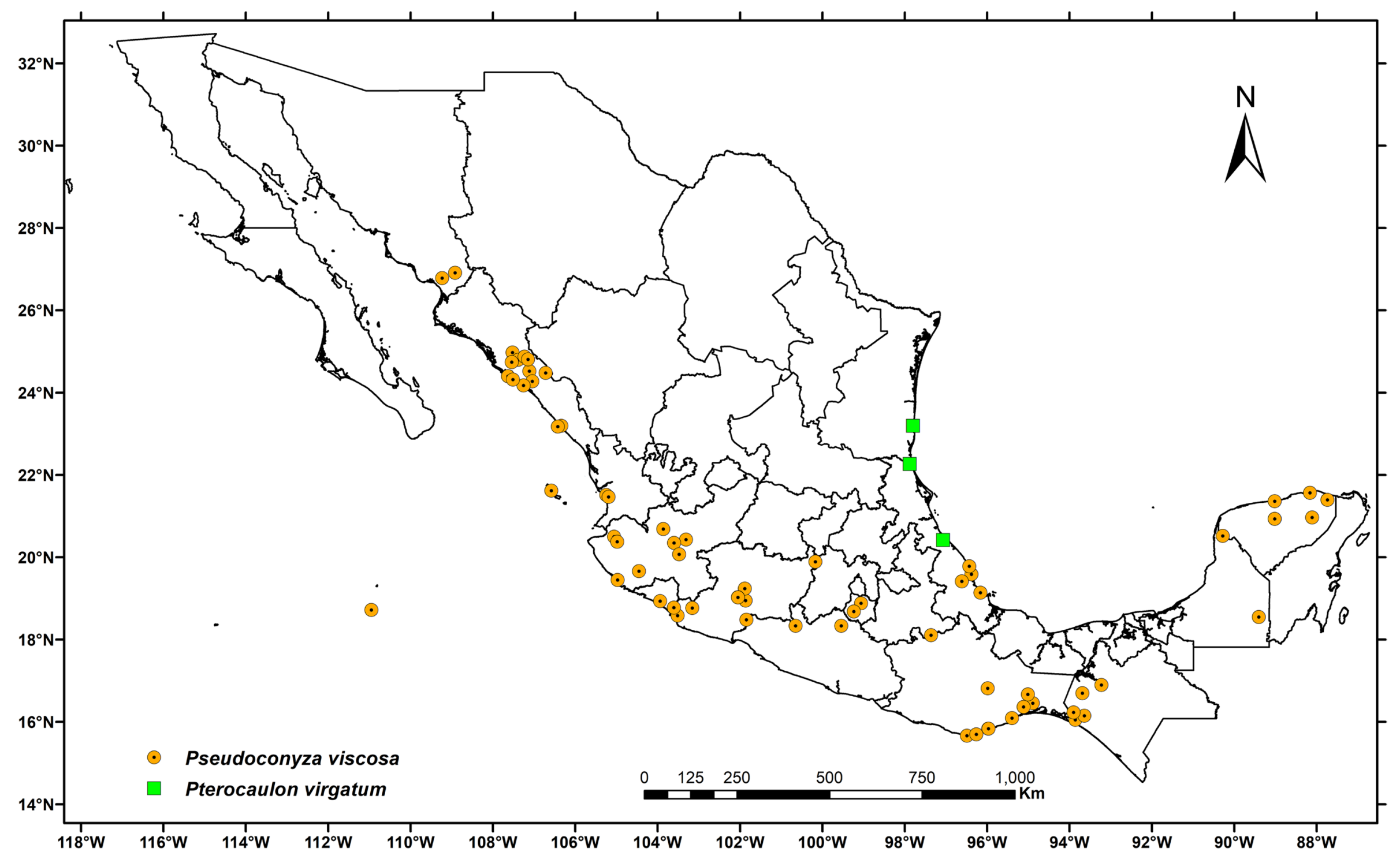

Figura 10: Mapa de distribución geográfica en México de Pseudoconyza viscosa (Mill.) D’Arcy y Pterocaulon virgatum (L.) DC. 
II.1987, T. B. Croat y D. P. Hannon 65037 (MEXU, MO). Municipio Tonalá, Paredón, 20.I.1946, E. Matuda 16358 (MEXU, MO); ejido Costa Rica, Reserva de la Biosfera La Sepultura, 16.III.2002, E. Mélendez et al. 33 (MEXU). Colima, municipio Manzanillo, $\mathrm{N}$ of Bahía, Socorro Island, 16. IV.1987, G. A. Levin 1767 (MEXU). Municipio Tecomán, Ruíz Cortínez, 21.III.1985, J. Maillet 3 (MEXU). Guerrero, municipio Coahuayutla de Guerrero, El Maguey, $3.45 \mathrm{~km}$ al N, 20.III.1999, J. Calónico y R. Mayorga 14321 (FCME, MEXU). Municipio Iguala de la Independencia, región zona $\mathrm{N}$ del estado, 17.II.1987, A. Almazán 130 (MEXU). Municipio Pungarabato, Pungarabato, distrito Coyuca, 1.XI.1934, G. B. Hinton et al. 5451 (MEXU, MO). Jalisco, municipio Acatlán de Juárez, margen E Laguna de Atotonilco, 19.IV.1987, L. M. Villarreal 13119 (MEXU). Municipio Casimiro Castillo, highway 15-18 road miles SW of Autlán, 9.IV.1951, $R$. McVaugh 11960 (MEXU). Municipio La Huerta, Arroyo Tapeixtes, La Mina, km 55 de la carretera Barra de Navidad Puerto Vallarta, $4 \mathrm{~km}$ al SE de la Estación Biológica, 27.V.1985, E. J. Lott 2521 (MEXU); Rancho Cuixmala, Cumbres 1 Arroyo Cajones, 14.I.1991, E. J. Lott et al. 3264 (MEXU, MO); Chamela by region, Rancho Cuixmala, Río Cuixmala, $0.5 \mathrm{mi}$ downriver from headquarters Bahía Chamela, 11.V.1991, E. J. Lott et al. 3377 (MEXU, MO). Municipio Teocuitatlán de Corona, camino Poncitlán - Tehuantepec, 15.V.1993, E. Villegas y M. A. Macías 285 (MEXU). Municipio Teuchitlán, nacimiento del Río Ameca, 4. IV.1976, L. M. Villarreal 8468 (MEXU). Municipio Tlajomulco de Zúñiga, Orilla de la laguna, en los bungalows, Cajititlán, 21.IV.1998, C. Cortés 492 (MEXU). Michoacán, municipio Aquila, $3 \mathrm{~km}$ al SO de Aquila, 30.III.1981, J. C. Soto et al. 2857 (MEXU). Municipio Coahuayana, Los Tanques, 2-3 $\mathrm{km}$ al $\mathrm{N}$ de Camalote (Achotán), por el camino a Palos Marías, 27.X.2010, E. Carranza e I. Silva 7549 (IEB). Municipio Coalcomán de Vázquez Pallares, Llano Coalcomán, 17. III.1939, G. B. Hinton y J. C. Hinton 13624 (ARIZ, MO). Municipio Copándaro, cerca del balneario La Ciénega, próximo a San Agustín del Maíz, 3.V.1986, J. Rzedowski 39654 (IEB, MEXU). Municipio La Huacana, Río Huámito, Ichamio, 30.V.2003, S. Rangel 660 (IEB, MEXU). Municipio Múgica, Río El Marqués, $200 \mathrm{~m}$ al SE del puente sobre la autopista Morelia - Lázaro Cárdenas, 1.II.2004, V. W. Steinmann y J. M. Porter 4054 (IEB); 5.5 km al NE de la salida a Nueva Italia, por la autopista Lázaro Cárdenas Morelia, 1.V.2006, V. W. Steinmann y Y. Ramírez 5392 (IEB). Municipio Nuevo Urecho, $15 \mathrm{~km}$ S of Terán by the Morelia - Lázaro Cárdenas autopista, arroyo below the highway, 26.I.2002, V. W. Steinmann y E. Carranza 2290 (IEB, MEXU). Morelos, municipio Puente de Ixtla, Xoxocotla a $50 \mathrm{~m}$ del entronque calle Constituyentes Atlaquiahuac, 22.II.1986, S. A. Ortiz y N. Romero 110 (MEXU); vereda de Avenida Constituyentes y 20 de noviembre Xoxocotla, 22.II.1986, S. A. Ortiz y N. Romero 976 (MEXU). Municipio Yautepec, waste places Yautepec, 22.V.1904, C. G. Pringle 8986 (MEXU, MO, NY). Nayarit, municipio Bahía de Banderas, Club de golf Flamingos, cerca de Bucerías, 18.III.2005, M. A. Cházaro y R. Romero 8469 (IEB). Municipio Compostela, Playa Platanitos, 29.VI.2007, D. Jimeno et al. 392 (IEB). Municipio San Blas, Playas de Los Cocos, s.f., J. González 81 (MEXU); Isla María Madre, 15.V.1925, O. Solís s.n. (MEXU); Las Islitas a 5 $\mathrm{km}$ al SE de San Blas, camino a Miramar, 20.VI.1987, O. Téllez y J. Miller 10500 (MEXU, MO, NY). Municipio Santiago Ixcuintla, Isla Isabel, 16.XII.1977, F. Ramos 20 (MEXU). Oaxaca, municipio Asunción Ixtaltepec, Camino al paraje Agua Tibia, $1 \mathrm{~km}$ al $\mathrm{N}$ de Nizanda, 15.VII.2000, E. A. Pérez y B. Reyes 1885 (MEXU). Municipio Juchitán de Zaragoza, 14.2 $\mathrm{km}$ al E, 78 ${ }^{\circ}$, del centro de Juchitán, 9.IV.2006, A. Saynes 6221 (MEXU). Municipio Oaxaca, Oaxaca, 16.IV.1900, C. R. Orcutt 3187 (MO). Municipio Salina Cruz, El Cangrejo, 2 km al S de Morro Mazatlán, 6.II.1987, C. Martínez 848 (MEXU). Municipio San Antonio Nanahuatipan, $2 \mathrm{~km}$ al $\mathrm{N}$ de Casa Blanca, 17.IV.1987, A. Salinas y C. H. Ramos F-3894 (IEB). Municipio San Blas Atempa, $12.7 \mathrm{~km}$ al E, 69 $9^{\circ}$ del centro de San Blas Atempa, distrito Tehuantepec, 19.II.2006, A. Saynes y R. de Salas 6205 (MEXU). Municipio San Pedro Pochutla, Puerto Ángel, 25.XII.1974, W. L. Boege 3315 (MEXU). Municipio Santa María Huatulco, a un lado del Río Guajinicuil, sobre la brecha a la Laguna Culebra, 27.IV.2006, A. Sánchez et al.1425 (IEB). Municipio Santiago Astata, Barra de la Cruz, Laguna del Potrerón, 2.IV.1998, M. Elorsa 121 (MEXU); Zimatán, $2 \mathrm{~km}$ S del puente, rancho Boca del Río, 5.II.1999, M. Elorsa y D. Scheidegger 1658 (MEXU); $1 \mathrm{~km}$ al SE de Barra de La Cruz, camino a La Playa, 16.V.1994, E. Martínez S. et al. 32179 (MEXU). Puebla, municipio Caltepec, Santa Lucía, Río Hondo, 12.III.2011, R. Redonda et al. 529 (MEXU). Municipio Coxcatlán, 8 km al SE de Coxcatlán, 
sobre la carretera a Teotitlán del Camino, 31.III.1968, J. Rzedowski 25590 (ENCB, IEB). Sinaloa, municipio Cosalá, 12 km al NO de Cosalá, 5.IV.1986, E. L. Carrasco et al. 98 (MEXU). Municipio Culiacán, Salvador Alvarado, 24.Il.1988, G. A Bojórquez y H. Aguilar 452 (MEXU); cerros alrededor de Imala, 4.IV.1995, G. A. Bojórquez et al. 1620 (MEXU); Sindicatura de El Salado, alrededores de El Álamo, 3.V.1995, G. A. Bojórquez et al. 1657 (MEXU); Península de Lucenilla, 10.IV.1988, F. Hernández et al. 701 (MEXU); Sindicatura de Higueras de Abuya, $3.5 \mathrm{~km}$ al $\mathrm{N}$ de Higueras Abuya, 6. VI.1995, F. Hernández et al. 1381 (MEXU); Península de Villamoros, 4 km de entrada a la Península, 27.IV.1995, J. A. Hernández et al. 386 (MEXU); Península de Lucenilla, 20 km de la entrada de la península, 24.V.1985, F. Hernández y J. A. Gutiérrez 153 (MEXU); cerro La Chiva, km 22 carretera Culiacán - Guamuchil al NE del Limón de los Ramos, 24. III.1984, J.A. Prado y V. Llamas 62 (MEXU); Culiacán, 7. III.1988, R. Vega y I. F. Vega 2660 (MEXU). Municipio Mazatlán, El Castillo, Sindicatura Mazatlán, III.1931, J. González 6919 (MEXU); Isla Redonda frente al Puerto de Mazatlán, 18.VI.1977, L. M. Villarreal 10464 (MEXU). Municipio Navolato, carretera 50 a $3 \mathrm{~km}$ de Campo Gobierno rumbo a San Pedro, 22.IV.1988, G. A. Bojórquez y H. Aguilar 559 (MEXU). Sonora, municipio Álamos, Arroyo El Mentidero at El Chinal road, $11.3 \mathrm{~km}$ S of Alamos, The Río Mayo Region, T. R. Van Devender y S. A. Meyer s.n. (ARIZ); along edge of river; near crossing of Río Cuchujaqui on road to Ranchería; 2.5 mi beyond junction with main road to El Chinal, Sinaloa, and about $13.5 \mathrm{mi}$ S of Alamos, 21.IV.1984, $T$. R. Van Devender et al. s.n. (ARIZ). Municipio Navojoa, Teachive de Masiaca, Arroyo Masiaca, The Río Mayo Region, 18.IV.1984, T. R. Van Devender et al. s.n. (ARIZ). Veracruz, municipio Actopan, Estación de Biología Morro de la Mancha, 13.V.1981, Brigada de dunas 57 (FCME, MEXU). Municipio Alto Lucero de Gutiérrez Barrios, Palma Sola, 6. IV.1972, F. Ventura 5176 (ASU, ENCB). Municipio Emiliano Zapata, El Aguaje, 2.IV.1975, F. Ventura 11160 (ENCB, MEXU); Municipio Veracruz, between Mex. 180 and railroad, ca. 5 mi SW of Veracruz, 15.VI.1971, D. B. Ward 7893 (NY). Yucatán, municipio Calotmul, Pocoboch, s.f., G. F. Gaumer 2406 (MO). Municipio Izamal, Izamal, s.f, G. F. Gaumer 1453 (MO); Izamal, III.1916, G. F. Gaumer 23249 (MO). Municipio Río Lagartos, entronque del camino Río Lagartos a Las Coloradas, 13.III.1985, C. Chan 4835 (MEXU); 1 km al S de Santa Clara, sobre el camino a Dzidzantun, 30.IV.1987, E. Cabrera y H. de Cabrera 13268 (MEXU). Municipio Tizimín, $7.5 \mathrm{~km}$ al E de Dzonot Carretera, $14 \mathrm{~km}$ al $\mathrm{O}$ de la desviación a Dzonot Carretera 15 antes de El Cuyo, por la carretera Colonia Yucatán - El Cuyo, 19.V.2006, J. L. Tapia et al. 1878 (MEXU, MO).

Pterocaulon Elliott, Sketch Bot. S. California 2(4): 323. 1824. TIPO: Pterocaulon pycnostachyum (Michx.) Elliott

= Monenteles Labill., Sert. Austro-Caledon. 42, t. 43. 1825.

= Chlaenobolus Cass., Dict. Sci. Nat. (ed. 2) 49: 337. 1827.

= Pluchea subg. Chlaenobolus Cass., Dict. Sci. Nat. (ed. 2) 49: 337. 1827.

Hierbas perennes o arbustos; tallos alados, lanosos, tomentosos o piloso-glandulares; hojas alternas, sésiles, lineares o lanceoladas; cabezuelas dispuestas en inflorescencias espiciformes simples o ramificadas, a veces glomérulos; involucro campanulado, ocasionalmente hemisférico, filarios 3-4 seriados, graduados, receptáculo plano o cóncavo, glabro o piloso, desnudo; flores periféricas numerosas, femeninas, corolas filiformes, rosadas o blanquecinas; flores del disco una o pocas, hermafroditas o funcionalmente masculinas, corolas infundibuliformes, rosadas; ramas del estilo aplanadas y triangulares, pilosas en el ápice; anteras blanquecinas, ápice ovado-lanceolado; estilo con ramas agudas en el ápice, pilosas; cipselas fusiformes 8-10 costilladas, pardas, pilosas o piloso-glandulares; vilano con cerdas capilares, dispuesto en 1 o 2 series.

Candolle (1836) reconoció dos secciones del género Pterocaulon: Chænobolus con seis especies, y Pteurocaulopsis con una. Ambas secciones se diferencian por el tipo de indumento que presentan en las hojas (tomentoso vs. glabrescente) y el involucro (hirsuto vs. glabrescente). Pterocaulon incluye 18 especies con distribución en América, Asia y Australia; está relacionado con Cratystylis S. Moore, Iphionopsis Anderb., Rhodogeron Griseb. y Sachsia Griseb. (Anderberg, 2009). En México se encuentra únicamente Pterocaulon virgatum (L.) DC. 
Pterocaulon virgatum (L.) DC., Prodr. 5: 454. 1836.

E Gnaphalium virgatum L. TIPO: JAMAICA. Habit in Jamaica, Carolina, s.f., P. Browne s.n. (lectotipo: designado por D'Arcy in Woodson et al., 1975).

三 Conyza virgata (L.) L., Sp. PI. (ed. 2) 2: 1206. 1763.

= Gnaphalium spicatum Mill., Gard. Dict. (ed. 8) Gnaphalium no. 24. 1768. TIPO: JAMAICA. Grows naturally in Jamaica, and in other of the hot parts of America, s.f., Anónimo s.n. (holotipo: no localizado).

= Conyza rugosa Vahl, Symb. Bot. 1: 71. 1790. TIPO: BRASIL. Habitat in Brazilia, s.f., P. Commerson s.n. (holotipo: C-10007802!).

$\equiv$ Chlaenobolus virgata (L.) Cass., Dict. Sci. Nat. (ed. 2) 49: 340. 1827.

इChlaenobolus rugosa (Vahl) Cass., Dict. Sci. Nat. (ed. 2) 49: 341. 1827.

三 Pterocaulon rugosum (Vahl) Malme, Bih. Kongl. Svenska Vetensk.-Akad. Handl. 27(III-12): 16. 1901.

= Pterocaulon pompilianum Standl. \& L.O. Williams, Ceiba 1(2): 94. 1950. TIPO: HONDURAS. Morazán, in boggy savanna near Las Mesas, 2.X.1949, L. O. Williams 16898 (holotipo: US-00129417!).

Hierbas perennes, 0.6-1 m de alto; tallos tomentosos; hojas con lámina linear-lanceolada, $1.9-6.2 \mathrm{~cm}$ de largo, 2.7-8.4 mm de ancho, ápice agudo, base cuneada, decurrente, margen entero, revoluto, haz tomentuloso, envés tomentoso; cabezuelas dispuestas en inflorescencias espiciformes simples, con 80-120 cabezuelas sésiles; involucro campanulado 5.5-6.3 mm de largo, 4.5-5.7 mm de ancho; filarios 52-60, 4-5 seriados, imbricados, lanceolados o linear-lanceolados, ápice agudo, tomentulosos; flores periféricas 62-70, corolas 5-5.4 mm de largo, estilo 4.1-4.3 mm de largo; cipselas 1.4-1.6 mm de largo, pardas, pilosas; vilano uniseriado, con 28-32 cerdas capilares, 5.2$6.2 \mathrm{~mm}$ de largo; flores del disco 2-3, corolas 4.1-4.8 mm de largo, rosadas, lóbulos lanceolados, glandulares en el ápice; anteras 1.6-1.7 mm de largo; estilo 5.02-5.16 mm de largo; cipselas 1.24-1.32 mm de largo, pardas, pilosoglandulares; vilano uniseriado, con $26-30$ cerdas capilares, 5.8-6.4 mm de largo (Fig. 11).
Comentarios taxonómicos: Pterocaulon virgatum se diferencia de otras Plucheinae mexicanas por las inflorescencias espiciformes simples.

Distribución: en México, Pterocaulon virgatum se ha registrado en los estados de Tamaulipas y Veracruz, en elevaciones de 8-25 m s.n.m. (Fig. 10).

Hábitat: se encuentra en encinares, pastizales y vegetación hidrófila.

Fenología: florece y fructifica en agosto.

Ejemplares examinados: MÉXICO. Tamaulipas, municipio Aldama, Rancho Nuevo $23 \mathrm{~km}$ al E del ejido San Rafael, 18.VIII.1984, D. Baro 397 (MEXU). Municipio Tampico, NW side of city of Tampico, 8 m, 17.VIII.1986, M. Nee 32726 (NY). Veracruz, municipio Gutiérrez Zamora, E of Río Tecolutla and S of Hwy Mex 180, 3 km SE of Gutiérrez Zamora, 25 m, 19.VIII.1986, M. Nee 32792 (NY).

\section{Discusión y conclusiones}

En las colecciones científicas aún es común encontrar especímenes de Inuleae erróneamente identificados, ya que las personas que no están familiarizadas con la morfología de las cabezuelas de Asteraceae, llegan a confundirlos con otros grupos, en particular Eupatorieae y Vernonieae, cuya morfología de las cabezuelas aparentemente resulta similar. Aunque a simple vista las cabezuelas de Inuleae son "parecidas" a las de Eupatorieae y Vernonieae porque tienen involucro graduado, flores moradas o rosadas y vilano de cerdas capilares en un número considerable de especies, al observarlas cuidadosamente en el microscopio se aprecian diferencias notables. De esas, la principal es la gran cantidad de flores periféricas con corolas filiformes que, sumada a un número reducido de flores del disco bisexuales o funcionalmente masculinas, permiten distinguir fácilmente un ejemplar de Inuleae de Vernonieae y Eupatorieae. Además, las características de las ramas del estilo difieren de las de Inuleae; en Vernonieae son agudas y pilosas en toda su extensión en ambas superficies, mientras que en Eupatorieae son clavadas y glabras (Funk et al., 2009). 


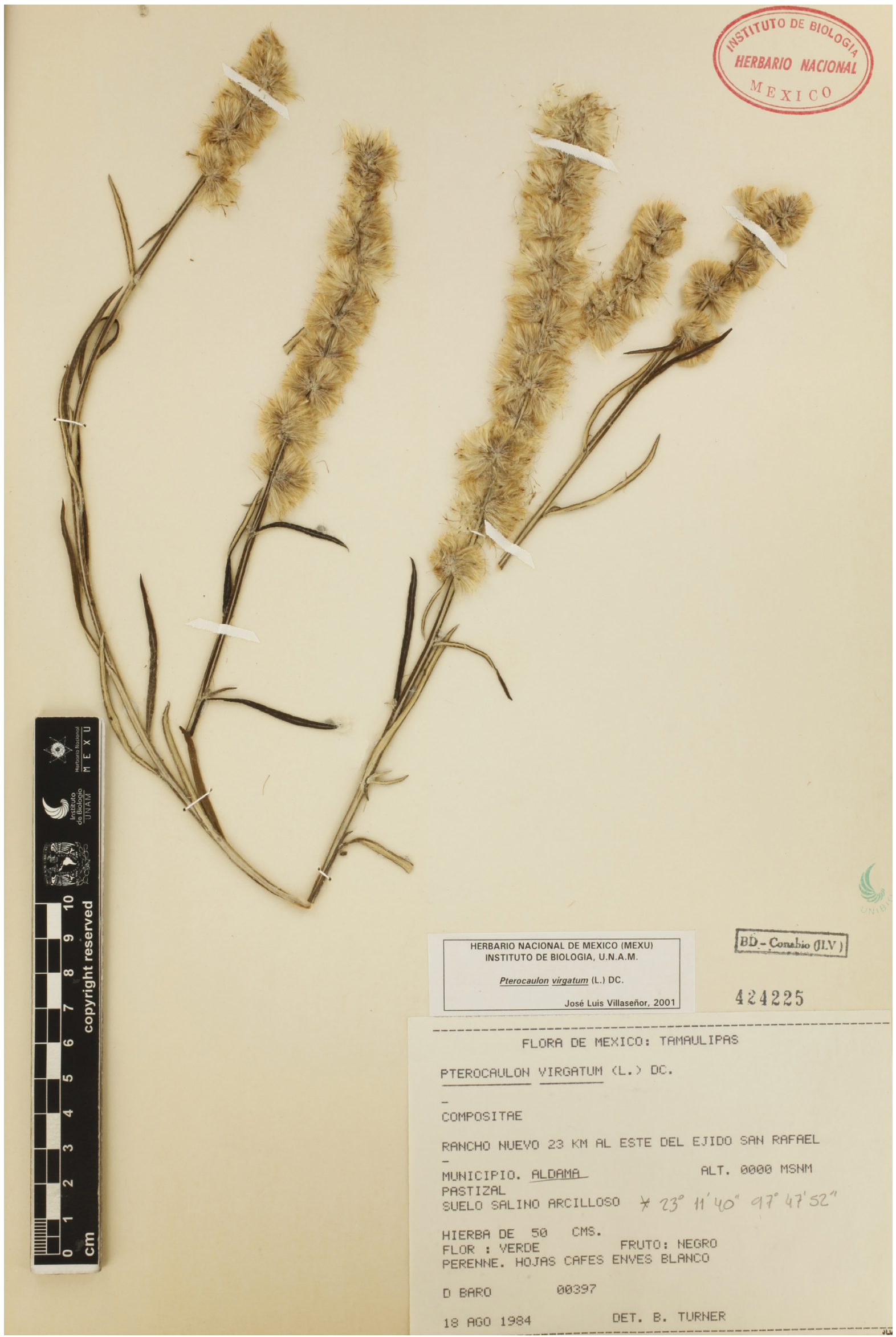

Figura 11: Ejemplar herborizado de Pterocaulon virgatum (L.) DC. 
En cuanto al número de especies, en el presente estudio se encontraron similitudes con los datos presentados en trabajos florísticos regionales (McVaugh, 1984; Medina-Lemos y Villaseñor-Ríos, 2010; Pruski, 2018; Redonda-Martínez, 2016); se reconoce nuevamente la validez de Pseudoconyza, también hubo diferencias en el número de especies y variedades de Pluchea, género que cuenta con un trabajo taxonómico de las especies mexicanas (Villaseñor y Villarreal, 2006), en el que se citó la presencia de $P$. foetida. Sin embargo, ahora se sabe que se trata de una identificación incorrecta de $P$. baccharis (Pruski, 2005), ya que ambos taxones presentan similitudes morfológicas considerables, pero difieren en el arreglo de las cabezuelas en la inflorescencia (laxas vs. densas), color de filarios y flores (rojizas o moradas vs. blanquecinas o amarillentas), e indumento de los filarios (víscido aracnoideo vs. aracnoideo y glandular) (Pruski, 2005; Nesom, 2020).

Aunque a simple vista las diferencias parecen mínimas y se prestan para una identificación errónea, en especial porque la coloración de algunas estructuras suele perderse con el proceso de secado de los ejemplares botánicos, la variación en el agrupamiento de cabezuelas y el indumento de los filarios han demostrado ser caracteres potencialmente útiles para distinguir especies cercanamente relacionadas en Inuelae (McVaugh, 1984; Pruski, 2005, 2018) y otros grupos de Asteraceae (Funk et al., 2009). Sin embargo, es necesario realizar estudios anatómicos y micromorfológicos de las especies mexicanas agrupadas en el complejo P. foetida para corroborar su separación. En Asteraceae la micromorfología y anatomía son importantes herramientas taxonómicas para diferenciar taxones (King y Robinson, 1970; Janaćković et al., 2019), tanto en grupos basales (Erbar, 2016; Panero y Funk, 2002, 2008; Roque y Funk, 2013) como recientes (King y Robinson, 1970; Funk et al., 2009, Schilling et al., 2013). Aunque en México existen pocos trabajos al respecto, estos también han sido efectivos para reconocer subtribus (Redonda-Martínez, 2017, 2018b; Redonda-Martínez et al., 2017), géneros (RedondaMartínez y Villaseñor, 2014; García-Mendoza et al., 2020) y delimitar especies (Redonda-Martínez y Martínez Salas, 2015, 2019; Redonda-Martínez et al., 2016, 2020; Redonda-
Martínez y Mora-Jarvio, 2017; Redonda-Martínez, 2018a; Villaseñor y Redonda-Martínez, 2018).

\section{Contribución de autores}

RMM planteó el estudio, revisó el material en colecciones científicas y digitales, elaboró el escrito y las figuras, revisó y aprobó la versión final del trabajo.

\section{Financiamiento}

Este trabajo se financió con recursos propios.

\section{Agradecimientos}

Se agradece a los curadores de los herbarios ENCB, FCME, IEB y MEXU por las facilidades otorgadas para consultar las colecciones. También a Ricardo Rivera y Maribel Paniagua Ibáñez por la ayuda brindada en la captura de información de algunas colecciones científicas. María del Rosario García Peña (MEXU) escaneó los ejemplares que ilustran este trabajo. Rafael Colorado apoyó en la búsqueda de algunas referencias bibliográficas. Finalmente, se agradecen los comentarios y sugerencias de dos revisores anónimos y del equipo editorial, para mejorar la versión final del manuscrito.

\section{Literatura citada}

Anderberg, A. A. 1989. Phylogeny and reclassification of the tribe Inuleae (Asteraceae). Canadian Journal of Botany 67: 2277 2296. DOI: https://doi.org/10.1139/b89-292

Anderberg, A. A. 2009. Inuleae. In: Funk, V. A., A. Sussana, T. F. Stuessy y R. J. Bayer (eds.). Systematics, Evolution and Biogeography of the Compositae. International Association for Plant Taxonomy. Viena, Austria. Pp. 667-680.

Anderberg, A. A. y P. Eldenäs. 2007. Tribe Inuleae. In: Kadereit, J. W. y C. Jeffrey (eds.). The families and Genera of Vascular Plants. Flowering Plants. Eudicots. Asterales, Vol. VIII. Springer. Berlin, Germany. Pp. 374-391.

Anderberg, A. A., P. Eldenäs, R. J. Bayer y M. Englund. 2005. Evolutionary relationships in the Asteraceae tribe Inuleae (incl. Plucheeae) evidenced by DNA sequences of $n d h F$; with notes on systematic positions of some aberrant genera. Organisms, Diversity \& Evolution 5(2): 135-146. DOI: https://doi.org/10.1016/j.ode.2004.10.015 
Badillo, V. M. 1974. Blumea viscosa y Piptocarpa cuatrecasiana dos nuevas combinaciones en Compositae. Revista de la Facultad de Agronomía 7(3): 9-16.

BCFM. 2018. Botanical Collections. The Field Museum. Chicago, USA. $\quad$ https://collections-botany.fieldmuseum.org/list (consultado julio de 2018).

BCS. 2018. Botany Collection Search, Smithsonian National Museum of Natural History. Washington D.C., USA. http:// collections.nmnh.si.edu/search/botany/?v=s1\#new-search (consultado julio de 2018).

Bentham, G. 1873. Ordo LXXXVIII: Compositae. In: Bentham, G. y J. D. Hooker (eds.). Genera Plantarum, Vol. 2. Reeve and Co. London, UK. Pp. 163-533.

Blake, S. F. 1930. Notes on certain type specimens of American Asteraceae in European herbaria. Contributions from the United States National Herbarium 25: 227-263.

Blake, S. F. 1931. Nine new American Asteraceae. Journal of the Washington Academy of Sciences 21: 325-336.

Britten, J. 1898. The Conyzas of Miller's dictionary (ed. 8). Journal of Botany, British and Foreing 36: 51-55.

Candolle, A. P. de 1836. Prodromus Systematis Regni Vegetabilis, Vol. 5. Treutel et Würtz. Paris, Gallia. 706 pp.

Cassini, A. H. G. 1817. Aperçu des genres nouveaux formés par $M$. Henri Cassini dans la famille des Synanthérées. Troisiéme Fascicule. Bulletin des Sciences, par la Société Philomatique 1817(11): 31-34.

Cassini, A. H. G. 1818a. Aperçu des genres nouveaux formés par M. Henri Cassini dans la famille des Synanthérées. Huitiéme Fascicule. Bulletin des Sciences, par la Société Philomatique 1818: 71-77.

Cassini, A. H. G. 1818b. Aperçu des genres nouveaux formés par M. Henri Cassini dans la famille des Synanthérées. Neuviéme Fascicule. Bulletin des Sciences, par la Société Philomatique 1818: 139-142.

Cassini, A. H. G. 1819. Suite du Sixiéme Mémoire sur la famille des Synanthérées, contenant les caractères des tribus. Journal de Physique, de Chimie,d'Historie Naturelle et des Arts 88: 189-204.

CICY. 2010+. Flora de la Península de Yucatán. Centro de Investigación Científica de Yucatán, Mérida, México. http://www.cicy.mx/ sitios/flora\%20digital (consultado junio de 2019).
Cuatrecasas, J. 1961. Notas sobre Astereas andinas. Ciencia (Mexico) 21: 21-32.

D’Arcy, W. G. 1973. A name change in Pseudoconyza (CompositaeInuleae). Phytologia 25(5): 281.

De-Nova, J. A., P. Castillo-Lara, A. K. Gudiño-Cano y J. GarcíaPérez. 2018. Flora endémica del estado de San Luis Potosí y regiones adyacentes en México. Árido-Ciencia 3(1): 21-41.

Dillon, M. A. y A. Sagástegui Alva. 1991. Family Asteraceae: Part $\mathrm{V}$, Tribe Inuleae. Flora of Peru. Fieldiana Botany, New Series 26: 1-70.

Eldenäs, P., M. Källersjö y A. A. Anderberg. 1999. Phylogenetic placement and circumscription of tribes Inuleae s.str. and Plucheeae (Asteraceae): evidenced from sequences of chloroplast gene ndhF. Molecular Phylogenetics and Evolution 13(1): 50-58. DOI: https://doi.org/10.1006/ mpev.1999.0635

Erbar, C. 2016. Unique style morphology in the monotypic Famantinanthoideae-Famantinantheae, a recently established subfamily and tribe of Asteraceae. Systematic Botany 41(3): 796-806. DOI: https://doi. org/10.1600/036364416X692415

ESRI. 2013. ArcMap 10.2. Environmental Systems Research Institute. Rendlands, USA.

Flora de Veracruz. 1978+. Flora de Veracruz. Instituto de Ecología, A.C. Xalapa, México. http://www1.inecol.edu.mx/floraver/ (consultado junio de 2019).

Funk, V. A., A. Sussana, T. F. Stuessy y H. Robinson. 2009. Classification of Compositae. In: Funk, V. A., A. Sussana, T. F. Stuessy y R. J. Bayer (eds.). Systematics, Evolution and Biogeography of the Compositae. International Association for Plant Taxonomy. Viena, Austria. Pp. 171-189.

García-Mendoza, A. J., D. Sandoval-Gutiérrez y R. RedondaMartínez. 2020. Mixtecalia, a new monotypic genus of the subtribe Tussilagininae (Senecioneae, Asteraceae) from the state of Oaxaca, Mexico. Phytotaxa 438(2): 119-132. DOI: https://doi.org/10.11646/phytotaxa.438.2.5

Gillis, W. T. 1977. Pluchea revisited. Taxon 26(5/6): 581-591. DOI: https://doi.org/10.2307/1219662

Goodfrey, R. K. 1952. Pluchea, section Stylimnus, in North America. Journal of the Elisha Mitchell Scientific Society 68(2): 238-275. 
Google Earth. 2018. Google Earth Pro version 7.3.2.5491., Mountain View, USA. https://www.google.com/earth/ download/ge/ (consultado julio de 2018).

Gray, A. 1862. Enumeration of a collection of dried plants made by L.J. Xantus, at Cape San Lucas, in Lower California, between august, 1859, and february, 1860, and communicated to the Smithsonian Institution. Proceedings of the American Academy of Arts and Sciences 5: 153-173.

Holland, M. M. y P. G. Risser. 1991. The role of landscape boundaries in the management and restoration of changing environments: introduction. In: Holland, M. M. (ed.). Ecotones. The role of landscape boundaries in the management and restoration of changing environments. Chapman \& Hall. New York, USA. Pp. 1-7. DOI: https://doi. org/10.1007/978-1-4615-9686-8_1

INEGI. 2017. Guía para interpretación de información cartográfica impresa y digital del Carta de uso de suelo y vegetación serie IV. Instituto Nacional de Estadística y Geografía. Aguascalientes, México. 204 pp.

Janaćković, P., A. Sussana y P. D. Marin. 2019. Micromorphology and anatomy in systematics of Asteraceae. An old-fashioned approach? Biologica Nyssana 10(2): 77-85. DOI: https://doi. org/10.5281/zenodo.3600177

JSTOR. 2018. Global Plants on Jstor. http://plants.jstor.org/ (consultado mayo de 2018).

Khan, R. y C. E. Jarvis. 1989. The correct name for the plant known as Pluchea symphytifolia (Miller) Gillis (Asteraceae). Taxon 38(4): 659-662. DOI: https://doi.org/10.2307/1222661

Kim, K.-J. y R. K. Jansen. 1995. ndhF sequence evolution and the major clades in the sunflower family. Proceedings of the National Academy of Sciences of the United States of America 92(22): 10379-10383. DOI: https://doi. org/10.1073/pnas.92.22.10379

King, R. M. y H. Robinson. 1970. The New Synantherology. Taxon 19(1): 6-11. DOI: https://doi.org/10.2307/1217907

Mandel, J. R., R. B. Dikow, C. M. Siniscalchi, R. Thapa, L. E. Watson y V. A. Funk. 2019. A fully resolved backbone phylogeny reveals numerous dispersals and explosive diversifications throughout the history of Asteraceae. Proceedings of the National Academy of Sciences 116(28): 14083-14088. DOI: https://doi.org/10.1073/pnas.1903871116
McVaugh, R. 1984. Compositae. In: Anderson, W. R. (ed.). Flora Novo-Galiciana 12. The University of Michigan Press. Ann Arbor, USA. Pp. 1-1157.

Medina-Lemos, R. y J. L. Villaseñor-Ríos. 2010. Asteraceae, Tribu Plucheeae. In: Medina-Lemos, R., J. G. Sánchez-Ken, A. García-Mendoza y S. Arias-Montes (eds.). Flora del Valle de Tehuacán-Cuicatlán. Instituto de Biología, Universidad Nacional Autónoma de México. México, D.F., México. 78: 1-13.

Merxmüller, H., P. Leins y H. Roessler. 1977. Inuleae. Systematic review. In: Heywood, V. H., J. B. Harborne y B. L. Turner (eds.). The biology and chemistry of the Compositae, Vol 1. Academic Press. London, UK. Pp. 577-602.

Miller, P. 1768. The gardeners dictionary. 8th ed. Miller. London, UK. Miranda, F. y E. Hernández X. 1963. Los tipos de vegetación de México y su clasificación. Boletín de la Sociedad Botánica de México. 28: 29-179. DOI: https://doi.org/10.17129/ botsci.1084

Nelson, A. 1924. Eremohylema. University of Wyoming Publications ins Science. Botany 1(3): 54.

Nesom, G. L. 1989. New Species, New Sections, and a taxonomic overview of American Pluchea (Compositae: Inuleae). Phytologia 67(2): 158-167. DOI: https://doi.org/10.5962/bhl. part. 26148

Nesom, G. L. 2004. Notes on typification in Pluchea (Asteraceae: Plucheae). Sida 21: 59-64.

Nesom, G. L. 2020. Pluchea. In: Flora of North America Editorial Committee (eds.). 1993+ Flora of North America North of Mexico, Vols. 19-21. New York and Oxford, USA and UK. pp. 478.

NYBG. 2018. The New York Botanical Garden Virtual Herbarium. New York, USA. http://sweetgum.nybg.org/science/vh/ (consultado agosto de 2018).

Panero, J. L. y V. A. Funk. 2002. Toward a phylogenetic subfamilial classification for the Compositae (Asteraceae). Proceedings of the Biological Society of Washington 115(4): 909-922.

Panero, J. L. y V. A. Funk. 2008. The value of sampling anomalous taxa in phylogenetic studies: Major clades of the Asteraceae revealed. Molecular Phylogenetics and Evolution 47: 757782. DOI: https://doi.org/10.1016/j.ympev.2008.02.011

Panero, J. L. y B. S. Crozier. 2016. Macroevolutionary dynamics in the early diversification of Asteraceae. Molecular 
Phylogenetics and Evolution 99: 116-132. DOI: https://doi. org/10.1016/j.ympev.2016.03.007

Pruski, J. F. 2005. Studies of Noetropical Compositae-I. Novelties in Calea, Clibadium, Conyza, Llerasia and Pluchea. Sida 21(4): 2023-2037.

Pruski, J. F. 2018. Asteraceae. XIII. Tribu Inuleae Cass. In: Davidse, G., M. Sousa S., S. Knapp y F. Chiang (eds.). Flora Mesoamericana 5.2. Missouri Botanical Garden Press. St. Louis, USA. Pp. 346-353.

Rabinowitz, S., S. Cairns y T. Dillon. 1986. Seven forms of rarity and their frequency in the flora of British Isles. In: Soule, M. E. (ed.). Conservation Biology: The Science of Scarcity and Diversity. Sinauer Associates Inc. Sunderland, USA. Pp. 182-203.

Redonda-Martínez, R. 2016. Compositae, Tribu Inuleae. Flora del Bajío y de Regiones Adyacentes 194: 1-13.

Redonda-Martínez, R. 2017. Morfología floral de la subtribu Leiboldinae (Vernonieae, Asteraceae). Brittonia 69(4): 504515. DOI: https://doi.org/10.1007/s12228-017-9487-z

Redonda-Martínez, R. 2018a. Tratamiento taxonómico de la tribu Mutisieae (Asteraceae) en México. Acta Botanica Mexicana 123: 121-166. DOI: https://doi.org/10.21829/ abm123.2018.1277

Redonda-Martínez, R. 2018b. Taxonomic revisión of subtribe Leiboldiinae (Vernonieae, Asteraceae). Systematic Botany 43(1): 344-363. DOI: https://doi. org/10.1600/036364418X696996

Redonda-Martínez, R. y J. L. Villaseñor. 2014. Stramentopappus congestiflorus (Asteraceae: Vernonieae: Leiboldiinae) a new species from Oaxaca, Mexico. Revista Mexicana de Biodiversidad 85(1): 1-8. DOI: https://doi.org/10.7550/ rmb.36354

Redonda-Martínez, R. y E. M. Martínez Salas. 2015. Lepidonia alba (Asteraceae: Vernonieae: Leiboldiinae) a new species from the state of Chiapas, Mexico. Systematic Botany 40(4): 11371143. DOI: https://doi.org/10.1600/036364415X690157

Redonda-Martínez, R. y E. M. Martínez Salas. 2019. A new species of Oxylobus (Eupatorieae, Asteraceae) and updated taxonomic treatment of the genus. Systematic Botany 44(3): 719-735. DOI: https://doi.org/10.1600/03636441 9X1560114943873
Redonda-Martínez, R. y M. Mora-Jarvio. 2017. Vernonia occulta (Asteraceae: Vernonieae: Vernoniinae) a new species from the state of Oaxaca, Mexico. Systematic Botany 42(2): 378383. DOI: https://doi.org/10.1600/036364417X695493

Redonda-Martínez, R., J. L. Villaseñor y A. Campos-Villanueva. 2016. A new species of Vernonia (Asteraceae, Vernonieae) from the state of Oaxaca, Mexico. Phytotaxa 282(3): 211217. DOI: https://doi.org/10.11646/phytotaxa.282.3.4

Redonda-Martínez, R., T. Terrazas, y A. Rojas-Leal. 2020. Morphoanatomy of cypselae of native species of Mutisieae (Asteraceae) from Mexico. Phytotaxa 436(1): 1-20. DOI: https://doi.org/10.11646/phytotaxa.436.1.1

Redonda-Martínez, R., T. Terrazas, A. Rojas-Leal y J. L. Villaseñor. 2017. The morpho-anatomy of cypselae in subtribe Leiboldiinae (Vernonieae, Asteraceae). Brazilian Journal of Botany 40(2): 491-502. DOI: https://doi.org/10.1007/ s40415-016-0349-9

Roque, N. y V. A. Funk. 2013. Morphological characters add support for some members of the basal grade of Asteraceae. Botanical Journal of the Linnean Society 171(3): 568-586. DOI: https://doi.org/10.1111/boj.12000

Schilling, E. E., J. L. Panero, B. S. Corzier y P. Dávila Aranda. 2013. Relationships of Asanthus (Asteraceae, Eupatorieae). Systematic Botany 38(1): 253-258. DOI: https://doi. org/10.1600/03634413X661999

SEINet. 2018. Southwest Enviromental Information Network (SEINet) Arizona-New Mexico Chapter. http://swbiodiversity. org/seinet/collections/harvestparams.php (consultado septiembre de 2018).

Thiers, B. 2019. Index Herbariorum. A global directory of public herbaria and associated staff. New York Botanical Garden's Virtual Herbarium. New York, USA. http://sweetgum.nybg. org/science/ih/ (consultado marzo de 2019).

TROPICOS. 2018. Tropicos.org. Missouri Botanical Garden. Missouri, USA. http://www.tropicos.org (consultado octubre de 2018).

Villaseñor, J. L. 2018. Diversidad y distribución de la familia Asteraceae en México. Botanical Sciences 96(2): 332-358. DOI: https://doi.org/10.17129/botsci.1872

Villaseñor, J. L. y J. A. Villarreal. 2006. El género Pluchea (familia Asteraceae, tribu Plucheeae) en México. Revista 
Mexicana de Biodiversidad 77(1): 59-65. DOI: https://doi. org/10.22201/ib.20078706e.2006.001.318

Villaseñor, J. L. y R. Redonda-Martínez. 2018. A new species of Dahlia (Asteraceae, Coreopsideae) from the state of Oaxaca, Mexico. Phytotaxa 362(2): 239-243. DOI: https:// doi.org/10.11646/phytotaxa.362.2.11
Woodson, R. E., R. W. Schery, W. G. D’Arcy, T. S. Elias, P. Busey, R. M. King, H. Robinson, T. F. Stuessy, J. M. Canne, D. J. Keil, T. M. Barkley, R. C. Gardner, B. B. Simpson y A. S. Tomb. 1975. Flora of Panama, Part IX-Family 184. Compositae. Annals of the Missouri Botanical Garden 62(4): 835-1321. DOI: https://doi.org/10.2307/2395231 